\title{
CHECKLIST OF HINTON'S COLLECTIONS OF THE FLORA OF SOUTH-CENTRAL NUEVO LEON AND ADJACENT COAHUILA
}

JAMES HinTON

AND

GEORGE S. Hinton

Rancho Aguililla, Galeana, Nuevo León

Apdo. Postal 603, Saltillo, Coahuila, México 25000

\begin{abstract}
A preliminary checklist of the flora of south-central Nuevo León and part of adjacent Coahuila includes 1955 species, in 713 genera and 130 families. At least 200 species, an estimated 10 percent of the total flora, are endemic to this area, mostly on exposed gypsum and on mountain peaks at the highest elevations. The region of investigation includes the municipios of Allende, Aramberri, Dr. Arroyo, Galeana, Iturbide, Linares, Montemorelos, Rayones, Sabinas Hidalgo, Salinas Victoria, Santa Catarina, Santiago, Villa Aldama, Villa de García and Zaragoza in Nuevo León and Arteaga and Ramos Arizpe in Coahuila, and includes habitats ranging in elevation from 475 to 3700 meters.
\end{abstract}

\section{RESUMEN}

Se presenta una lista preliminar de la flora del sur y centro de Nuevo León y parte adyacente de Coahuila, la cual incluye 1955 especies en 713 géneros y 130 familias. Por lo menos 200 especies, aproximadamente 10 por ciento de la flora total, son endémicas a esta área, principalmente en suelos yesosos y en cimas de montañas de mayor elevación. La región de investigación abarca los municipios de Allende, Aramberri, Dr. Arroyo, Galeana, Iturbide, Linares, Montemorelos, Rayones, Sabinas Hidalgo, Salinas Victoria, Santa Catarina, Santiago, Villa de Aldama, Villa de García y Zaragoza en Nuevo León, Arteaga y General Cepeda en Coahuila, en un intervalo altitudinal entre 475 y 3700 m.

The number of species of vascular plants in México is estimated to be about 22,800 (Rzedowski, 1993). The accuracy of this estimate, however, remains to be tested, because published accounts (as regional floras or checklists) of the plants of México are few. This is particularly true of northern México. A flora of the Chihuahuan Desert Region, however, encompassing most of Coahuila and adjacent areas of Chihuahua, Durango, Zacatecas, and San Luis Potosí is nearing publication (Johnston and Henrickson, in prep.) and preparation of a floristic account of Tamaulipas is underway (Hernández and Martínez, in prep.). Rzedowski (1978) has provided general descriptions of the vegetation of this area.

From the rich area of sierran Nuevo León and Coahuila in northeastern México, there are summaries of the flora of the peak of Cerro Potosí (Beaman and Andresen, 1966; García- 
Arévalo and González-Elizondo, 1991) and alpine areas very limited in size (McDonald, 1990), but there has been no attempt to provide a detailed characterization of the flora of this whole area. We have made collections of vascular plants in about 344 localities within the municipios of Allende, Aramberri, Dr. Arroyo, Galeana, Iturbide, Linares, Montemorelos, Rayones, Sabinas Hidalgo, Salinas Victoria, Santa Catarina, Santiago, Villa Aldama, Villa de García and Zaragoza in Nuevo León and Arteaga and Ramos Arizpe in Coahuila. The localities are plotted in figure 1 of the Appendix, each square representing from a few to several hundred collections. An enumeration of our 6550 collections from this area is reported in list 3 of the Appendix, which we regard as preliminary to a broader and more detailed treatment of the flora of the whole state of Nuevo León. We are aware that other taxa have been collected from this area, but we believe that our collections cover about $75 \%$ of the species in the flora. More than 1955 species are represented in our collections (some remain unidentified or undescribed) belonging to 713 genera and 130 families, a summary of which is found in list 2 of the Appendix. Family names are alphabetically arranged, as well as the generic names and species epithets. At least 200 species (ca. 10 percent of the total) apparently are endemic to this area. Over 80 new taxa have been described from our collections alone, mostly within the past 5 years, and are reported in list 1 of the Appendix. Also, we have collected a significant number of species previously unknown to Nuevo León or Coahuila, and several unknown to México.

The area covered in our studies ranges from 475-3700 meters in elevation. At the lower elevations, the vegetation is characteristic of the Chihuahuan Desert, with Larrea, Yucca, Agave, Opuntia, Gymnosperma, etc.; above this is found matorral (Yucca and Agave with other shrubs) at ca. 1100-1800 m, juniper and oak-juniper woodlands at ca. 1800-2800 $\mathrm{m}$, oak and pine woodlands at ca. 1800-3200 m, and pine-fir and alpine meadows at the highest elevations. Cactaceae are particularly abundant and characteristic of the flora up to about 2000 meters.

Endemic species are found primarily in two areas: the highest mountain peaks (ca. 2800-3700 meters) and exposed gypsum (ca. 1000-2200 meters). These two habitats tend to have sharply reduced numbers of species, but those that do occur there are usually very distinctive in morphology and have highly restricted geographic ranges. The distinctive nature of the gypsum in northeastern México has been discussed by Turner and Powell (1979).

The initial identifications of our collections (ca. 1969-1979) were made by J. Rzedowski; voucher specimens for these are deposited at ENCB and in the Herbarium of G. B. Hinton. The majority of the identifications since 1980 have been made by B. L. Turner and G. Nesom; a set of vouchers for these is deposited at LL, TEX, ENCB, MEXU and the Herbarium of G. B. Hinton. Authorities in various taxonomic groups have also contributed identifications: L. Constance (Apiaceae), L. Hernández (Agavaceae), M. Mayfield (Euphorbiaceae), A. McDonald (Convolvulaceae), M. Martínez (Solanaceae), T. Patterson (Pinaceae), T. P. Ramamoorthy (Lamiaceae), J. and C. Reeder (Poaceae), C. Todzia (Orchidaceae), S. Zamudio (Pinguicula), D. Hunt (Commelinaceae), A. LeDuc (Nyctaginaceae) and A. Hempel (Loasaceae).

The collection numbers of plants from northeastern México are an extension of the series begun by Geo. B. Hinton in 1931 (see Hinton and Rzedowski, 1975). We (James Hinton and George S. Hinton, representing the second and third generations of botanical collectors in our family) have collected to a large degree separately but we have also collected together, a fact that is not indicated on the collection labels or the numbers. 
Hinton \& Hinton: Hintons' Collections from South-Central Nuevo León and Adjacent Coahuila

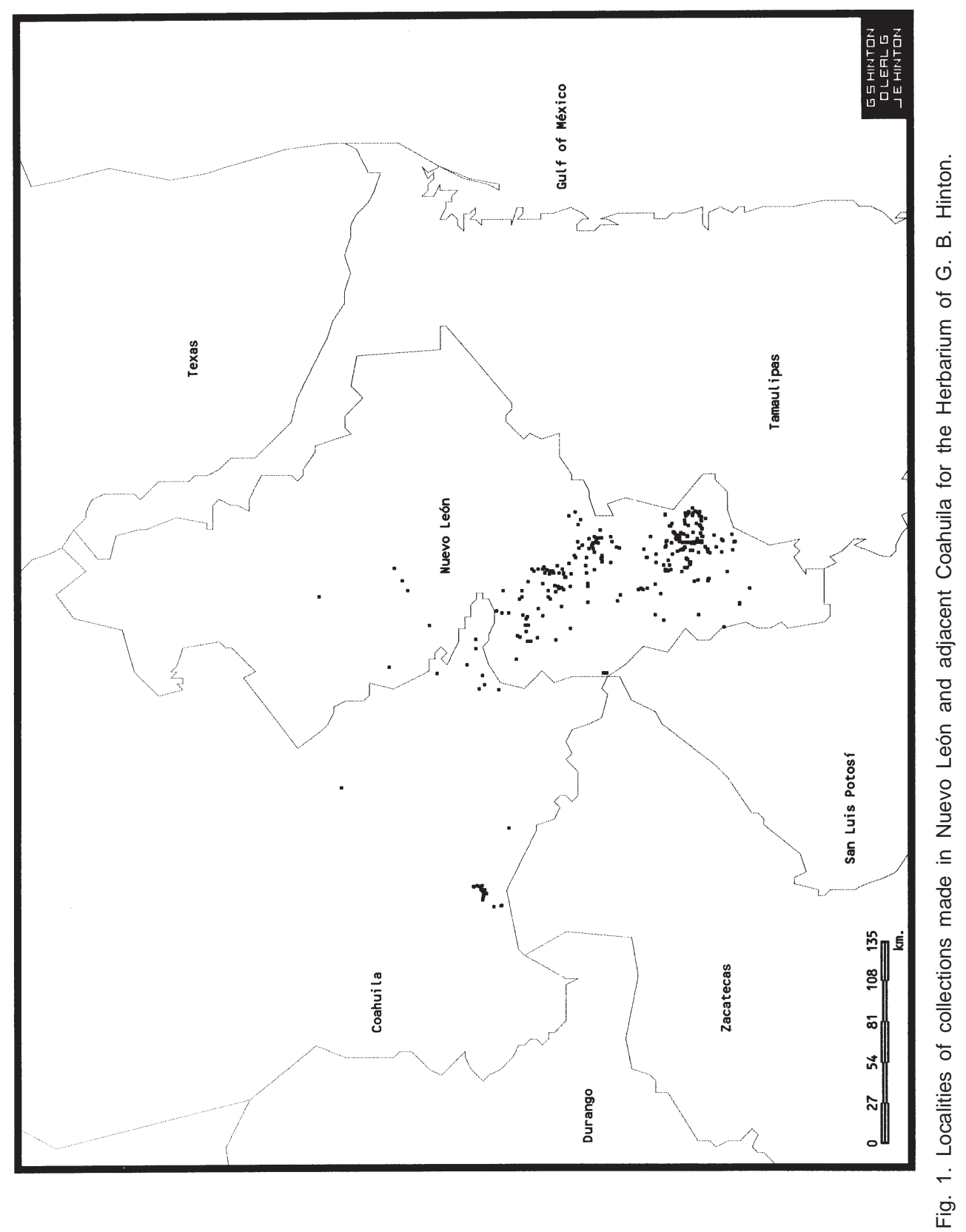


We wish to express herewith our deep appreciation for the help and encouragement received in our endeavor from Billie L. Turner and Guy Nesom; and to Guy Nesom in particular for his help in the preparation of this paper. Without their help and inspiration we would probably be doing something else.

We also thank an unknown reviewer for his suggestions to this manuscript.

\section{LITERATURE CITED}

Beaman, J. H. and J. W. Andresen. 1966. The vegetation, floristics and phytogeography of the summit of Cerro Potosi, México. Amer. Midl. Natur. 75: 1-33.

García-Arévalo, A. and S. González-Elizondo. 1991. Flora y vegetación de la cima del Cerro Potosí, Nuevo León, México. Acta Bot. Mex. 13: 53-74.

Hinton, J. and J. Rzedowski. 1975. George B. Hinton, explorador botánico en el sudoeste de México. Anales Esc. Nac. Cienc. Biol. 21: 1-114.

McDonald, A. J. 1990. The alpine-subalpine flora of northeastern México. Sida 14: 21-28.

Rzedowski, J. 1978. Vegetación de México. Editorial Limusa, S. A. México, D. F. 432 pp.

Rzedowski, J. 1993. Biological diversity of México: origins and distribution. In: Ramamoorthy, T. P., R. Bye, A. Lot and J. Fa, eds. Oxford University Press. New York. pp. 129-144.

Turner, B. L. and A. M. Powell. 1979. Deserts, gypsum and endemism. In: Goodin, J. R. and D. K. Northington (eds.). Arid land plant resources. International Center for Arid and Semi-arid Land Studies. Texas Tech University, Lubbock, Texas. pp. 96-116. 
Hinton \& Hinton: Hintons' Collections from South-Central Nuevo León and Adjacent Coahuila

List 1. Partial list of new species from Nuevo León and adjacent Coahuila in the Herbarium of $\mathrm{G}$. B Hinton.

\begin{tabular}{|c|c|c|}
\hline NUMBER & FAMILY & NAME \\
\hline 20354 & ACANTHACEAE & Justicia hintoniorum Nesom \\
\hline 21186 & AGAVACEAE & Hemiphylacus hintoniorum L. Hernández (ined.) \\
\hline 22924 & AGAVACEAE & Manfreda sp. nov. B. L. Turner \\
\hline 18410 & APIACEAE & Arracacia hintonii Const. \& Affolter \\
\hline 18426 & APIACEAE & Tauschia hintoniorum Const. \& Affolter \\
\hline 17935 & ASCLEPIADACEAE & $\begin{array}{c}\text { Cynanchum mccartii var. latifolium B. L. Turner ex } \\
\text { Henrickson }\end{array}$ \\
\hline 23934 & ASTERACEAE & Acourtia hintoniorum B. L. Turner \\
\hline 18779 & ASTERACEAE & Ageratina gypsophila B. L. Turner \\
\hline 20837 & ASTERACEAE & Ageratina ilicifolia B. L. Turner \\
\hline 22484 & ASTERACEAE & Ageratina viejoana B. L. Turner \\
\hline 20499 & ASTERACEAE & Ageratina zapalinama B. L. Turner \\
\hline 23980 & ASTERACEAE & Ageratina sp. nov. B. L. Turner (\#1) \\
\hline 23811 & ASTERACEAE & Ageratina sp. nov. B. L. Turner (\#2) \\
\hline 20050 & ASTERACEAE & Brickellia aramberrana B. L. Turner \\
\hline 17970 & ASTERACEAE & Brickellia hintoniorum B. L. Turner \\
\hline 18868 & ASTERACEAE & Erigeron hintoniorum Nesom \\
\hline 21512 & ASTERACEAE & Eupatorium galeanum B. L. Turner \\
\hline 20037 & ASTERACEAE & Eupatorium hintoniorum B. L. Turner \\
\hline 18902 & ASTERACEAE & Gnaphalium flavocephalum Nesom \\
\hline 17263 & ASTERACEAE & Gnaphalium hintoniorum Nesom \\
\hline 18666 & ASTERACEAE & Grindelia hintoniorum Nesom \\
\hline 20486 & ASTERACEAE & Guterrezia grandis Blake (ined.) \\
\hline 18876 & ASTERACEAE & Hymenopappus hintoniorum B. L. Turner \\
\hline 20788 & ASTERACEAE & Perymenium hintoniorum B. L. Turner \\
\hline 17048 & ASTERACEAE & Senecio hintoniorum B. L. Turner \\
\hline 23969 & ASTERACEAE & Senecio viejoanus B. L. Turner (ined.) \\
\hline 18763 & ASTERACEAE & Solidago ericamerioides Nesom \\
\hline 17264 & ASTERACEAE & Solidago hintoniorum Nesom \\
\hline 18333 & ASTERACEAE & Solidago orientalis (Nesom) Nesom \\
\hline 21611 & ASTERACEAE & Stevia hintoniorum B. L. Turner \\
\hline 22031 & ASTERACEAE & Tridax hintoniorum B. L. Turner \\
\hline 22359 & ASTERACEAE & Verbesina aramberrana B. L. Turner \\
\hline 18211 & BORAGINACEAE & Antiphytum hintoniorum Higgins \& B. L. Turner \\
\hline 21950 & BORAGINACEAE & Lithospermum hintoniorum B. L. Turner (ined.) \\
\hline 21528 & CACTACEAE & Aztekium hintonii Glass \& Fitz. \\
\hline 21527 & CACTACEAE & Geohintonia mexicana Glass \& Fitz. \\
\hline 21805 & CACTACEAE & Turbinicarpus sp. nov. Glass \& Fitz. (\#1) \\
\hline 24010 & CACTACEAE & Turbinicarpus sp. nov. Glass \& Fitz. (\#2) \\
\hline 23706 & CARYOPHYLLACEAE & Arenaria gypsostrata B. L. Turner \\
\hline 20957 & CARYOPHYLLACEAE & Arenaria hintoniorum B. L. Turner \\
\hline 17108 & CARYOPHYLLACEAE & Stellaria hintoniorum B. L. Turner \\
\hline 23112 & COMMELINACEAE & Callisia hintoniorum B. L. Turner \\
\hline 22082 & COMMELINACEAE & Gibasis gypsophila B. L. Turner \\
\hline 23013 & COMMELINACEAE & Gibasis hintoniorum B. L. Turner \\
\hline
\end{tabular}


List 1. Continuation.

$\begin{array}{ll}\text { NUMBER } & \text { FAMILY } \\ 24830 & \text { EUPHORBIACEAE } \\ 22118 & \text { FABACEAE } \\ 22936 & \text { FABACEAE } \\ 24053 & \text { FABACEAE } \\ 22354 & \text { GENTIANACEAE } \\ 20564 & \text { HYDROPHYLLACEAE } \\ 18223 & \text { HYDROPHYLLACEAE } \\ 18453 & \text { HYDROPHYLLACEAE } \\ 17868 & \text { HYDROPHYLLACEAE } \\ 22440 & \text { IRIDACEAE } \\ 23737 & \text { IRIDACEAE } \\ 20531 & \text { LAMIACEAE } \\ 19259 & \text { LAMIACEAE } \\ 21276 & \text { LAMIACEAE } \\ 21348 & \text { LAMIACEAE } \\ 23059 & \text { LAMIACEAE } \\ 18999 & \text { LAMIACEAE } \\ 18213 & \text { LAMIACEAE } \\ 18294 & \text { LAMIACEAE } \\ 17545 & \text { LAMIACEAE } \\ 23508 & \text { LAMIACEAE } \\ 22661 & \text { LENTIBULARIACEAE } \\ 24000 & \text { LENTIBULARIACEAE } \\ 21444 & \text { LILIACEAE } \\ 17200 & \text { LILIACEAE } \\ 19679 & \text { OLEACEAE } \\ 22636 & \text { ORCHIDACEAE } \\ 23259 & \text { ORCHIDACEAE } \\ 21284 & \text { ORCHIDACEAE } \\ 21876 & \text { PAPAVERACEAE } \\ 17243 & \text { POACEAE } \\ 18765 & \text { POLYPODIACEAE } \\ 18191 & \text { ROSACEAE } \\ 18422 & \text { SCROPHULARIACEAE } \\ 22321 & \text { SCROPHULARIACEAE } \\ 20929 & \text { SCROPHULARIACEAE } \\ 19166 & \text { SCROPHULARIACEAE } \\ 21009 & \text { SCROPHULARIACEAE } \\ 22104 & \text { SOLANACEAE } \\ & \end{array}$

NAME

Poinsettia tubadenia Mayfield (ined.) Lotus hintoniorum B. L. Turner Lupinus hintoniorum B. L. Turner Sophora juanhintoniana B. L. Turner Geniostemon gypsophilum B. L. Turner Nama hintoniorum Nesom Phacelia hintoniorum B. L. Turner Phacelia potosina B. L. Turner Phacelia zaragozana B. L. Turner Sisyrinchium hintoniorum Nesom Sisyrinchium microbracteatum Nesom Salvia sp. nov. Ramamoorthy (\#1) Salvia sp. nov. Ramamoorthy (\#2) Salvia sp. nov. Ramamoorthy (\#4) Salvia sp. nov. Ramamoorthy (\#5) Satureja hintoniorum B. L. Turner Scutellaria aramberrana B. L. Turner Scutellaria hintoniorum Henrickson Stachys hintoniorum B. L. Turner Stachys langmaniae Rzed. \& Rzed. Stachys sp. nov. B. L. Turner Pinguicula hintoniorum B. L. Turner Pinguicula jorgehintonii B. L. Turner Allium hintoniorum B. L. Turner Zigadenus hintoniorum B. L. Turner Menodora hintoniorum B. L. Turner Galeottiella hintoniorum Todzia (ined.) Malaxis chica Todzia (ined.)

Malaxis hintonii Todzia Hunnemannia hintoniorum Nesom Festuca hintoniana E. Alexeev Cheilanthes hintoniorum Mend. \& Nesom Cercocarpus mexicanus Henrickson Castilleja galehintoniae Nesom Leucophyllum alejandrae Nesom Leucophyllum hintoniorum Nesom Penstemon galloensis Nesom Penstemon luteus Nesom Physalis sp. nov. Martinez (\#1) 
Hinton \& Hinton: Hintons' Collections from South-Central Nuevo León and Adjacent Coahuila

List 2. Summary of the collections from Nuevo León and adjacent Coahuila in the Herbarium of G. B. Hinton, with the number of genera, species and ratio of species per genera in each family.

\begin{tabular}{|c|c|c|c|c|c|c|c|}
\hline Family & Gen & $\mathrm{Sp}$ & Sp/Gen & Family & Gen & $\mathrm{Sp}$ & Sp/Gen \\
\hline ACANTHACEAE & 13 & 21 & 1.62 & EUPHORBIACEAE & 14 & 52 & 3.71 \\
\hline ACERACEAE & 1 & 1 & 1 & FABACEAE & 47 & 138 & 2.94 \\
\hline AGAVACEAE & 4 & 12 & 3 & FAGACEAE & 1 & 33 & 33 \\
\hline AMARANTHACEAE & 7 & 12 & 1.71 & FLACOURTIACEAE & 2 & 2 & 1 \\
\hline AMARYLLIDACEAE & 4 & 5 & 1.25 & FRANKENIACEAE & 1 & 1 & 1 \\
\hline ANACARDIACEAE & 4 & 10 & 2.50 & FUMARIACEAE & 1 & 1 & 1 \\
\hline APIACEAE & 12 & 24 & 2 & GARRYACEAE & 1 & 3 & 3 \\
\hline APOCYNACEAE & 4 & 6 & 1.50 & GENTIANACEAE & 8 & 12 & 1.50 \\
\hline AQUIFOLIACEAE & 1 & 1 & 1 & GERANIACEAE & 2 & 5 & 2.50 \\
\hline ARACEAE & 1 & 1 & 1 & GESNERIACEAE & 1 & 1 & 1 \\
\hline ARISTOLOCHIACEAE & 1 & 1 & 1 & HAMAMELIDACEAE & 1 & 1 & 1 \\
\hline ASCLEPIADACEAE & 6 & 24 & 4 & HYDROPHYLLACEAE & 3 & 23 & 7.67 \\
\hline ASTERACEAE & 116 & 424 & 3.66 & HYPERICACEAE & 1 & 2 & 2 \\
\hline BASELLACEAE & 1 & 1 & 1 & IRIDACEAE & 3 & 11 & 3.6 \\
\hline BEGONIACEAE & 1 & 2 & 2 & JUGLANDACEAE & 2 & 6 & 3 \\
\hline BERBERIDACEAE & 1 & 3 & 3 & JUNCACEAE & 1 & 2 & 2 \\
\hline BETULACEAE & 1 & 1 & 1 & KOEBERLINIACEAE & 1 & 1 & 1 \\
\hline BIGNONIACEAE & 2 & 2 & 1 & KRAMERIACEAE & 1 & 4 & 4 \\
\hline BORAGINACEAE & 12 & 36 & 3 & LAMIACEAE & 15 & 90 & 6 \\
\hline BRASSICACEAE & 23 & 41 & 1.78 & LEMNACEAE & 1 & 1 & 1 \\
\hline BROMELIACEAE & 2 & 7 & 3.50 & LENTIBULARIACEAE & 1 & 8 & 8 \\
\hline CACTACEAE & 9 & 19 & 2.11 & LILIACEAE & 13 & 28 & 2.15 \\
\hline CAMPANULACEAE & 2 & 10 & 5 & LINACEAE & 1 & 7 & 7 \\
\hline CAPPARIDACEAE & 1 & 1 & 1 & LOASACEAE & 3 & 8 & 2.67 \\
\hline CAPRIFOLIACEAE & 5 & 7 & 1.40 & LOGANIACEAE & 1 & 5 & 5 \\
\hline CARYOPHYLLACEAE & 6 & 16 & 2.67 & LORANTHACEAE & 2 & 8 & 4 \\
\hline CELASTRACEAE & 4 & 5 & 1.25 & LYTHRACEAE & 3 & 5 & 1.67 \\
\hline CHENOPODIACEAE & 3 & 10 & 3.33 & MALPIGHIACEAE & 6 & 7 & 1.17 \\
\hline CISTACEAE & 1 & 1 & 1 & MALVACEAE & 15 & 29 & 1.93 \\
\hline CLETHRACEAE & 1 & 1 & 1 & MARTYNIACEAE & 2 & 2 & 1 \\
\hline COMMELINACEAE & 5 & 17 & 3.40 & MENISPERMACEAE & 1 & 2 & 2 \\
\hline CONVOLVULACEAE & 5 & 17 & 3.40 & MYRTACEAE & 1 & 1 & 1 \\
\hline CORNACEAE & 1 & 4 & 4 & NYCTAGINACEAE & 6 & 22 & 3.67 \\
\hline CRASSULACEAE & 4 & 13 & 3.25 & OLACACEAE & 1 & 1 & 1 \\
\hline CROSSOSOMATACEAE & $\mathrm{IE} 1$ & 1 & 1 & OLEACEAE & 3 & 8 & 2.67 \\
\hline CUCURBITACEAE & 6 & 9 & 1.50 & ONAGRACEAE & 6 & 22 & 3.67 \\
\hline CUPRESSACEAE & 2 & 8 & 4 & OPHIOGLOSSACEAE & 1 & 1 & 1 \\
\hline CUSCUTACEAE & 1 & 4 & 4 & ORCHIDACEAE & 18 & 35 & 1.94 \\
\hline CYCADACEAE & 1 & 1 & 1 & OROBANCHACEAE & 2 & 4 & 2 \\
\hline CYPERACEAE & 9 & 17 & 1.89 & OXALIDACEAE & 1 & 9 & 9 \\
\hline DIOSCOREACEAE & 1 & 1 & 1 & PALMAE & 1 & 1 & 1 \\
\hline EBENACEAE & 1 & 1 & 1 & PAPAVERACEAE & 3 & 10 & 3.33 \\
\hline EPHEDRACEAE & 1 & 2 & 2 & PASSIFLORACEAE & 1 & 2 & 2 \\
\hline EQUISETACEAE & 1 & 1 & 1 & PHYTOLACCACEAE & 3 & 4 & 1.33 \\
\hline ERICACEAE & 5 & 5 & 1 & PINACEAE & 4 & 17 & 4.25 \\
\hline
\end{tabular}


List 2. Continuation.

\begin{tabular}{|c|c|c|c|c|c|c|c|}
\hline Family & Gen & $\mathrm{Sp}$ & Sp/Gen & Family & Gen & $\mathrm{Sp}$ & $\mathrm{Sp} / \mathrm{Gen}$ \\
\hline PIPERACEAE & 2 & 4 & 2 & SAPINDACEAE & 5 & 7 & 1.40 \\
\hline PLANTAGINACEAE & 1 & 6 & 6 & SAURURACEAE & 1 & 1 & 1 \\
\hline PLATANACEAE & 1 & 3 & 3 & SAXIFRAGACEAE & 5 & 13 & 2.60 \\
\hline PLUMBAGINACEAE & 1 & 2 & 2 & SCHIZAEACEAE & 1 & 1 & 1 \\
\hline POACEAE & 40 & 83 & 2.08 & SCROPHULARIACEAE & E 17 & 44 & 2.59 \\
\hline POLEMONIACEAE & 5 & 12 & 2.40 & SELAGINELLACEAE & 1 & 9 & 9 \\
\hline POLYGALACEAE & 1 & 12 & 12 & SIMAROUBACEAE & 1 & 1 & 1 \\
\hline POLYGONACEAE & 3 & 9 & 3 & SOLANACEAE & 16 & 49 & 3.06 \\
\hline POLYPODIACEAE & 22 & 74 & 3.36 & STERCULIACEAE & 3 & 4 & 1.33 \\
\hline PORTULACACEAE & 3 & 6 & 2 & TAXACEAE & 1 & 1 & 1 \\
\hline PRIMULACEAE & 3 & 5 & 1.67 & TILIACEAE & 2 & 2 & 1 \\
\hline PYROLACEAE & 4 & 5 & 1.25 & TURNERACEAE & 1 & 1 & 1 \\
\hline RANUNCULACEAE & 5 & 10 & 2 & TYPHACEAE & 1 & 1 & 1 \\
\hline RESEDACEAE & 1 & 1 & 1 & ULMACEAE & 1 & 2 & 2 \\
\hline RHAMNACEAE & 6 & 14 & 2.33 & URTICACEAE & 4 & 5 & 1.25 \\
\hline ROSACEAE & 16 & 43 & 2.69 & VALERIANACEAE & 1 & 5 & 5 \\
\hline RUBIACEAE & 8 & 21 & 2.63 & VERBENACEAE & 11 & 33 & 3 \\
\hline RUTACEAE & 7 & 7 & 1 & VIOLACEAE & 2 & 4 & 2 \\
\hline SALICACEAE & 2 & 3 & 1.50 & VITACEAE & 2 & 3 & 1.50 \\
\hline
\end{tabular}


Hinton \& Hinton: Hintons' Collections from South-Central Nuevo León and Adjacent Coahuila

List 3. Vascular plants from Nuevo León and adjacent Coahuila collected by James Hinton and George S. Hinton.

$\begin{array}{cl}\text { Abbreviation } & \text { Municipality } \\ \text { AN } & \text { Allende, N. L. } \\ \text { AM } & \text { Aramberri, N. L. } \\ \text { AT } & \text { Arteaga, Coah. } \\ \text { DA } & \text { Dr. Arroyo, N. L. } \\ \text { GL } & \text { Galeana, N. L. } \\ \text { IT } & \text { Iturbide, N. L. } \\ \text { LN } & \text { Linares, N. L. } \\ \text { MM } & \text { Montemorelos, N. L. } \\ \text { RA } & \text { Ramos Arizpe, Coah. } \\ \text { RY } & \text { Rayones, N. L. } \\ \text { SH } & \text { Sabinas Hidalgo, N. L. } \\ \text { SV } & \text { Salinas Victoria, N. L. } \\ \text { SC } & \text { Santa Catarina, N. L. } \\ \text { ST } & \text { Santiago, N. L. } \\ \text { VA } & \text { Villa Aldama, N. L. } \\ \text { VG } & \text { Villa de García, N. L. } \\ \text { ZR } & \text { Zaragoza, N. L. }\end{array}$

\section{ACANTHACEAE}

Anisacanthus wrightii (Torr.) A. Gray

Blechum brownei Juss.

Carlowrightia glandulosa Rob. \& Greenm.

Dyschoriste cinerascens Henrickson \& Hilsenbeck

Dyschoriste decumbens (A. Gray) O. Ktze.

Dyschoriste poliodes Leonard \& Gentry

Dyschoriste schiedeana (Nees) O. Ktze.

Elytraria bromoides Oerst.

Holographis ehrenbergiana Nees

Jacobinia incana (Nees) Hemsl.

Justicia fulvicoma Schlecht. \& Cham.

Justicia hintoniorum Nesom

Justicia pilosella (Nees) Hilsenbeck

Justicia turneri Hilsenb.

Ruellia occidentalis (A. Gray) Tharp \& Barkley

Ruellia yucatana (Leonard) Tharp \& Barkley

Siphonoglossa canbyi (Greenm.) Hilsenbeck

Stenandrium dulce (Cav.) Nees

Tetramerium nervosum Nees

Yeatesia platystegia (Torr.) Hilsenbeck

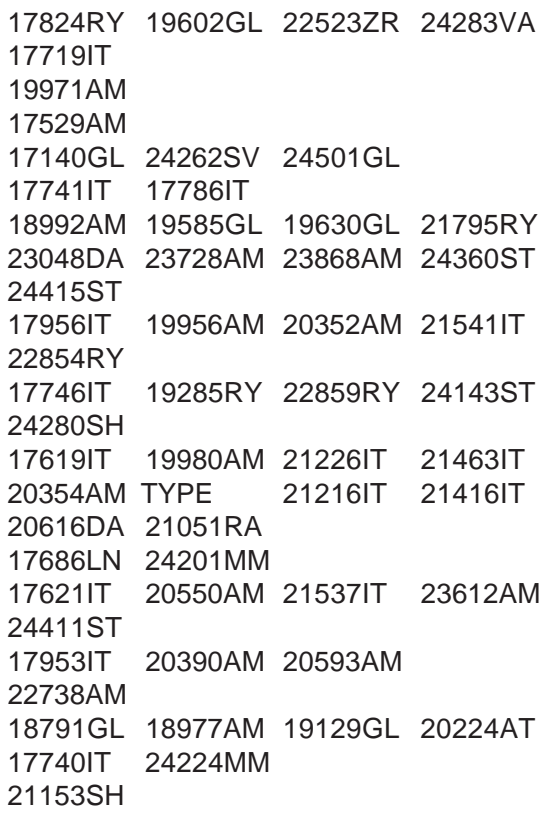

17824RY 19602GL 22523ZR 24283VA

17719IT

19971AM

17529AM

17140GL 24262SV 24501GL

17741IT 17786IT

18992AM 19585GL 19630GL 21795RY

23048DA 23728AM 23868AM 24360ST

24415ST

17956IT 19956AM 20352AM 21541IT

22854RY

17746IT 19285RY 22859RY 24143ST

24280SH

17619IT 19980AM 21226IT 21463IT

20354AM TYPE 21216IT 21416IT

20616DA 21051RA

$17686 \mathrm{LN} \quad 24201 \mathrm{MM}$

17621IT 20550AM 21537IT 23612AM

24411ST

17953IT 20390AM 20593AM

22738AM

18791GL 18977AM 19129GL 20224AT

17740IT 24224MM

21153SH 


\section{ACERACEAE}

Acer negundo L.

$21851 \mathrm{GL}$

\section{AGAVACEAE}

Agave americana ssp. protoamericana

Gentry X A. scabra

Agave celsii Hook. var. celsii

20629DA 20632DA

Agave funkiana Kunth \& Bouché

22280ZR

$18722 \mathrm{GL}$

Agave lophantha Schiede

Agave macroculmis Todaro

17809ZR

18265AT 18429AT 18444GL 18945AM

Agave scabra Salm-Dyck

Agave striata Zucc. ssp. striata

$19401 \mathrm{GL}$

19891GL 19892GL 20244GL 21099RA

Agave sp.

18986AM 19635GL

Hemiphylacus hintoniorum L. Hernández (ined.)

$19355 \mathrm{GL}$

19431RY 21186IT TYPE 22603IT

Hesperaloë funifera Trel.

Manfreda variegata Jacobi

24263SV

Manfreda sp. nov. L. Hernández

24407ST

22924AM TYPE

\section{AMARANTHACEAE}

Alternanthera caracasana Kunth

21366GL

Amaranthus hybridus L.

23502ZR

Amaranthus palmeri S. Wats.

Celosia nitida Vahl

$19507 \mathrm{GL}$

20547AM

Froelichia arizonica Thornb.

23711AM

Gomphrena decumbens Jacq.

19226GL 21187IT 21461IT

Iresine cassiniaeformis Sch.

$17748 I T$

Iresine difussa H. \& B. ex Willd.

21390IT 21660IT

Iresine heterophylla Standl.

19225GL 20022AM 20587AM

Iresine leptoclada (Hook. f.) Henrickson \& Sundb.

20830RY

Iresine orientalis Nesom

Tidestroemia lanuginosa (Nutt.) Standl.

18124GL 19286RY 22757ZR

20615DA

\section{AMARYLLIDACEAE}

Cooperia pedunculata Herb.

Hypoxis decumbens var. dolichocarpa Nesom

Hypoxis mexicana Schult.

Jaimehintonia gypsophila B. L. Turner

Zephyranthes morrisclintii Traub \& Howard

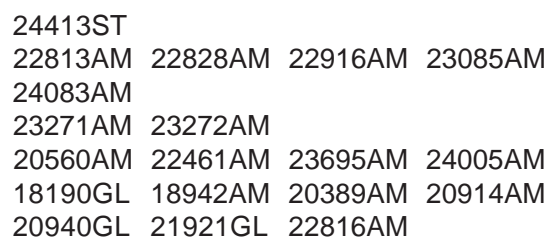


Hinton \& Hinton: Hintons' Collections from South-Central Nuevo León and Adjacent Coahuila

\section{ANACARDIACEAE}

Pistacia texana Swingle

Rhus aromatica Ait.

Rhus lanceolata (A. Gray) Britt.

Rhus microphylla Engelm. ex A. Gray

Rhus muelleri Standl. \& Barkl.

Rhus pachyrrhachis Hemsl.

Rhus trilobata Nutt.

Rhus virens Lindh. ex A. Gray

Schinus molle L.

Toxicodendron radicans (L.) Kuntze
22163GL

18801GL 19332ZR 21814IT 22643ZR

23900AM 24072AM

21234IT

18392GL 18660GL 18708GL 19381GL

21896GL 21968AM 24467GL

18082GL 19208GL 21068RA 24404ST

17473GL 21747GL 22158GL 22214ZR 23602AM

18713GL

17448DA 19228GL 21753GL 23838AM

23889AM

$24831 \mathrm{GL}$

21579GL 24124ST 24127ST

APIACEAE

Apium graveolens L.

Apium leptophyllum (Pers.) F. Muell.

Arracacia aegopodioides (Kunth) Coult. \& Rose

Arracacia hintonii Const. \& Affolter

Arracacia schneideri Math. \& Const.

Arracacia ternata Math. \& Const.

Arracacia tolucensis (Kunth) Hemsl.

Berula erecta (Huds.) Cov.

Daucus montanus Humb. \& Bonpl. ex Spreng.

Donnellsmithia ternata (S. Wats.) Math. \& Const.

Eryngium gramineum Delar. f.

Eryngium heterophyllum Engelm.

Eryngium serratum Cav.

Eryngium venustum Bartlett ex Const.

Mathiasella bupleuroides Const. \& Hitchc.

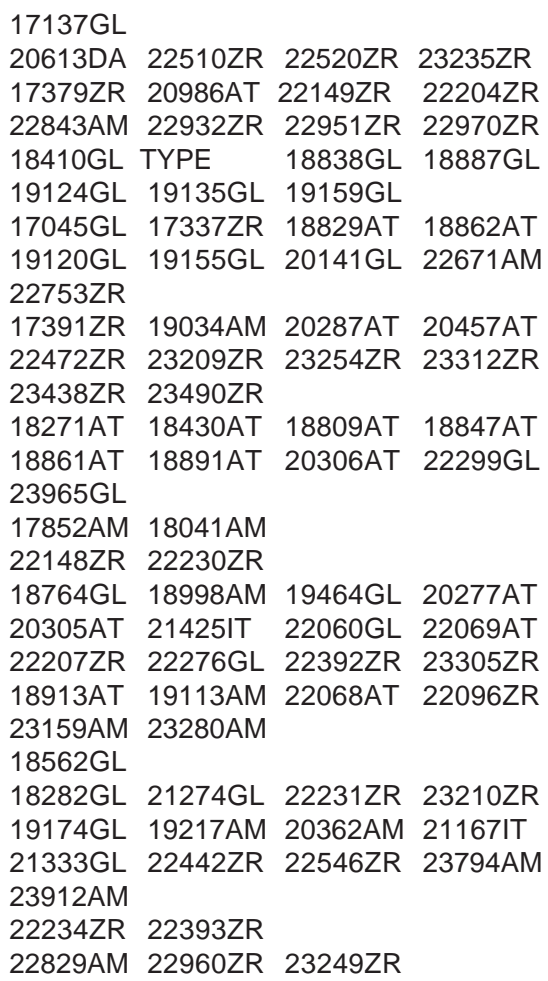


Acta Botánica Mexicana (1995), 30:41-112

Osmorhiza mexicana ssp. bipartita

(Constance \& Shan.) Lowry

Osmorhiza mexicana Griseb. ssp. mexicana

Pimpinella anisum L.

Prionosciadium humile Rose

Sanicula liberta Cham. \& Schlecht.

Tauschia hintoniorum Const. \& Affolter

Tauschia infernicola Const. \& Affolter

Tauschia johnstoniana Math. \& Const.

Tauschia madrensis Coult. \& Rose
22116ZR

21345GL 21608GL 22388ZR 22578ZR

23154AM

19482GL

$22181 \mathrm{GL} 22602 \mathrm{IT}$

21245IT 21625GL 22139ZR 22308GL

22894AM 22930AM 23155AM 23238ZR

18200AT 18426AT TYPE 18806AT

18842AT 18859AT 18886AT 18903AT

18924AT 20298AT

21810GL 23087AM

18940AM 18957AM 20151GL 20234AT

20290AT

17018GL 17330ZR 18206AT 18803AT

20971AT

\section{APOCYNACEAE}

Apocynum cannabinum L.

18485GL 24340ST

Apocynum medium var. floribundum (Greene) Woods. 17900AM

Macrosiphonia hypoleuca (Benth.) Muell. Arg.

22990ZR

Macrosiphonia lanuginosa (Mart. \& Gal.) Hemsl.

var. lanuginosa

Mandevilla karwinskii (Muell. Arg.) Hemsl.

Vinca major L.

20372AM 23009AM 24289SV

17559ZR 17948IT 19619GL 20255AT

22946ZR

19404GL

AQUIFOLIACEAE

Ilex rubra S. Wats.

22544ZR

ARACEAE

Arisaema dracontium (L.) Schott

18272ZR 22531ZR 22652AM 22739AM

\section{ARISTOLOCHIACEAE}

Aristolochia secunda Pfeifer

21939AM 22853GL

\section{ASCLEPIADACEAE}

Asclepias asperula (Dcne.) Woods.

20300AT

Asclepias bifida Blackwell

18172GL

Asclepias curassavica L.

17829RY 19936AM

Asclepias emoryi (Greene) Vail

18423GL 
Hinton \& Hinton: Hintons' Collections from South-Central Nuevo León and Adjacent Coahuila

Asclepias linaria Cav.

Asclepias mexicana Cav.

Asclepias otarioides Fourn.

Asclepias scaposa Vail

Asclepias similis Hemsl.

Asclepias subverticillata (A. Gray) Vail

Asclepias tuberosa L.

Asclepias sp.

Cynanchum barbigerum (Sch.) Shinners var. barbigerum

Cynanchum kunthii (Dcne.) Standl.

Cynanchum mccartii var. latifolium B. L. Turner ex Henrickson

Cynanchum mccartii Shinners var. mccartii

Cynanchum pringlei (A. Gray) Henrickson

Cynanchum sp.

Gonolobus sp.

Marsdenia coulteri Hemsl.

Marsdenia pringlei Wats.

Matelea lanata (Zucc.) Woods.

Matelea pilosa (Benth.) Woods.

Sarcostemma torreyi (A. Gray) Woods.

\author{
19339AM 19604GL 19921GL \\ 18423GL \\ 18758GL 20460AT \\ $19704 \mathrm{GL}$ \\ 17805ZR \\ 17135GL 17858GL 20605DA \\ 19558GL 24318ZR \\ 17428ZR \\ 17660IT 19732GL 20622DA \\ 18659GL 24347ST \\ 17935IT TYPE \\ 17776IT \\ 19616GL 19712GL 20414AT \\ 24258SV \\ 17565ZR \\ 17939IT \\ 18119IT \\ 23755AM \\ 17618IT 24181MM \\ 19008AM 24473GL
}

\section{ASTERACEAE}

Achillea millefolium $\mathrm{L}$.

Acmella oppositifolia (Lem.) Jansen var. oppositifolia

Acourtia aspera (Bacig.) Rev. \& King

Acourtia coulteri (A. Gray) Rev. \& King

Acourtia hidalgoana B. L. Turner

Acourtia hintoniorum B. L. Turner

Acourtia nana (A. Gray) Rev. \& King

Acourtia parryi (A. Gray) Rev. \& King

Acourtia purpusii (Brandeg.) Rev. \& King

Acourtia queretarana B. L. Turner

Acourtia runcinata (Lag. ex D. Don) B. L. Turner

Acourtia wrightii (A. Gray) Rev. \& King

Ageratina calaminthifolia (Kunth) King \& Robinson

Ageratina calophylla (Greene) King \& Robinson

Ageratina campylocladia (B. L. Rob.) King \& Robinson

Ageratina conspicua (King \& Robinson) King \&

Robinson

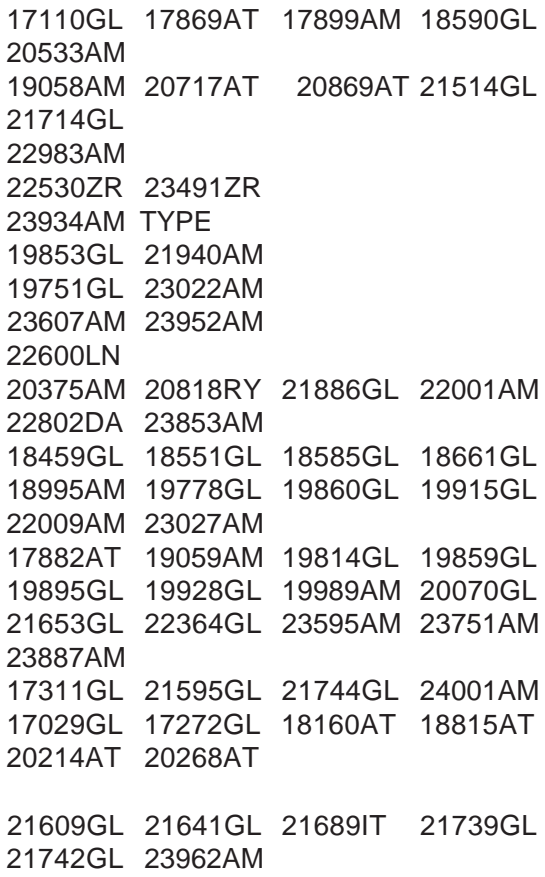

17110GL 17869AT 17899AM 18590GL 20533AM

19058AM 20717AT 20869AT 21514GL

21714GL

22983AM

22530ZR 23491ZR

23934AM TYPE

19853GL 21940AM

19751GL 23022AM

23607AM 23952AM

22600LN

20375AM 20818RY 21886GL 22001AM

22802DA 23853AM

$18459 \mathrm{GL} 18551 \mathrm{GL} 18585 \mathrm{GL} 18661 \mathrm{GL}$ 18995AM 19778GL 19860GL 19915GL 22009AM 23027AM

17882AT 19059AM 19814GL 19859GL $19895 \mathrm{GL}$ 19928GL 19989AM 20070GL

21653GL 22364GL 23595AM 23751AM 23887AM

17311GL 21595GL 21744GL 24001AM

17029GL 17272GL 18160AT 18815AT

20214AT 20268AT

21609GL 21641GL 21689IT 21739GL 21742GL 23962AM 
Ageratina espinosarum (DC.) King \& Robinson var. espinosarum

Ageratina espinosarum var. subintegrifolium

(B. L. Rob.) B. L. Turner

Ageratina gypsophila B. L. Turner

Ageratina havanensis (Kunth) King \& Robinson

Ageratina hernandezii B. L. Turner

Ageratina ilicifolia B. L. Turner

Ageratina ligustrina (DC.) King \& Robinson

Ageratina oreithales (Greenm.) B. L. Turner

Ageratina pazcuarensis (Kunth) King \& Robinson

Ageratina petiolaris (DC.) King \& Robinson

Ageratina pichinchensis (Kunth) King \& Robinson

Ageratina potosina B. L. Turner

Ageratina rollinsii B. L. Turner

Ageratina saltillensis (B. L. Rob.) King \& Robinson

Ageratina schaffneri Sch.-Bip.

Ageratina scorodonioides (A. Gray) King \& Robinson

Ageratina viburnoides (DC.) King \& Robinson

Ageratina viejoana B. L. Turner

Ageratina wrightii (A. Gray) King \& Robinson

Ageratina zapalinama B. L. Turner

Ageratina sp. nov. B. L. Turner (\#1)

Ageratina sp. nov. B. L. Turner (\#2)

Ageratum corymbosum (Zucc.) Pers.

Alloispermum scabrum (Lag.) H. Rob. var. scabrum Ambrosia confertiflora DC.

Aphanostephus ramosissimus var. humilis (Benth.)

B. L. Turner \& Birdsong

Aphanostephus ramosissimus DC. var. ramosissimus

Archibaccharis serratifolia (Kunth) Blake

Artemisia klotzschiana Besser

23783AM

17700IT 19925GL 19986AM 20789RY

21511GL 21569GL 21718GL 21763AM

18779GL TYPE 18782GL 20496AT

17443ZR 17701IT 17705IT 19096AM

19929GL 19990AM 20000AM 20840RY

1706GL 21719GL 21722GL 21730GL

21748GL 21757AM 21765AM 22004AM

22182GL 23805ZR 23886AM

23828AM

20837RY TYPE

17439ZR 22131ZR 22494ZR 22502ZR

23808ZR 23922AM

18910AT 18932AT 19075AT 22435ZR

21606GL 21610GL

19258GL 19318ZR 19354GL 19906GL

20127GL 21792RY 21818IT 24013AM

22482ZR 22515ZR 22655AM 23790AM

23954AM 24116ST

22408ZR

21761GL 23663AM

18346GL 18612GL 18709GL 19001AM

19269GL 19825GL 19903GL 20739AT

21257GL 21627GL 21652GL 21688IT

21713GL 21740GL 22446ZR 22511ZR

22517ZR

$17187 \mathrm{GL}$

17185GL 17733IT 18351GL 18928AT

19818GL 19826GL 20493AT 21590GL

17729IT 20043AM 21668IT 23829AM

23913AM

22484ZR TYPE 22504ZR 23807ZR

17465DA 18597GL 19864GL 20708AT

21738GL

20499AT TYPE

23980AM TYPE

23811ZR TYPE 23960AM 23977AM

17629ZR 19000AM 19252GL 19727GL

19850GL 19988AM 20481AT 20705AT

20794RY 22327GL 22473ZR 23710AM

23767AM

21642GL 22490ZR 23452ZR 23791AM

19595GL 20635DA

$18197 \mathrm{GL}$

17145GL 17318GL 17827RY 19497GL

19578GL 19637GL

22254ZR 23444ZR

19838GL 20763GL 
Hinton \& Hinton: Hintons' Collections from South-Central Nuevo León and Adjacent Coahuila

Artemisia ludoviciana Nutt.

Artemisia pringlei Greenm.

Aster carnerosanus S. Wats.

Aster falcatus Lindl.

Aster prealtus Poir.

Aster subulatus Michx.

Astranthium beamanii DeJong

Astranthium integrifolium var. triflorum (Rafin.) Shinners

Astranthium splendens DeJong

Aztecaster matudae (Rzed.) Nesom

Baccharis bigelovii A. Gray

Baccharis heterophylla Kunth

Baccharis neglecta Britton

Baccharis potosina A. Gray

Baccharis pteronioides DC.

Baccharis salicifolia (R. \& P.) Pers.

Baccharis salicina Torr. \& A. Gray

Baccharis sulcata DC.

Baccharis texana (T. \& G.) A. Gray

Bahia absinthifolia Benth. var. absinthifolia

Bahia absinthifolia var. dealbata (A. Gray) A. Gray

Bahia autumnalis Ellison

Bahia schaffneri S. Wats.

Berlandiera lyrata Benth. var. Iyrata

Berlandiera lyrata var. macrophylla A. Gray

Bidens angustissima Kunth

Bidens aurea (Ait.) Sherff

Bidens bigelovii A. Gray

Bidens ferulifolia (Jacq.) DC.

Bidens longistyla Hart

Bidens muelleri Sherff

Bidens odorata Cav. var. odorata

Bidens odorata var. rosea (Sch.-Bip.) Melchert

Bidens pilosa $\mathrm{L}$.

Bidens saltillensis Melchert

Bidens serrulata (Poir.) Desf.
18347GL 18576GL 19072AT 19817GL 19880GL 19918GL 20080GL 20815RY $21355 \mathrm{GL}$

$18575 \mathrm{GL}$

17247GL 17872AT 18324GL 18354AT

18619GL 19052AM 19829GL 21250GL

21431IT 21698IT 23909AM 23915AM

24009AM

$18608 \mathrm{GL}$

19932AM

17689LN 19513GL 19634GL 21481IT

$21636 \mathrm{GL}$

17006GL 18547GL 19463GL

24195LN 24416ST

17355ZR 19843GL 21248IT 21272GL

21291GL 21619GL 22192ZR 22220ZR

22953ZR 23938AM

20225AT 20231AT

19802GL 19822GL 19823GL 21517GL

21567GL 21716GL

18035AM 18066AM 18771GL

17638ZR 21538IT 23398ZR 23834AM

20440AT 20489AT 22272GL

18419GL 18656GL 18962AM 19419GL

20248GL

17968IT

20514AM 20515AM

18933AT

19040AM

18446GL 18605GL 19627GL 19708GL

19744GL 19785GL 20617DA 21506GL $21891 \mathrm{GL}$

19943AM 23014AM 23732AM

17688LN 20779RY 23678AM

21108GL 22328GL

18394GL 18672GL 19198GL 21172IT

21338GL 21368GL 23053AM

18261IT 18468GL

19018AM

19884GL

18587GL 18589GL

17634ZR

19179GL 19666GL 19764GL 20711AT

21571GL 21572GL 21708GL 23667AM $17205 \mathrm{GL}$

17446DA 17633ZR 19717GL 19718GL

19870GL 21591GL 23837AM

19641GL 19788GL 22514ZR

19787GL 21400IT 21522GL 22500ZR

20483AT

19791GL 20086GL 21614GL 
Acta Botánica Mexicana (1995), 30:41-112

Bidens triplinervia Kunth

Bidens sp.

Brickellia aramberrana B. L. Turner

Brickellia chlorolepis (W. \& S.) Shinners

Brickellia coahuilensis B. L. Turner

Brickellia conduplicata (B. L. Rob.) B. L. Rob.

Brickellia eupatorioides var. chlorolepis (W. \& S.)

B. L. Turner

Brickellia grandiflora A. Gray

Brickellia hintoniorum B. L. Turner

Brickellia laciniata A. Gray

Brickellia lemmonii var. conduplicata (B. L. Rob.) B. L. Turner

Brickellia lemmonii var. nelsonii (B. L. Rob.)

B. L. Turner

Brickellia nesomii B. L. Turner

Brickellia oliganthes (Less.) A. Gray

Brickellia palmeri A. Gray

Brickellia paniculata (Mill.) B. L. Rob.

Brickellia secundiflora var. parryi (A. Gray) B. L. Turner

Brickellia secundiflora (Lag.) A. Gray var. secundiflora

Brickellia spinulosa A. Gray

Brickellia subuligera (Schauer) B. L.Turner

Brickellia veronicaefolia Kunth

Calea ternifolia var. calyculata (B. L. Rob.) Wussow

Calyptocarpus vialis Less.

Carduus tenuiflorus Curt.

Carminatia tenuiflora DC.

Centaurea americana Nutt.

Centaurea calcitrapa L.

Chaetopappa bellioides (A. Gray) Shinners

Chaetopappa ericoides (Torr.) Nesom
18911AT 19074AT 19805GL 19844GL

21361GL 21696IT 21704IT 22385ZR

22402ZR 22441ZR 22493ZR 22545ZR

22588ZR 23453ZR

24170AN

20031AM 20050AM TYPE

$18571 \mathrm{GL}$

19030AM

$18319 \mathrm{GL}$

19812GL 19837GL 19867GL 23676AM 23684AM 23929AM

19039AM 19268GL 21280GL 21683IT

21743GL 22550ZR 22885AM 23907AM

17924AT 17970AT TYPE 18904AT

20430AT 22250ZR 22418ZR

17713IT 18042AM 19105AM 19846GL

19926GL 19950AM 19970AM 21576GL

23892AM

19924GL 20707AT 20849RY 21705GL

21727GL 21728GL 23594AM 23604AM

23661AM 23687AM 23734AM 23885AM

18610GL 18628GL 18760GL 19057AM

20348AM 20543AM 23292AM

$17257 \mathrm{GL} 18577 \mathrm{GL} 19841 \mathrm{GL}$

20366AM

18630GL 18647GL

20033AM 20039AM

23820AM

22722GL

19979AM 21682IT

17471GL 18075AM 19827GL 19848GL

19854GL 19876GL 19897GL 19968AM

20049AM 20073GL 21736GL 22572ZR

23675AM 23891AM

20586AM 21680IT

17525AM 17693LN 19794GL 19946AM

20161RY 20522AM 24082AM 24354ST

24105ST

22524ZR

17532AM 19955AM 20018AM 24438ST

$20465 \mathrm{GL}$

18990AM 21057RA 22022GL 23330GL

24460GL

18080GL 18198GL 18421GL 19130GL 19699GL 19723GL 19834GL 19857GL 19900GL 21080RA 23862AM 
Hinton \& Hinton: Hintons' Collections from South-Central Nuevo León and Adjacent Coahuila

Chaetopappa parryi A. Gray

Chaptalia hololeuca E. Greene

Chaptalia lyratifolia Burkart

Chaptalia madrensis Nesom

Chaptalia texana E. Greene

Chaptalia transiliens Nesom

Chrysactinia acerosa Blake

Chrysactinia mexicana A. Gray

Chrysactinia pinnata S. Wats.

Chrysactinia truncata S. Wats.

Cirsium acrolepis (Petrak) Ownbey

Cirsium horridulum Michx.

Cirsium pringlei A. Gray

Cirsium texanum Buckley

Cirsium undulatum $\mathrm{x}$ (Nutt.) Spreng.

Cirsium sp. nov. Nesom

Conyza canadensis (L.) Cronq.

Conyza coronopifolia Kunth

Cosmos crithmifolius Kunth

Cosmos diversifolius Otto

Cosmos sulphureus Cav.

Dahlia coccinea Cav.

Dahlia foeniculifolia Sherff

Dahlia merckii Lehm.

Dahlia tubulata Soren.

Dicranocarpus parviflorus A. Gray

Dugaldia pinetorum (Standl.) Bierner Dyssodia acerosa DC.

Dyssodia decipiens (Benth.) Johnst.

Dyssodia micropoides DC.

Dyssodia papposa (Vent.) Hitchc.
18482GL 18956AM 19017AM 19146GL

20219GL 20265AT

20114GL 20228AT 21788RY 23842AM

18955AM 20195RY 20227AT 21074RA

22737DA 23953AM

18250GL 18795GL 18958AM 20140GL

21039GL 22658AM 22750ZR 22819AM

22827AM 23091AM

20032AM 20121GL 20166RY 20805RY

21203IT 21369GL 21659IT 22594LN

23620AM 23873AM 23908AM

19685GL 20218GL 21328GL 21631GL

22003AM 22178GL

17756GL 18677GL 19610GL 22025GL

19232GL 19973AM

17569ZR 23061AM 24369ST

20403AM 21927GL 22942AM 23135AM

24229MM

21942AM

17560ZR 18909AT 19171GL 20448AT

21011AT 21031GL 21336GL 22282GL

22823AM 24339ST

19479GL 19486GL 20017AM 20365AM

20585AM 20614DA 20918AM 24236MM

17927AT 18561GL 20246GL 20470AT

20968AT

17580ZR 18917AT 20503AT 20733AT

22113ZR 23832AM

18761GL 18775GL 19515GL 19568GL

20517AM 21395IT

23211ZR 23459ZR

17413ZR 22111ZR 23289AM

22109ZR 22150ZR 22224ZR 22557ZR

23465ZR

20023AM 20065AM

19190GL 19761GL 20415AT 23095AM

23227ZR

23414ZR

17414ZR 17567ZR

17188GL 18344AT 19025AM 19178GL

21310GL 22436ZR 23152AM

18638GL 19538GL 19562GL 19638GL

19655GL 20642DA 23640ZR

$17294 \mathrm{GL}$

19280RY 19855GL 20606DA 21771RY

24353ST

24265SV

17121GL 17447DA 19539GL

19719GL 19720GL 19790GL 
Acta Botánica Mexicana (1995), 30:41-112

Dyssodia pentachaeta (DC.) B. L. Rob. var. pentachaeta

Dyssodia pinnata var. glabrescens Strother

Dyssodia pinnata (Cav.) B. L. Rob. var. pinnata

Dyssodia setifolia (Lag.) B. L. Rob. var. setifolia

Dyssodia tenuifolia (Cass.) Loes.

Engelmannia pinnatifida Nutt.

Erigeron basilobatus Blake

Erigeron bigelovii A. Gray

Erigeron calcicola Greenm.

Erigeron divergens Torr. \& A. Gray

Erigeron dryophyllus A. Gray

Erigeron flagellaris A. Gray

Erigeron gypsoverus Nesom

Erigeron hintoniorum Nesom

Erigeron metrius Blake

Erigeron modestus A. Gray

Erigeron onofrensis Nesom

Erigeron palmeri A. Gray

Erigeron potosinus Standl.

Erigeron pubescens Kunth

Erigeron veracruzensis Nesom

Eupatorium azureum DC.

Eupatorium betonicifolium Mill.

Eupatorium bigelovii A. Gray

Eupatorium collinum DC.

Eupatorium galeanum B. L. Turner

Eupatorium greggii A. Gray

Eupatorium hintoniorum B. L. Turner

Eupatorium lozanoanum Rob.

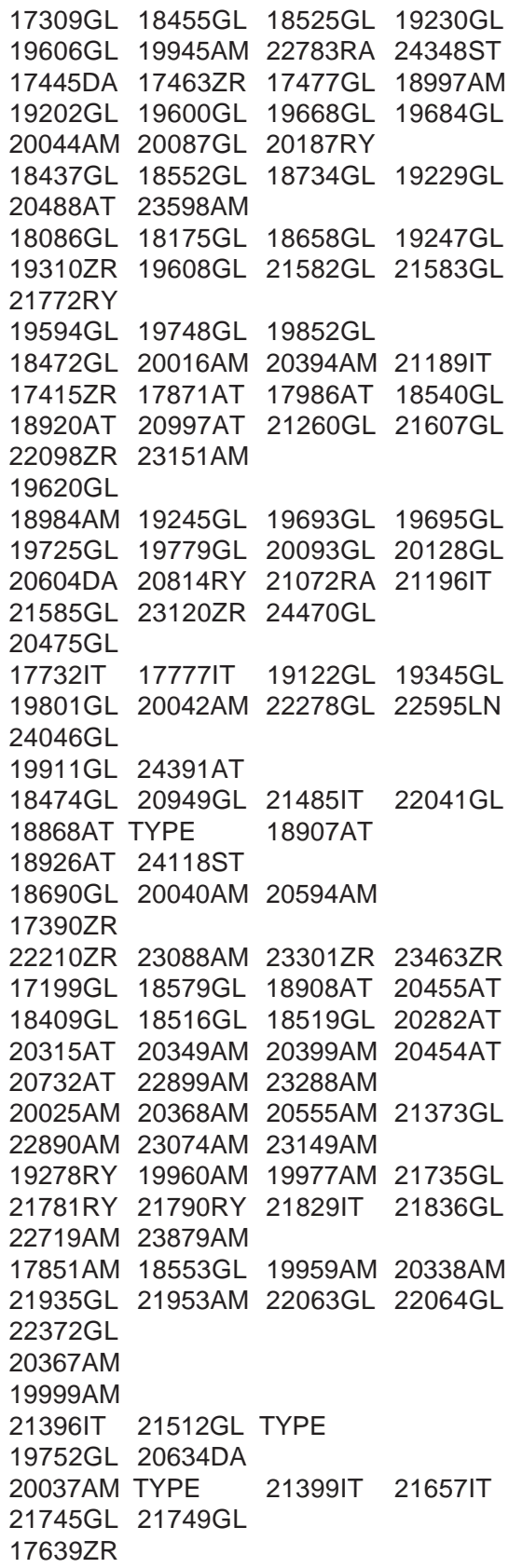

17309GL 18455GL 18525GL 19230GL 19606GL 19945AM 22783RA 24348ST

20044AM 20087GL 20187RY

$18437 \mathrm{GL} 18552 \mathrm{GL} 18734 \mathrm{GL} 19229 \mathrm{GL}$

20488AT 23598AM

18086GL 18175GL 18658GL 19247GL

19310ZR 19608GL 21582GL 21583GL

19594GL 19748GL 19852GL

18472GL 20016AM 20394AM 21189IT

18920AT 20997AT 21260GL 21607GL

22098ZR 23151AM

19620GL

18984AM 19245GL 19693GL 19695GL

20604DA 20814RY 21072RA 21196IT

21585GL 23120ZR 24470GL

20475GL

17732IT 17777IT 19122GL 19345GL

19801GL 20042AM 22278GL 22595LN

18474GL 20949GL 21485IT 22041GL

18868AT TYPE 18907AT

18926AT 24118ST

18690GL 20040AM 20594AM

17390ZR

22210ZR 23088AM 23301ZR 23463ZR

18409GL 18516GL 18519GL 20282AT

20315AT 20349AM 20399AM 20454AT

22890AM 23074AM 23149AM

19278RY 19960AM 19977AM 21735GL

21781RY 21790RY 21829IT 21836GL

17851AM 18553GL 19959AM 20338AM

21935GL 21953AM 22063GL 22064GL

$22372 \mathrm{GL}$

21396IT 21512GL TYPE

19752GL 20634DA

20037AM TYPE
21745GL 21749GL

17639ZR 
Hinton \& Hinton: Hintons' Collections from South-Central Nuevo León and Adjacent Coahuila

Eupatorium malvaefolium DC.

Eupatorium odoratum L.

Eupatorium porphyranthemum (A. Gray) King \& Robinson

Eupatorium pycnocephalum Less.

Eupatorium reyrobinsonii (B. L. Turner) B. L. Turner Eupatorium rhodochlamydeum A. Gray Eupatorium tamaulipense (King \& Robinson) B. L. Turner

Flaveria anomala B. L. Rob.

Flaveria chloraefolia A. Gray

Flaveria oppositifolia (DC.) Rydb.

Flaveria palmeri J. R. Johnston

Flaveria trinervia (Spreng.) Mohr.

Florestina pedata Cav.

Florestina tripteris DC.

Flourensia cernua DC.

Flourensia monticola Dillon

Flyriella stanfordii King \& Robinson

Gaillardia comosa A. Gray

Gaillardia mexicana A. Gray

Galinsoga parviflora Cav.

Gamochaeta falcata (Lam.) Cabr.

Gamochaeta purpurea (L.) Cabrera

Gnaphalium brachypterum DC.

Gnaphalium canescens DC.

Gnaphalium chartaceum Greenm.

Gnaphalium cryptoglandifer Nesom

Gnaphalium ehrenbergianum Sch.-Bip.

Gnaphalium flavocephalum Nesom

Gnaphalium hintoniorum Nesom

Gnaphalium liebmannii Sch.-Bip.

Gnaphalium pringlei A. Gray

Gnaphalium roseum Kunth

Gnaphalium viscosum Kunth

Gnaphalium sp.

Gochnatia hypoleuca (DC.) A. Gray

Grindelia eligulata (Steyerm.) Nesom

Grindelia greenmanii Steyerm.

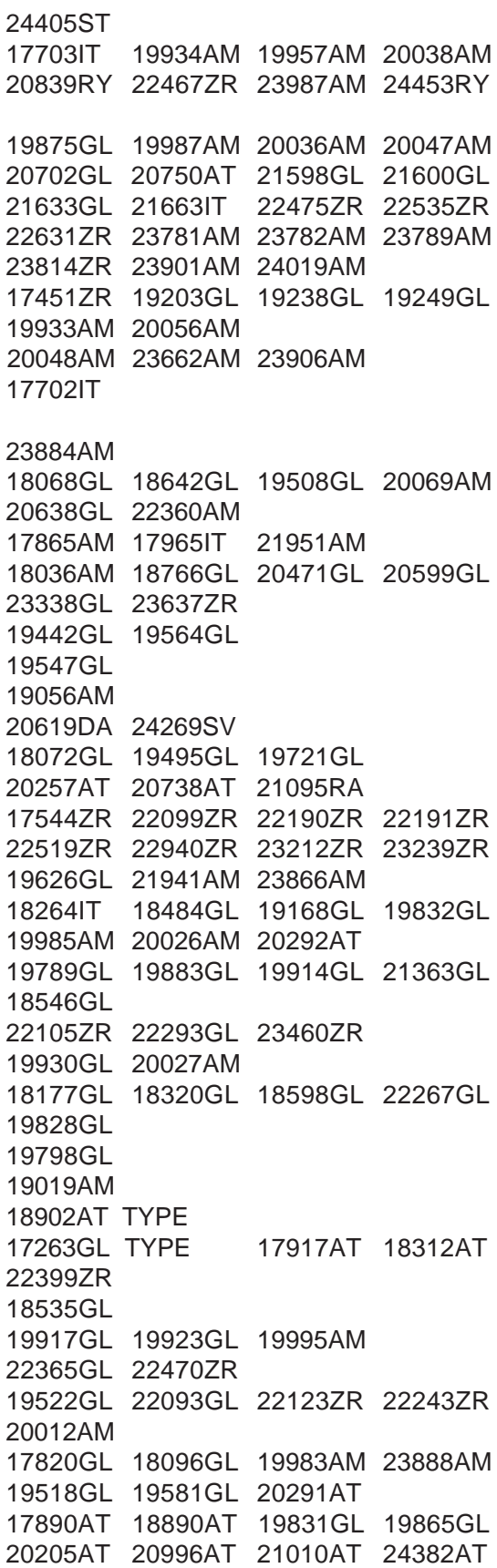


Acta Botánica Mexicana (1995), 30:41-112

Grindelia hintoniorum Nesom

Grindelia inuloides Willd. var. inuloides

Grindelia obovatifolia Blake

Grindelia turneri Nesom

Grindelia vetimontis Nesom

Gutierrezia grandis Blake

Gutierrezia microcephala (DC.) A. Gray

Gutierrezia texana var. glutinosa (Schauer) M. A. Lane

Gymnosperma glutinosum (Spreng.) Less.

Haploesthes greggii var. multiflora I. M. Johnston

Helenium elegans var. amphibolum (A. Gray) Bierner

Helenium microcephalum var. ooclinium (A. Gray) Bierner

Helianthella gypsophila var. calcarea B. L. Turner

Helianthella gypsophila B. L. Turner var. gypsophila

Helianthella quinquenervis (Hook.) A. Gray

Helianthus annuus L.

Helianthus laciniatus A. Gray

Heliopsis annua Hemsl.

Heliopsis parviflora A. Gray

Heterosperma pinnatum Cav.

Heterotheca gypsophila B. L. Turner

Heterotheca mucronata Harms ex B. L. Turner

Heterotheca subaxillaris (Lam.) Britt. \& Rusby

Hieracium abscissum L.

Hieracium dysonymum Blake

Hieracium gypsophilum B. L. Turner

Hieracium mexicanum Less.

Hieracium pringlei A. Gray

Hymenopappus flavomarginatus I. M. Johnston

Hymenopappus hintoniorum B. L. Turner

Hymenoxys linearifolia Hook.
18666GL TYPE

17148GL 17258GL 17296GL 18531GL

20735AT 20995AT 21193IT 21428IT

18618GL 20024AM 20518AM 21161IT

21231IT 21252GL 21316GL 21434IT

21446IT 22263ZR 22383ZR 22398ZR

23402ZR

17760GL 19481GL 22268GL

22434ZR

18364GL 18599GL 20232AT 20263AT

20486AT TYPE 20728AT 21096RA

17480AM 19328AM 19948AM 20637DA $22361 \mathrm{AM}$

17680LN 17932IT 18626GL 19076AM

19795GL 19871GL 19947AM 20095AM

20355AM 20607DA 21334GL

18085GL 19557GL 23824AM 23840AM 22330GL

19944AM 21232IT

24106ST 24172AN

17571ZR 20426AT 20513AT 20828RY

21367GL 24485GL

18173GL 18212GL 18246GL 18673GL

18727GL 20833GL 20956GL

18852AT 18875AT

19931AM 24169AN

17124GL 19413GL 19675GL

19530GL 19625GL 19642GL 20602DA

19485GL 20710AT

21120GL

18471GL 18476GL 18634GL 22864GL

18307AT 19139GL 19244GL 19609GL

19667GL 19833GL 19920GL 19993AM

20245GL 22074AT 22297GL 22948ZR

23286AM 24484GL

18528GL 18555GL 18646GL 19303GL

19574GL 19782GL 19797GL 19813GL

19849GL 19869GL 20096AM 20640DA

22264ZR 22366GL 24426ST

22295GL 22433ZR 23203AM

20275AT 24386AT

20239GL 22112ZR 22615ZR

18238GL 18300GL 18315AT 18317AT

$19905 \mathrm{GL}$

18439GL $18505 \mathrm{GL}$

18876AT TYPE 18918AT 18931AT

17762GL 18244GL 18404GL 18418GL

$18620 \mathrm{GL} 18629 \mathrm{GL} 18654 \mathrm{GL} 18675 \mathrm{GL}$

19842GL 20954GL 22023GL 22055GL 24335ST 
Hinton \& Hinton: Hintons' Collections from South-Central Nuevo León and Adjacent Coahuila

Hymenoxys odorata DC.

Hymenoxys scaposa (DC.) Parker var. scaposa Hymenoxys ursina Standl.

lostephane heterophylla (Cav.) Benth. Isocoma gypsophila B. L. Turner Isocoma veneta (Kunth) Greene

Jefea brevifolia (A. Gray) Strother Jefea lantanifolia (Schauer) Strother Lactuca graminifolia Michx. var. graminifolia Lactuca ludoviciana (Nutt.) Ridd.

Lactuca serriola L.

Laennecia coulteri (A. Gray) Nesom

Laennecia gnaphalioides (Kunth) Cass. Laennecia microglossa (Blake) Nesom Laennecia schiedeana (Less.) Nesom Laennecia sophiaefolia (Kunth) Nesom Leibnitzia seemannii (Sch.-Bip.) Nesom Leucactinia bracteata (Wats.) Rydb. Liatris punctata Hook. Machaeranthera crutchfieldii B. L. Turner Machaeranthera heterophylla R. Hartm. Machaeranthera pinnatifida var. chihuahuana B. L. Turner \& Hartman

Machaeranthera pinnatifida (Hook.) Shinners var. pinnatifida

Machaeranthera tanacetifolia (Kunth) Nees

Melampodium longifolium Cav.

Melampodium montanum Benth. var. montanum

Melampodium montanum var. viridulum Stuessy

Melampodium perfoliatum (Cav.) Kunth

Mikania micrantha Kunth

Montanoa arborescens (DC.) Sch.-Bip.

Montanoa tomentosa Cerv. var. tomentosa

Parthenium argentatum A. Gray

Parthenium bipinnatifidum (Ort.) Rollins

Parthenium confertum var. lyratum (A. Gray) Rollins

Parthenium incanum Kunth

Pectis angustifolia var. tenella (DC.) Keil

Pectis tenuifolia (Cass.) Less.

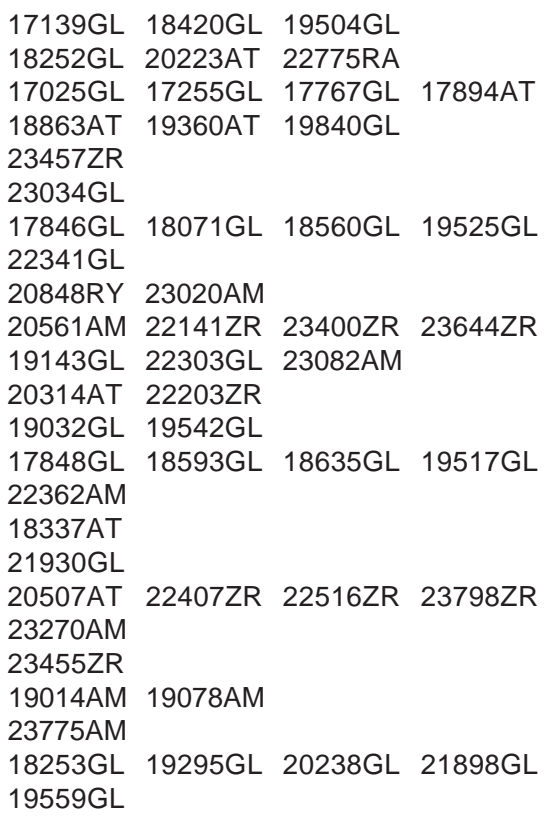


Perymenium hintoniorum B. L. Turner

Perymenium tamaulipense B. L. Turner

Pinaropappus roseus var. maculatus McVaugh

Pinaropappus roseus var. mcvaughii Poole

Pinaropappus roseus (Less.) Less. var. roseus

Piqueria trinervia Cav.

Pluchea carolinensis (Jacq.) G. Don

Pluchea odorata (L.) Cass.

Pluchea salicifolia (Mill.) Blake

Polymnia maculata Cav.

Porophyllum amplexicaule Engelm. \& A. Gray

Porophyllum coloratum (Kunth) DC. var. coloratum

Porophyllum filiforme Rydb.

Porophyllum scoparium A. Gray

Psacalium peltatum var. adenophorum Blake

Psacalium radulifolium (Kunth) Rob. \& Brett.

Psilactis asteroides A. Gray

Psilactis brevilingulata Sch.-Bip. ex Hemsl.

Psilactis tenuis S. Wats.

Psilostrophe gnaphalodes DC.

Pyrrhopappus parviflorus DC.

Pyrrhopappus pauciflorus (D. Don) DC.

Ratibida columnaris (Sims) D. Don

Rumfordia alcortae Rzed.

Rumfordia exauriculata B. L. Turner

Sanvitalia angustifolia A. Gray

Sanvitalia ocymoides DC.

Sartwellia mexicana A. Gray

Sclerocarpus uniserialis var. frutescens (Brandeg.) Feddema

Senecio aschenbornianus Schauer

Senecio bellidifolius Kunth

Senecio carnerensis Greenm.

Senecio carnerosanus S. Wats.

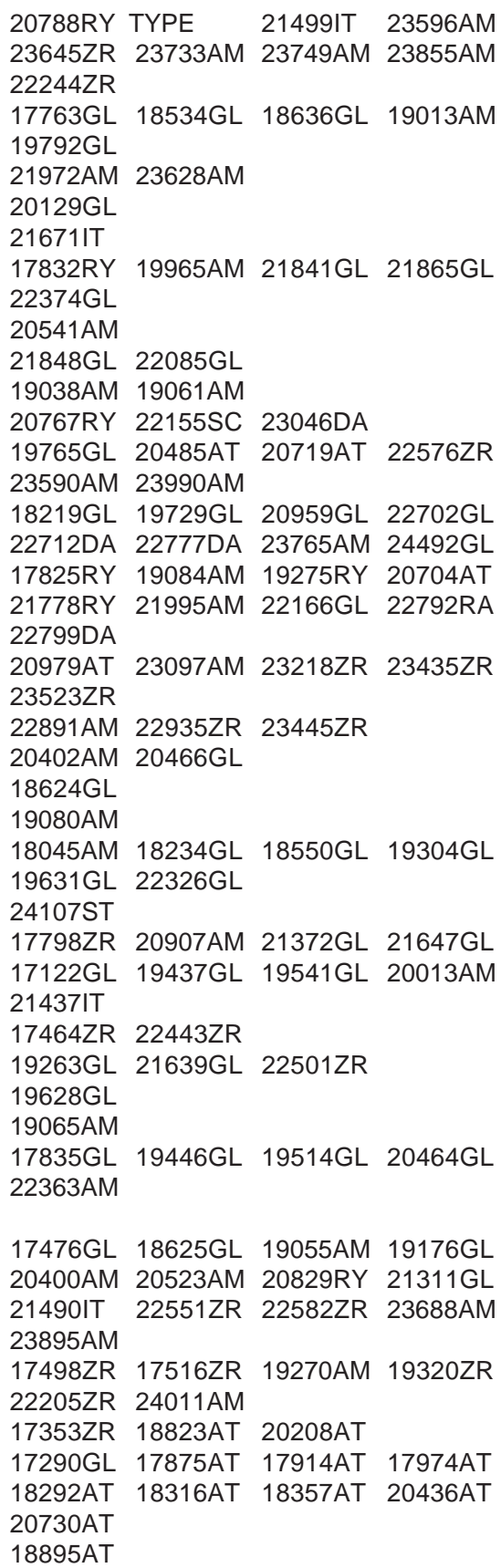


Hinton \& Hinton: Hintons' Collections from South-Central Nuevo León and Adjacent Coahuila

Senecio coahuilensis Greenm.

Senecio hintoniorum B. L. Turner

Senecio leonensis Greenm.

Senecio loratifolius Greenm.

Senecio madrensis A. Gray

Senecio monterreyanus S. Wats.

Senecio nesomiorum B. L. Turner

Senecio octobracteatus B. L. Turner \& Barkley

Senecio picridis Schauer

Senecio platanifolius Benth.

Senecio platypus Greenm.

Senecio powellii B. L. Turner

Senecio radulifolius (Kunth) Sch.-Bip.

Senecio richardsonii B. L. Turner

Senecio salignus DC.

Senecio sundbergii B. L. Turner

Senecio tampicanus DC.

Senecio toluccanus DC.

Senecio viejoanus B. L. Turner (ined.)

Senecio vulgaris $\mathrm{L}$.

Senecio vulneraria DC.

Senecio zimapanicus Hemsl.

Simsia amplexicaulis (Cav.) Pers.

Simsia calva (A. Gray) A. Gray

Smallanthus uvedalius (L.) Mackenzie

Solidago ericamerioides Nesom

Solidago gigantea Ait.

Solidago hintoniorum Nesom

Solidago orientalis (Nesom) Nesom

Solidago pringlei Fern.

Solidago simplex Kunth

Solidago velutina DC.

Sonchus oleraceus L.

Sonchus tenerrimus L.

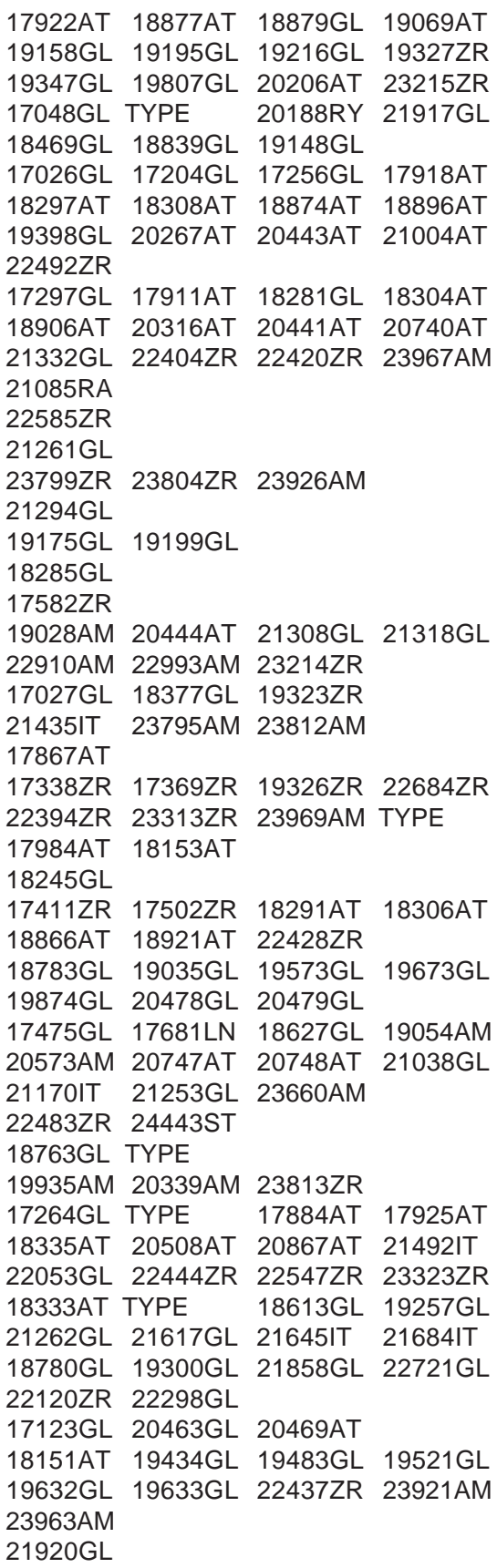


Stevia berlandieri A. Gray var. berlandieri

Stevia berlandieri var. podadenia B. Rob.

Stevia hintoniorum B. L. Turner

Stevia incognita Grashoff

Stevia jorullensis Kunth

Stevia origanoides Kunth

Stevia ovata var. expansa Grashoff

Stevia ovata Willd. var. ovata

Stevia pilosa Lag.

Stevia rhombifolia Kunth

Stevia salicifolia Cav. var. salicifolia

Stevia serrata Cav. var. serrata

Stevia tephra B. L. Rob.

Stevia tomentosa Kunth

Stevia viscida Kunth

Steviopsis nesomii B. L. Turner

Tagetes lucida Cav.

Tanacetum parthenium (L.) Sch.-Bip.

Taraxacum officinale $\mathrm{L}$.

Tetrachyron grayi (Klatt) Wussow \& Urb.

Tetrachyron tomentosum (A. Gray) Wussow

Thelesperma filifolium (Hook.) A. Gray var. filifolium

Thelesperma filifolium var. megacarpum Melchert

Thelesperma graminiforme (Sherff) B. L. Turner

Thelesperma longipes A. Gray

Thelesperma megapotamicum (Spreng.) O. Ktze. var. megapotamicum

Thelesperma scabridulum Blake

Thelesperma simplicifolium var. macrocarpum Melchert
19005AM 19051AM 19803GL 20353AM

20570AM 21178IT 21197IT 21593GL

21651GL 21667IT 21741GL 22054GL

22058GL 22902AM 23138AM 24162ST

$24475 \mathrm{GL}$

22247ZR

21611GL TYPE 21626GL 21664IT

22101ZR 22108ZR 22219ZR 22413ZR

23428ZR 23429ZR 23914AM

21702IT 21710GL 22471ZR 22485ZR

22512ZR 22584ZR 23788AM 23810ZR

17118GL 19073AT 19919GL 22521ZR

22552ZR 22583ZR

17427ZR

21628GL 21650GL 22508ZR 23681AM

23786AM 23796ZR 23944AM

19991AM 20030AM 21670IT 21691IT

21700IT 22130ZR 22474ZR 22498ZR

22518ZR 23588AM

17409ZR 18912AT 19816GL 19836GL

20511AT 20581AM 22209ZR 22236ZR

23281AM

17423ZR

18460GL 18591GL 19845GL 19909GL

20075GL 20149GL 20213AT 20497AT

21504IT 21592GL 22059GL 22424ZR

22497ZR

19248GL 21243IT 21340GL 21601GL

22606GL 23234ZR 23924AM

17426ZR 19262GL 22495ZR

18592GL 19815GL 20706AT 21637GL

23779AM 23780AM

22127ZR

22447ZR 23947AM 23958AM 24031AM

17878AT 18325GL 18578GL 18617GL

19806GL 21324GL

22246ZR

19363AT 19410GL

23106AM

17558ZR

20945GL

23130ZR

17346ZR

18518GL 18983AM 20393AM 21979AM

22269GL 22315GL 22348AM

20250GL

19533GL 22342GL

21494IT 21759AM 22039GL 22347AM

23111ZR 23170AM 23735AM 23864AM 
Hinton \& Hinton: Hintons' Collections from South-Central Nuevo León and Adjacent Coahuila

Thelesperma simplicifolium A. Gray var. simplicifolium

Tithonia tubiformis (Jacq.) Cass.

Townsendia mexicana A. Gray

Tridax coronopifolia Kunth

Tridax hintoniorum B. L. Turner

Tridax procumbens $\mathrm{L}$.

Trigonospermum annuum McVaugh \& Lask.

Trixis angustifolia DC.

Trixis californica Kellog var. californica

Trixis inula Crantz

Verbesina aramberrana B. L. Turner

Verbesina chihuahuensis A. Gray

Verbesina coahuilensis A. Gray

Verbesina daviesiae B. L. Turner

Verbesina aff. daviesiae B. L. Turner

Verbesina encelioides (Cav.) A. Gray

Verbesina hintoniorum B. L. Turner

Verbesina hypoglauca Sch.-Bip. ex Klatt

Verbesina hypomalaca var. gypsophila B. L. Turner Verbesina hypomalaca var. saltillensis B. L. Turner

Verbesina longipes Hemsl.

Verbesina microptera DC.

Verbesina mollis Kunth

Verbesina olsenii B. L. Turner

Verbesina persicifolia DC.

Verbesina virginica $\mathrm{L}$.

Verbesina zaragozana B. L. Turner

Vernonia greggii var. ervendbergii (A. Gray) B. L.

Turner

Vernonia greggii A. Gray var. greggii

Vernonia obtusa (Gleason) Blake var. obtusa

Vigethia mexicana (Wats.) Weber

Viguiera adenophylla Blake
18728GL 19012AM 20404AM 20516AM 21090RA

18644GL 18651GL 20019AM

17908AM 18834GL 19224GL 19749GL

20107GL 20620DA

24277SH

21566GL 22031GL TYPE 22061GL

21769AM 24278SH

23419ZR 23476ZR 23478ZR 23493ZR

22713DA 22800DA

19085AM 21117GL 21984AM

20021AM

21963AM 21975AM 22359AM TYPE

23638ZR

22789RA

19212GL 19236GL 20422AT 21034GL

21087RA 23669AM 24367ST

19041AM 20319AT 20421AT 20439AT

20484AT 20495AT 22006AM

18914AT

17847GL 18679GL 19100AM 19441GL

19548GL 19569GL 22184SC

$18611 \mathrm{GL} 18756 \mathrm{GL} 18778 \mathrm{GL} 19243 \mathrm{GL}$

20850GL 21581GL 23514ZR 23632ZR

23702AM

17429ZR 19264GL 21354GL 22384ZR

22503ZR 23806ZR 23951AM

20746AT

18529GL 18980AM 19615GL 19670GL

19671GL 19784GL 22079AT 23075AM

$17842 \mathrm{GL} 18229 \mathrm{GL} 18716 \mathrm{GL}$ 18732GL

$18750 \mathrm{GL} 19589 \mathrm{GL} 19771 \mathrm{GL} 21106 \mathrm{GL}$

22030GL 22057GL 23019AM 23625AM

18353IT 21168IT

19201GL 20771RY 21438IT 21655IT

$18645 \mathrm{GL}$ 19053AM 19520GL 21313GL

20846RY 21414IT 21673IT 22465ZR

23399ZR 23443ZR 23634ZR 23777AM

23880AM

17425ZR

18093GL 20825RY 21762AM

20408AM 20545AM 20553AM 22132ZR

24114ST 24117ST

17111GL 17400ZR 18481GL 18665GL 19321ZR 19835GL 21568GL 22078AT

17424ZR 17704IT 19010AM 19036AM

20045AM 23411ZR 23897AM

20028AM 20360AM24104ST

19043AM 19050AM 21342GL 21375GL

21707GL 23431ZR 
Viguiera cordata (H. \& A.) D'Arcy

Viguiera cordifolia A. Gray

Viguiera dentata (Cav.) Spreng.

Viguiera greggii (A. Gray) Blake

Viguiera multiflora (Nutt.) Blake Viguiera potosina Blake

Viguiera stenoloba Blake var. stenoloba

Wedelia ayerscottiana B. L. Turner

Wedelia hispida Kunth

Xanthium strumarium L.

Xylothamia riskindii (B. L. Turner) Nesom

Zaluzania megacephala Sch.-Bip. var. megacephala

Zaluzania mollissima A. Gray

Zaluzania parthenioides (DC.) Rzed.

Zaluzania trilobata (Ort.) Pers.

Zinnia acerosa (DC.) A. Gray var. acerosa

Zinnia juniperifolia (DC.) A. Gray

Zinnia peruviana L.

BASELLACEAE

Anredera scandens (L.) Moq.

BEGONIACEAE

Begonia gracilis Kunth

Begonia uniflora S. Wats.

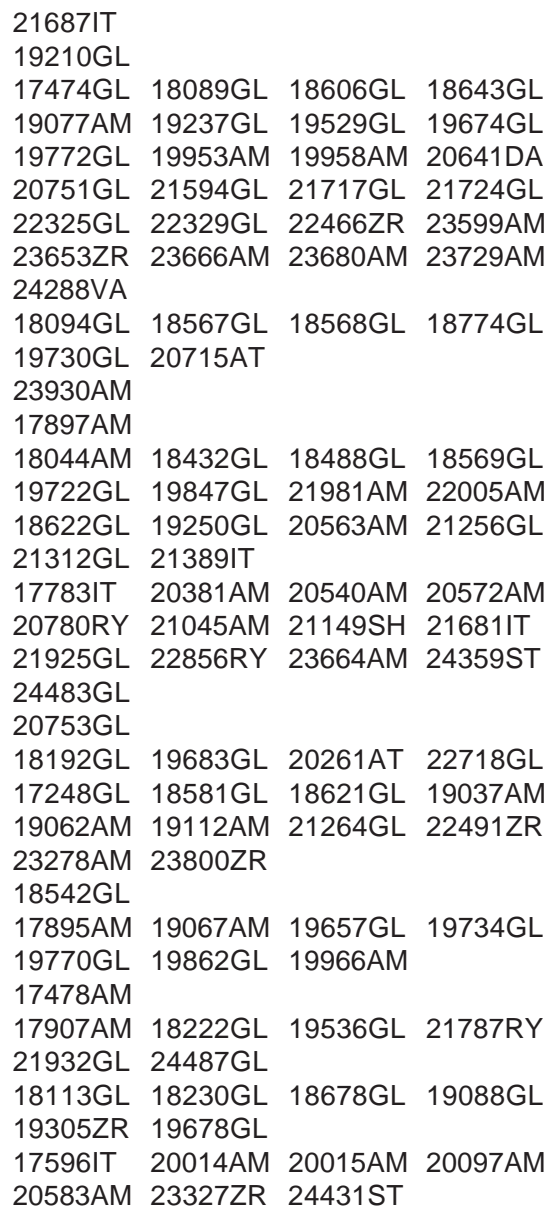


Hinton \& Hinton: Hintons' Collections from South-Central Nuevo León and Adjacent Coahuila

\section{BERBERIDACEAE}

Berberis eutriphylla (Fedde) C. G. Muller

20113GL

Berberis gracilis var. madrensis Marroq.

17494ZR 17495ZR 17721IT 19220AM

Berberis trifoliolata Moric.

20137GL

18386GL 19450GL

\section{BETULACEAE}

Ostrya virginiana (Mill.) K. Koch

22124ZR 22307GL

\section{BIGNONIACEAE}

Chilopsis linearis (Cav.) Sweet

17781IT

Tecoma stans (L.) Juss.

17823RY 19433RY 19650GL

BORAGINACEAE

Antiphytum heliotropioides DC.

Antiphytum hintoniorum Higgins \& B. L. Turner

Cordia boissieri DC.

Cordia podocephala Torrey

Cryptantha albida (Brandeg.) I. M. Johnston

Cryptantha mexicana (Brandeg.) I. M. Johnston

Cynoglossum henricksonii Higgins

Ehretia anacua (Berl.) I. M. Johnston

Hackelia leonotis I. M. Johnston

Heliotropium angiospermum Murray

Heliotropium calcicola Fern.

Heliotropium confertifolium (Torr.) A. Gray

Heliotropium curassavicum L.

Heliotropium fruticosum L.

Heliotropium procumbens Mill.

Heliotropium torreyi I. M. Johnston

Lithospermum calcicola B. L. Rob.

Lithospermum distichum Ort.

Lithospermum hintoniorum B. L. Turner (ined.)

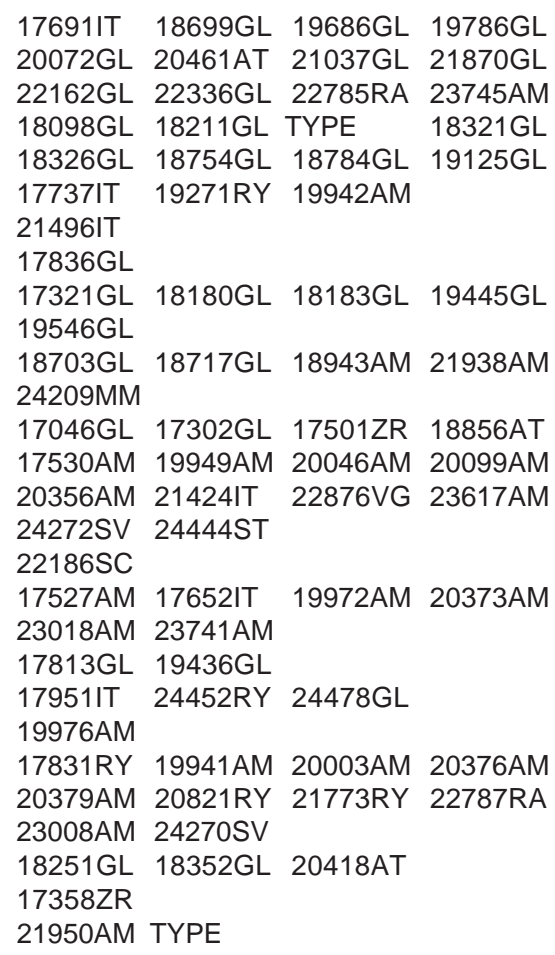

17691IT 18699GL 19686GL 19786GL 20072GL 20461AT 21037GL 21870GL 22162GL 22336GL 22785RA 23745AM 18098GL 18211GL TYPE 18321GL 18326GL 18754GL 18784GL 19125GL 17737IT 19271RY 19942AM 21496IT 17836GL 17321GL 18180GL 18183GL 19445GL 19546GL 18703GL 18717GL 18943AM 21938AM 24209MM

17046GL 17302GL 17501ZR 18856AT

17530AM 19949AM 20046AM 20099AM 20356AM 21424IT 22876VG 23617AM 24272SV 24444ST 22186SC

17527AM 17652IT 19972AM 20373AM 23018AM 23741AM

17813GL 19436GL 17951IT 24452RY 24478GL 19976AM

17831RY 19941AM 20003AM 20376AM 20379AM 20821RY 21773RY 22787RA 23008AM 24270SV $18251 \mathrm{GL}$ 18352GL 20418AT 17358ZR

21950AM TYPE 
Lithospermum indecorum I. M. Johnston Lithospermum matamorense DC.

Lithospermum muelleri I. M. Johnston

Lithospermum parksii var. rugulosum I. M. Johnston

Lithospermum viride E. Greene

Lithospermum sp.

Macromeria barbigera I. M. Johnston

Macromeria leonotis I. M. Johnston

Macromeria notata I. M. Johnston

Omphalodes aliena A. Gray

Omphalodes cardiophylla A. Gray ex Hemsl.

Omphalodes erecta I. M. Johnston

Omphalodes mexicana S. Wats.

Onosmodium dodrantale I. M. Johnston

Tiquilia canescens (DC.) A. Richardson

Tiquilia greggii (Torr. \& A. Gray) A. Richardson

Tiquilia purpusii (Brandeg.) A. Richardson

\author{
18128GL 22830AM 22926AM \\ 21821IT 21868GL \\ $18247 \mathrm{GL}$ \\ 18120IT 18143IT 18802GL 20118GL \\ 21839GL 21850GL 21908GL \\ 17753GL 18718GL 18721GL 20181RY \\ 20288AT 22072AT \\ 22729AM \\ 21351GL 22912AM \\ 17412ZR 17566ZR 18523GL 18970AM \\ 19375GL 21026GL 22277GL 23060AM \\ 18287AT \\ 20173RY 22773RA \\ 19118GL 19137GL 21027GL 22768RA \\ 24342ST 24454RY \\ 19196GL 21247IT 22275GL 23142AM \\ 21036GL \\ 17194GL 17378ZR 22117ZR 23253ZR \\ 18232GL 20765GL 21980AM 23002AM \\ 23001AM \\ 23869AM
}

\section{BRASSICACEAE}

Asta schaffneri var. pringlei (Schulz) Rollins

Asta schaffneri (S. Wats.) Schulz var. schaffneri

Brassica rapa var. campestris (L.) Koch

Cardamine auriculata S. Wats.

Cardamine macrocarpa Brandeg.

Cibotarium macropetalum Rollins

Cibotarium macrum (Standl.) B. L. Rob.

Descurainia pinnata (Walt.) Britton

Descurainia virletii (Fourn.) Schulz

Diplotaxis muralis (L.) DC.

Draba cuneifolia Torr. \& A. Gray var. cuneifolia

Draba helleriana E. Greene

Erysimum asperrimum (Greene) Rydb.

Erysimum capitatum (Dougl. ex Hook.) Greene ssp. capitatum

Lepidium lasiocarpum Nutt.

Lepidium montanum var. alyssoides (A. Gray) M. E. Jones

Lepidium virginicum $\mathrm{L}$.

Lesquerella argyraea var. diffusa (Rollins) B. L. Turner

Lesquerella berlandieri S. Wats.
18081GL 18744GL 20193RY 20490AT

21548IT 23589AM

18503GL 21796GL

18118IT 18140IT

19351GL 20209GL 21813GL

18499GL 20831RY 21852GL

18138GL 20009AM 21455IT

18817AT 20117GL

18334AT

19082AM 19505GL 22008AM

18130GL 20104GL 20116GL

17033GL 17037GL 17050GL 17331ZR

17384ZR 17973AT 19391GL 22425ZR

21782RY 22419ZR 24344ST

17034GL 17386ZR 17913AT 18443GL 18458GL 18662GL 18845AT 20147GL 20259AT 20303AT

$19490 \mathrm{GL}$

17838GL 19223GL 19424GL

20115GL

18452GL 18454GL 19607GL 19700GL 20101GL 20123GL 20162RY 21102GL 21776RY

20006AM 20342AM 
Hinton \& Hinton: Hintons' Collections from South-Central Nuevo León and Adjacent Coahuila

Lesquerella fendleri (A. Gray) S. Wats.

Lesquerella inflata Rollins \& Shaw

Lesquerella mirandiana Rollins

Nasturtium officinale $\mathrm{R}$. Br.

Nerisyrenia camporum (A. Gray) Greene

Nerisyrenia gracilis I. M. Johnston

Nerisyrenia linearifolia (Wats.) Greene

Nerisyrenia mexicana (Bacon) B. L. Turner

Pennellia longifolia (Benth.) Roll.

Raphanus sativus $\mathrm{L}$.

Rapistrum rugosum (L.) All.

Rorippa nasturtium-aquaticum (L.) Hayek

Scoliaxon mexicanus (Wats.) Payson

Sibara runcinata (Wats.) Rollins

Sisymbrium auriculatum A. Gray

Synthlipsis greggii A. Gray

Thelypodiopsis arcuata Rollins

Thelypodiopsis purpusii (Brandeg.) Rollins

Thelypodiopsis versicolor (Brandeg.) Rollins

Thelypodium longipes (Rollins) Rollins

Thelypodium wrightii A. Gray

Thlaspi mexicanum Standl.
17287GL 18497GL 18833GL 19428GL $19501 \mathrm{GL}$ 22770RA 18501GL 18973AM 21071RA 21904GL $22165 \mathrm{GL}$ $18069 \mathrm{GL}$

18226GL 19337AM 19444GL 19524GL 21947AM

18134GL 19653GL 19680GL 20171RY $21804 \mathrm{GL}$ 23843AM

17980AT 18303AT 18867AT 18892AT 18901AT 20447AT 22415ZR 23261ZR 17132GL 19500GL

20253AT

17575ZR 17769GL 22651AM

20164RY 21784RY 21838GL

19505GL 20103GL 21110GL

18174GL 18466GL 18586GL 20088GL 18142IT 18188GL 21820IT 21840GL 20722AT 21869GL 23245ZR 19707GL 20310AT 20812RY 18336AT 18899AT 19188GL 19189GL 19205GL 21003AT 21035GL 17021GL 17203GL 18824AT 19392GL 20991AT

\section{BROMELIACEAE}

Hechtia glomerata Zucc.

Tillandsia bartramii Ell.

Tillandsia benthamiana Klotz.

Tillandsia parryi Baker

Tillandsia pringlei S. Wats.

Tillandsia recurvata $\mathrm{L}$.

Tillandsia usneoides L.
20916AM

21259GL 22901AM 22988ZR

17341ZR 17773GL 18686GL 20944GL $21251 \mathrm{GL} 21359 \mathrm{GL} 21880 \mathrm{GL}$ $21831 \mathrm{GL}$

20934AM 22855RY 23847AM

19596GL

23899AM

\section{CACTACEAE}

Aztekium hintonii Glass \& Fitz.

Echinocactus horizonthalonius Lemaire

Echinocereus knippelianus Liebner

Echinocereus pentalophus (DC.) Lemaire var. pentalophus

Echinocereus rayonensis N. P. Taylor

Ferocactus pilosus (Gal. ex Salm-Dyck) Werdermann
21495IT 21528GL TYPE 21529GL $18178 \mathrm{GL}$ 18938AT

18144IT 21797RY

22637ZR

$18165 \mathrm{GL}$ 
Geohintonia mexicana Glass \& Fitz.

Mammillaria glassii R. Foster

Mammillaria magnimamma Haworth

Mammillaria rubrograndis Reppenhagen \& Lau

Mammillaria uncinata Zucc. ex Pfeiff.

Opuntia imbricata (Haw.) DC. var. imbricata

Opuntia leptocaulis DC.

Opuntia phaeacantha Engelm.

Opuntia stenopetala Engelm. var. stenopetala

Stenocactus violaciflorus (Quehl) Britt.

Turbinicarpus gautii (L. Benson) A. Zimmermann

Turbinicarpus sp. nov. Glass \& Fitz. (\#1)

Turbinicarpus sp. nov. Glass \& Fitz. (\#2)

\author{
21519GL 21527GL TYPE \\ 20973AT 21016GL \\ $18934 G L$ \\ 22646ZR \\ $18808 \mathrm{GL}$ \\ 17840GL $18835 \mathrm{GL}$ \\ 17718IT 17860GL 20626DA \\ $17839 \mathrm{GL} 17841 \mathrm{GL} \quad 18837 \mathrm{GL}$ \\ $18726 \mathrm{GL}$ \\ $18796 \mathrm{GL}$ \\ 18379GL 18818AT \\ 21805GL TYPE \\ 24010GL TYPE
}

\section{CAMPANULACEAE}

Campanula rotundifolia $\mathrm{L}$.

Lobelia berlandieri DC. var. berlandieri

Lobelia calcarea Wimmer

Lobelia cardinalis ssp. graminea (Lam.) McVaugh

Lobelia divaricata Hook. \& Arn.

Lobelia ehrenbergii Vatke var. ehrenbergii

Lobelia gypsophila Ayers

Lobelia margarita Wimmer

Lobelia subulibera S. Wats.

Lobelia trivialis Wimmer
17105GL 17306GL 17923AT 18583GL 20449AT 21282GL 22288GL 22580ZR 23522ZR

17422ZR

20170RY

19961AM 20067AM 21556IT 23291AM $17315 \mathrm{GL}$ 20734AT

19307ZR 19330AM 19691GL 20388AM 20597AM 23116ZR 23333GL 23988AM $18101 \mathrm{GL} 18746 \mathrm{GL} 21580 \mathrm{GL} 21721 \mathrm{GL}$ 21867GL 21962AM 22316GL

17534ZR 21371GL 21703IT 22300GL 22626ZR 22686ZR 22887AM 22906AM 23927AM 24129ST 24234MM

18277GL 19233GL 22161GL 23397ZR 24482GL

\section{CAPPARIDACEAE}

Polanisia uniglandulosa (Cav.) DC.

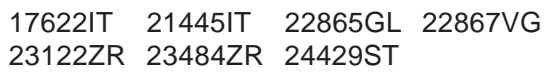

\section{CAPRIFOLIACEAE}

Abelia coriacea Hemsl.

19266GL

Lonicera albiflora T. \& G.

Lonicera pilosa (Kunth) Willd.

18358AT $18406 \mathrm{GL}$

17127GL 17370ZR 18724GL 20270AT

Sambucus caerulea Raf.

23262ZR 23320ZR

17362ZR 17539AM 18362AT 20988AT

Sambucus mexicana Presl

22815AM

I24175AN 
Hinton \& Hinton: Hintons' Collections from South-Central Nuevo León and Adjacent Coahuila

Symphoricarpos microphyllus Kunth

17115GL 17387ZR 18293AT 18343AT 20992AT

Viburnum rafinesquianum var. affine (Schn.) House 18683GL

\section{CARYOPHYLLACEAE}

Arenaria bourgaei Hemsl.

17333ZR

Arenaria gypsostrata B. L. Turner

20383AM 22007AM 23706AM TYPE 23998AM

Arenaria hintoniorum B. L. Turner 20957GL TYPE

Arenaria lanuginosa (Michx.) Rohrb. var. lanuginosa 22622ZR 22821AM 23279AM

Arenaria lanuginosa var. saxosa (A. Gray) B. L. Turner18814AT 21871GL 22252ZR 22410ZR

Arenaria lycopodioides Willd.

24015AM

17909AM 18532GL 18741GL 19713GL

20861AT 22020GL 22822AM

Arenaria oresbia Greenm.

17009GL 17291GL 17909AM 17910AT

Cerastium brachypodum (Engelm. ex A. Gray) Robinson

Drymaria anomala S. Wats.

Drymaria gracilis Cham. \& Schlecht.

18827AT 18853AT 21134GL

23387GL

23466ZR

$21135 \mathrm{GL}$

$18129 \mathrm{GL}$

Drymaria villosa Cham. \& Schlecht.

24341ST

Saponaria officinalis $\mathrm{L}$.

Silene laciniata ssp. greggii (A. Gray) Hitchc.

17196GL 17696LN 17981AT 18905AT

19070AM 19164GL 19197GL 20429AT

20741AT 21288GL 22396ZR

Stellaria cuspidata Willd.

18122IT 20217GL 20241GL 22386ZR

22669AM 22919AM 23801ZR 24112ST

Stellaria hintoniorum B. L. Turner

17040GL 17108GL TYPE 17389ZR

18869AT 20307AT 24017AM

\section{CELASTRACEAE}

Maytenus phyllanthoides Benth.

17845GL 18790AM 21956AM

Mortonia greggii A. Gray

17817GL

Mortonia palmeri Hemsl.

18399GL 18403GL 18415GL 18695GL

Orthosphenia mexicana Standl.

19754GL 21899GL 21964AM

18067AM

Pachistima myrsinites ssp. mexicana Navarro

18158AT 18811AT 19452GL

CHENOPODIACEAE

Atriplex canescens (Pursh) Nutt.

Atriplex muricata H. \& B. ex Willd.

Atriplex reptans I. M. Johnston

Chenopodium ambrosioides L.

Chenopodium berlandieri Moq.

Chenopodium fremontii Wats.
18777GL 19420GL 19487GL $20701 \mathrm{GL}$ 23039GL

17608IT

18440GL 19498GL 20806RY

19207GL 
Chenopodium graveolens L.

Chenopodium incisum Poir.

Chenopodium murale L.

Salsola australis $\mathrm{R}$. Br.
19656GL 19747GL 19913GL

23499ZR 23673AM

20220GL

19509GL 19677GL

\section{CISTACEAE}

Helianthemum glomeratum (Lag.) Lag. ex Dunal 21634GL

\section{CLETHRACEAE}

Clethra pringlei S. Wats.

23284AM 23821AM

\section{COMMELINACEAE}

Callisia hintoniorum B. L. Turner

Callisia navicularis (Ortgies) Hunt

Commelina dianthifolia Del.

Commelina diffusa Burm. $\mathrm{f}$.

Commelina elliptica Kunth

Commelina erecta L.

Gibasis gypsophila B. L. Turner

Gibasis hintoniorum B. L. Turner

Gibasis karwinskyana ssp. palmeri Hunt

Gibasis venustula (Kunth) Hunt Tinantia pringlei (S. Wats.) Rohw.

Tradescantia brachyphylla Greenm.

Tradescantia crassifolia Cav.

Tradescantia hirta Hunt

Tradescantia nuevoleonensis Matuda Tradescantia pringlei (S. Wats.) Rohw. Tradescantia sillamontana Matuda
22568ZR 23112ZR TYPE 23748AM 23848AM

18284GL 18492GL 19646GL 20409AT

20718AT 21124GL

22888AM 23315ZR

20551AM 21020GL 21430IT 22261ZR

23391ZR 23485ZR

22217ZR 22226ZR 22883AM 23174AM

23206AM 23225ZR

17959IT 20776RY 21104GL 22377GL

23172AM 23720AM 23747AM

22082GL TYPE 22376GL 23406ZR

23482ZR 23758AM

23013AM TYPE

17557ZR 18991AM 19612GL 19758GL

20811RY 21023GL 21286GL 21453IT

22140ZR 22271GL 22886AM 22915AM

23076AM 23386GL 23718AM

21055RA 21056RA

17592IT 17646ZR 21349GL 22597LN

$18457 \mathrm{GL}$

17416ZR

18276GL 18494GL 19647GL 20297AT

20410AT 21478IT 21560GL 23385GL

23513ZR 23605AM 23627AM 23694AM

18972AM 21105GL 21113GL

24109ST 24231MM

20785RY

\section{CONVOLVULACEAE}

Convolvulus arvensis L.

17138GL $19421 \mathrm{GL}$ 
Hinton \& Hinton: Hintons' Collections from South-Central Nuevo León and Adjacent Coahuila

Convolvulus equitans Benth.

Dichondra argentea $\mathrm{H}$. \& B.

Evolvulus alsinoides (L.) L.

Evolvulus rotundifolius (S. Wats.) Hallier

Evolvulus sericeus Sw.

Ipomoea cardiophylla A. Gray

Ipomoea carnea Jacq.

Ipomoea cordato-lobata Dennst.

Ipomoea cristulata Hall.

Ipomoea jalapa (L.) Pursh

Ipomoea miquihuanensis McDonald

Ipomoea purpurea (L.) Roth

Ipomoea rupicola House

Ipomoea trifida (Kunth) G. Don

Ipomoea tyrianthina Lindl.

Merremia dissecta (Jacq.) Hall. f.
20529AM 21926GL 22010AM 24214MM

24427ST

17611IT 18981AM 19696GL 23956AM

17528AM 17672IT 21116GL 21152SH

24268SV

20834RY 23404ZR

22986ZR

21674IT 24004AM

17952IT

20526AM

17674IT

17942IT 20363AM 21456IT 24198MM

$19261 \mathrm{GL} 19267 \mathrm{GL}$

20474GL 20752GL 23483ZR

24248SV

17612IT 21558IT

17645ZR 17657IT 17667GL 22294GL

22457ZR 22499ZR 22804AM 23276AM

23668AM

24279SH

CORNACEAE

Cornus disciflora DC.

22528ZR 22617ZR 22649AM 22688ZR

22742AM

22928AM

17054GL 18427AT 19455GL 22950ZR

Cornus excelsa Kunth
Cornus stolonifera Michx.

21321GL 21640GL 22744ZR

Cornus urbiniana Rose

CRASSULACEAE

Echeveria secunda Booth

17342ZR

19565GL

Echeveria simulans Rose

19998AM

Kalanchoë verticillata Ell.

Sedum calcicola Rob. \& Greenm.

18994AM 19215GL

Sedum gypsophilum B. L. Turner

18076GL

Sedum palmeri S. Wats.

17806ZR 19116GL 19287RY 20190RY

$20221 \mathrm{GL}$

Sedum parvum Hemsl.

17303GL 17551ZR 17919AT 17977AT

18310AT

Sedum wrightii A. Gray

17483ZR 18322GL 18588GL 19819GL

19890GL

$19624 \mathrm{GL}$

Sedum sp.

Villadia aristata Clausen

17261GL 17262GL 17975AT

Villadia cucullata Rose

Villadia jimulcensis Nesom

18929AT

18594GL

Villadia sp.

$19858 \mathrm{GL}$ 
Acta Botánica Mexicana (1995), 30:41-112

\section{CROSSOSOMATACEAE}

Forsellesia spinescens (A. Gray) Greene 18167GL 18706GL 22609GL

\section{CUCURBITACEAE}

Citrullus lanatus (Thunb.) Mats. \& Nakai

17727IT

Cucurbita foetidissima Kunth

Cucurbita texana (Scheele) A. Gray

Cyclanthera dissecta (T. \& G.) Arn.

18975GL 19510GL

Cyclanthera langaei Cogn.

Cyclanthera sp.

Lagenaria siceraria (Molina) Standl.

Melothria pendula L.

21443IT 21459IT

19246GL 20838RY 23472ZR

22921AM 23221ZR 23242ZR 23309ZR

23432ZR

20524AM

Sicyos laciniatus L.

20568AM 21212IT 21552IT

19701GL 19775GL

\section{CUPRESSACEAE}

Cupressus arizonica Greene

Juniperus deppeana Steud. var. deppeana

Juniperus erythrocarpa Cory

18948AM 20311AT 23319ZR

17816GL 20198AT 20498AT

Juniperus flaccida Schlecht.

17854AM

19899GL 20197RY 21194IT 21547IT

Juniperus monosperma (Engelm.) Sarg.

21623GL 22763ZR

Juniperus monticola Martinez

Juniperus pinchotii Sudw.

Juniperus saltillensis Hall.

21983AM 22047GL

20201AT

19103AM

18102GL 19706GL

CUSCUTACEAE

Cuscuta applanata Engelm.

Cuscuta glabrior (Engelm.) Yunck.

Cuscuta jalapensis Schlecht.

Cuscuta mitriformis Engelm.

23503ZR

20755GL

$21213 I T$

17631ZR 20029AM

CYCADACEAE

Dioon edule Lindl.

$17712 \mathrm{IT}$

CYPERACEAE

Carex planostachys Kunze

$20216 G L$

Carex schiedeana Kunze

18136GL 19661GL 20230AT 21069RA

Carex spissa Bailey

Carex ultra Bailey

21828GL 22717GL 23903AM

$18239 \mathrm{GL}$

21860GL 22723GL 
Hinton \& Hinton: Hintons' Collections from South-Central Nuevo León and Adjacent Coahuila

Cladium jamaicense Crantz

Cyperus canus Presl

Cyperus manimae Kunth

Cyperus pallidicolor (Kukenth.) Tucker

Cyperus thyrsiflorus Jungh.

Dichromena colorata (L.) Hitchc.

Eleocharis geniculata (L.) R. \& S.

Eleocharis montevidensis Kunth

Fuirena simplex Vahl var. simplex

Schoenus nigricans $\mathrm{L}$.

Scirpus analecti Beetle

Scirpus californicus (C. Mey.) Steud.

Scleria oligantha Michx.

Dioscorea urceolata Uline

DIOSCOREACEAE

Diospyros palmeri Eastw.

Ephedra aspera Engelm.

Ephedra pedunculata Engelm.

Equisetum hyemale L.

Arbutus xalapensis Kunth

Arctostaphylos pungens Kunth

Comarostaphylis polifolia ssp. minor (Small) Diggs

Lyonia squamulosa Mart. \& Gal.

Vaccinium kunthianum Klotz.
EBENACEAE

\author{
17903AM 21910GL \\ 19940AM 20917AM \\ 21127GL 23715AM \\ 22049GL 22115ZR 22506ZR 22922AM \\ 23092AM 23454ZR \\ 21407IT 21462IT 22216ZR 23719AM \\ 17853AM 20346AM 20405AM 23817ZR \\ 20326AM 21483IT \\ $21881 \mathrm{GL}$ \\ 20169RY \\ 21877GL 22034GL \\ 19231GL 21875GL 22037GL 23337GL \\ 24238GL \\ 21955AM \\ 23486ZR
}

17406ZR

EPHEDRACEAE

17738IT 24207MM

18508GL 19672GL

17905AM

EQUISETACEAE

18772GL 21856GL

ERICACEAE

17028GL 17377ZR 18161AT 19374GL 24085AM

17010GL 18204AT 18270AT 18375GL 20109GL 21793AT 21801GL 21834GL 22639DA

17344ZR 18217GL 18412GL 18417GL 18968AM 19377GL 22038GL 22793GL 23955AM

22933ZR 22991ZR 24030AM

17543ZR 22680ZR 22818AM 22907AM

22963ZR 24077AM 
EUPHORBIACEAE

Acalypha dioica S. Wats.

Acalypha lindheimeri Muell. Arg.

Acalypha monocephala Cav.

Acalypha monostachya Cav.

Acalypha lindheimeri Muell. Arg.

Acalypha phleoides Cav.

Acalypha sp.

Argythamnia astroplethes J. Ingram

Bernardia myricifolia (Scheele) Wats. var. myricifolia

Chamaesyce cinerascens (Engelm.) Small

Chamaesyce cumbrae (Boiss.) Millsp.

Chamaesyce fruticulosa (Engelm. ex Boiss.) Millsp.

Chamaesyce mendezii (Boiss.) Millsp.

Chamaesyce nutans (Lag.) Small

Chamaesyce serpyllifolia (Pers.) Small

Chamaesyce stictospora (Engelm.) Small var. stictospora

Chamaesyce villifera (Scheele) Small

Chiropetalum schiedeanum (Muell. Arg.) Pax \& Hoffm. Cnidoscolus rotundifolius (Muell. Arg.) McVaugh

Croton argyranthemus Michx.

Croton ciliatoglandulifer Ort.

Croton dioicus Cav.

Croton fruticulosus Torrey

Croton hypoleucus Schlecht.

Croton incanus Kunth

Croton monanthogynus Michx.

Croton suaveolens Torrey

Croton virletianus Muell. Arg.

Euphorbia antisyphilitica Zucc.

Euphorbia beamanii M. C. Johnston

Euphorbia bifurcata Engelm.

Euphorbia brachycera var. greggii (Boiss.) M. C. Johnston

Euphorbia furcillata Kunth var. furcillata

Euphorbia furcillata var. ribana M. C. Johnston Euphorbia graminea Jacq.

Euphorbia longecornuta S. Wats.

Euphorbia macropus (KI. \& Gke.) Boiss.

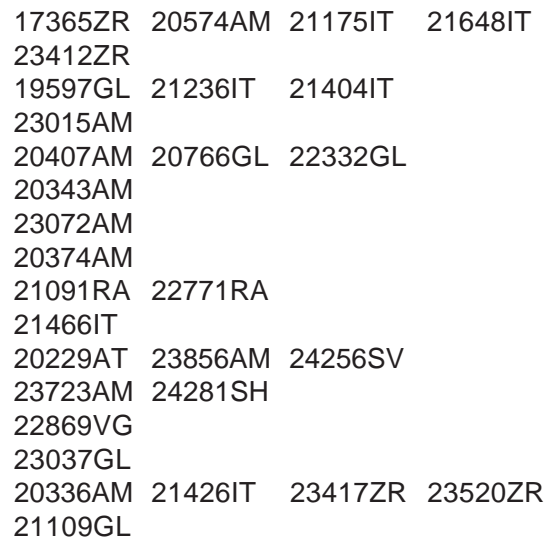


Hinton \& Hinton: Hintons' Collections from South-Central Nuevo León and Adjacent Coahuila

Euphorbia macvaughiana M. C. Johnston Euphorbia monterreyana Millsp.

Euphorbia neilmulleri M. C. Johnston

\section{Euphorbia sp.}

Jatropha dioica Cav.

Manihot subspicata Rogers \& Appan

Phyllanthus carolinensis Walt.

Phyllanthus polygonoides Spreng.

Poinsettia cyathophora (Murr.) KI. \& Gke.

Poinsettia dentata (Michx.) KI. \& Gke.

Poinsettia tubadenia Mayfield (ined.)

Tragia nepetifolia Cav.

\author{
20106GL \\ 22896AM \\ 18170GL 18387GL 20189RY 20948GL \\ 21861GL 22095GL 22699AM 23516ZR \\ 23641ZR 24243GL \\ 18959AM 21388IT 21451IT 23332GL \\ 23518ZR 23898AM 24245GL \\ 20921AM 22790RA \\ 24255SV \\ 20559AM \\ 23849AM \\ 24276SH \\ 21200IT 23393ZR 23396ZR 23475ZR \\ 24830GL TYPE 19493GL 19566GL \\ 21557IT 23521ZR 23672AM 23902AM \\ 18969AM
}

\section{FABACEAE}

Acacia angustisissima (Mill.) O. Ktze.

Acacia berlandieri Benth.

Acacia constricta Benth.

Acacia coulteri Benth.

Acacia farnesiana (L.) Willd.

Acacia rigidula Benth.

Acacia roemeriana Scheele

Amicia zygomeris DC.

Astragalus coriaceus Hemsl.

Astragalus diphactus S. Wats.

Astragalus emoryanus (Rydb.) Cory

Astragalus greggii S. Wats.

Astragalus hypoleucus Schauer

Astragalus legionensis Barneby

Astragalus mollissimus var. irolanus (Jones) Barneby

Astragalus nuttallianus var. trichocarpus T. \& G.

Astragalus parvus Hemsl.

Astragalus purpusii Jones

Astragalus racemosus Pursh

Astragalus sanguineus Rydb.

Bauhinia macranthera Benth. ex Hemsl.

Bauhinia ramosissima Benth. ex Hemsl.

Bauhinia uniflora S. Wats.

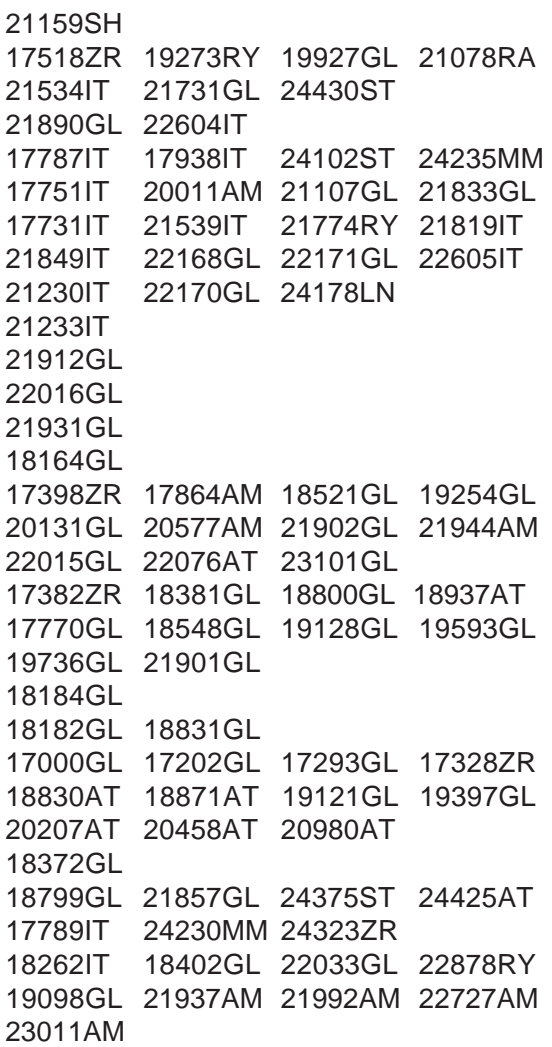

21159SH

17518ZR 19273RY 19927GL 21078RA

21534IT 21731GL 24430ST

21890GL 22604IT

17787IT 17938IT 24102ST 24235MM

17751IT 20011AM 21107GL 21833GL

17731IT 21539IT 21774RY 21819IT

21849IT 22168GL 22171GL 22605IT

21230IT 22170GL 24178LN

$21233 I T$

$21912 \mathrm{GL}$

22016GL

$21931 \mathrm{GL}$

18164GL

17398ZR 17864AM 18521GL 19254GL

20131GL 20577AM 21902GL 21944AM

22015GL 22076AT 23101GL

17382ZR 18381GL 18800GL 18937AT

17770GL 18548GL 19128GL 19593GL

19736GL 21901GL

$18184 \mathrm{GL}$

$18182 \mathrm{GL} 18831 \mathrm{GL}$

17000GL 17202GL 17293GL 17328ZR

18830AT 18871AT 19121GL 19397GL

20207AT 20458AT 20980AT

$18372 \mathrm{GL}$

18799GL 21857GL 24375ST 24425AT

17789IT 24230MM 24323ZR

18262IT 18402GL 22033GL 22878RY

19098GL 21937AM 21992AM 22727AM

23011AM 
Brongniartia foliosa Benth.

Brongniartia intermedia Moric.

Caesalpinia atropunctata Eiffert

Caesalpinia gilliesii Wall.

Caesalpinia mexicana A. Gray

Calliandra conferta Benth.

Calliandra eriophylla Benth.

Canavallia septentrionalis Sauer

Cassia vogeliana Schl.

Centrosema virginianum (L.) Benth.

Cercidium macrum I. M. Johnston

Cercis canadensis $\mathrm{L}$.

Chamaecrista greggii (A. Gray) Pollard ex Heller

Chamaecrista lindheimeri (Schiede) Irwin \& Barneby

Cologania angustifolia Kunth

Cologania broussonetii (Balb.) DC.

Crotalaria sagittalis L.

Dalea bicolor var. argyrea (A. Gray) Barneby

Dalea bicolor H. \& B. ex Willd. var. bicolor

Dalea botterii (Rydb.) Barneby var. botterii

Dalea brachystachya A. Gray

Dalea capitata S. Wats. var. capitata

Dalea capitata var. lupinocalyx Barneby

Dalea capitata var. pseudo-hospes Barneby

Dalea eriophylla S. Wats. var. eriophylla

Dalea filiciformis B. Rob. \& Greenm.

Dalea greggii A. Gray

Dalea gypsophila Barneby

Dalea hospes (Rose) Bullock

Dalea lutea (Cav.) Willd.

Dalea melantha var. berlandieri (A. Gray) Barneby

Dalea melantha Schauer var. melantha

Dalea obovatifolia Ort. var. obovatifolia

Dalea pogonathera A. Gray

Dalea prostrata Ort.

Dalea radicans $\mathrm{S}$. Wats.

Dalea staffordii (Rose) Bullock

Dalea uniflora (Barneby) Nesom

Dalea wrightii A. Gray

Desmanthus painteri (Britt. \& Rose) Standl.

Desmanthus pringlei (Britt. \& Rose) Hermann

Desmodium caripense (Kunth) G. Don

\author{
21267GL 22941ZR 23474ZR \\ 20369AM 22735AM 22807AM \\ 22877DA \\ 17533AM \\ 17624IT 17744IT 17785IT 21225IT \\ 22183SC \\ 21423IT 21498IT 21544IT 23063AM \\ 17654IT 19714GL 21024GL 22028GL \\ 23854AM \\ 17637ZR 22145ZR 24316ZR \\ 17397DA \\ 17677LN \\ 24176AN \\ 17051GL 17517ZR 19353GL 20133GL \\ 22756ZR \\ 17937IT 20922AM 21229IT 23872AM \\ 24261SV \\ 21181IT \\ 19153GL 21040GL 22847AM \\ 17616IT 21277GL \\ 22587ZR 22619ZR 23231ZR \\ 23776AM \\ 19763GL 20623DA 20633DA \\ 17437ZR 22454ZR 23299ZR 23941AM \\ 20611DA \\ 21387IT \\ $17183 \mathrm{GL}$ \\ 17444DA 18433GL 19705GL 19738GL \\ 17313GL 18114GL 18781GL 19091AM \\ 19314ZR \\ 18169GL 20931AM 22696AM 23746AM \\ 23857AM 23992AM \\ 18382GL 19111AM 19284RY 20847RY \\ 21755GL 22027GL 23845AM \\ $18231 \mathrm{GL} 19527 \mathrm{GL}$ \\ $18711 \mathrm{GL}$ \\ 17632ZR 18280GL 18989AM 21335GL \\ 23506ZR \\ 21991AM 22779RA \\ 17289GL 18383GL 19317ZR 19821GL \\ 23762AM \\ 22872VG \\ 19079AM \\ 20226AT \\ 17757GL 19299GL 19359AT 20110GL \\ 21798GL 21900GL 22715DA 22726GL \\ 18752GL 18961AM 21966AM 21971AM \\ $21146 \mathrm{SH}$ \\ 18996AM \\ 24225MM \\ 20552AM 21436IT 23425ZR 23831AM
}


Hinton \& Hinton: Hintons' Collections from South-Central Nuevo León and Adjacent Coahuila

Desmodium glutinosum (Muhl. ex Willd.) Wood

Desmodium grahamii A. Gray

Desmodium lindheimeri Vail

Desmodium psilophyllum Schlecht.

Desmodium retinens Schlecht.

Desmodium subrosum Nesom

Desmodium tortuosum (Sw.) DC.

Erythrina herbacea L.

Eysenhardtia parvifolia Brandeg.

Eysenhardtia polystachya (Ort.) Sarg.

Eysenhardtia texana Scheele

Galactia wrightii A. Gray

Genistidium dumosum I. M. Johnston

Hoffmanseggia glauca (Ort.) Eifert

Hoffmanseggia oxycarpa A. Gray

Hoffmanseggia watsonii (Fisher) Rose

Indigofera lindheimeriana Scheele

Indigofera miniata Ort.

Indigofera suffruticosa Mill.

Lathyrus parvifolius S. Wats.

Leucaena greggii S. Wats.

Lotus hintoniorum B. L. Turner

Lupinus cacuminis Standl.

Lupinus hintoniorum B. L. Turner

Lupinus partisans Rose

Lupinus platamodes C. P. Smith

Lupinus potosinus Rose

Lupinus stipulatus var. exilis C. P. Smith

Lupinus sp.

Macroptilium longepedunculatum (Mart. ex Benth.) Urb. 17735IT

Medicago lupulina L.

Medicago sp.

Melilotus albus L.

Melilotus indicus (L.) Ait.

Mimosa aculeaticarpa Ort.

Mimosa biuncifera Benth.

Mimosa malacophylla A. Gray

Mimosa potosina (Britt. \& Rose) B. L. Turner

Mimosa texana (A. Gray) Small

Mimosa zygophylla Benth.

Nissolia platycalyx S. Wats.

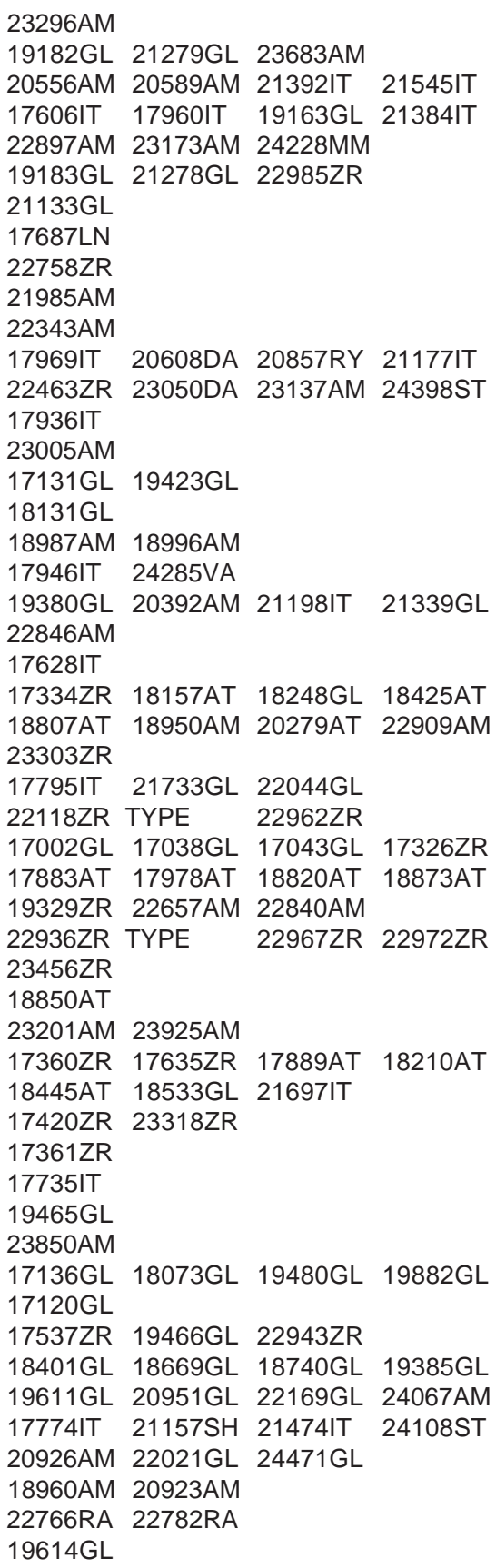


Orbexilum melanocarpum (Benth. ex Hemsl.) Rydbg. Oxyrhynchus populneus Piper

Peteria glandulosa A. Gray

Phaseolus coccineus L. ssp. coccineus

Phaseolus leptostachys Benth.

Phaseolus neglectus Hermann

Phaseolus pedicellatus Benth.

Phaseolus polymorphus S. Wats.

Phaseolus sp.

Pithecellobium elastichophyllum A. Gray

Pithecellobium leptophyllum (Cav.) Daveau

Pithecellobium pallens (Benth.) Standl.

Prosopis glandulosa Torrey var. glandulosa

Prosopis glandulosa var. torreyana (Benson) M. C. Johnston

Psoralea melanocarpa Benth.

Rhynchosia longiracemosa Mart. \& Gal.

Senna bauhinioides (A. Gray) Irwin \& Barneby

Senna crotalarioides (Kunth) Irwin \& Barneby

Senna lindheimeriana (Scheele) Irwin \& Barneby

Senna aff. lindheimeriana (Scheele) Irwin \& Barneby

Senna occidentalis (L.) Irwin \& Barneby

Senna wislizeni var. painteri (Britt.) Irwin \& Barneby

Sophora juanhintoniana B. L. Turner

Sophora nuttalliana B. L. Turner

Sophora secundiflora (Ort.) DC.

Stylosanthes viscosa Sw.

Trifolium aff. dubium Sibth.

Trifolium mexicanum Hemsl.

Vicia humilis Kunth

Vicia ludoviciana Nutt. var. Iudoviciana

Vicia pulchella Kunth
19144GL

21235IT

23109ZR 23703AM

22235ZR 23477ZR

17697LN 21391IT 22564ZR

19177GL 21258GL 23816ZR

22479ZR 23241ZR 23300ZR 23304ZR

17245GL 23421ZR 23424ZR

23251ZR

$21832 \mathrm{GL}$

19239GL 19378GL 19379GL 20813RY

21394IT 22175GL

17950IT 21422IT 21578GL 24442ST

24451RY

19664GL 22174GL 22791RA

24071AM

18539GL 18684GL

17692LN

19127GL 19592GL

18965AM 23100GL

19893GL 20066AM 22189SC 23787AM

$17625 \mathrm{IT}$

17538AM 22884AM

17810ZR 17906AM 21118GL 21825IT

22265ZR 22323GL

23025AM 24053AM TYPE

$18414 \mathrm{GL}$

18966AM 19319ZR 19383GL 19648GL

21987AM

21471IT

18792GL

22213ZR 22923AM 23096AM 23422ZR

17005GL 18162AT 18208AT 18314AT

19117GL 20985AT 22233ZR 24021AM

24049GL

18435GL 18527GL

17041GL 18804AT 19150GL 19400GL 20271AT 23306ZR

\section{FAGACEAE}

Quercus affinis Scheidw.

Quercus canbyi Trel.

Quercus coccolobifolia Trel. Quercus crassifolia H. \& B. Quercus crassipes H. \& B. Quercus diversifolia Née
17267GL 18338AT

17286GL 17717IT 17720IT 17811ZR

18395GL 18397GL 20179RY 21195IT

22982ZR 22989ZR 23409ZR 24459GL

22675AM 22676AM

23931AM 23935AM

17435ZR

19255GL 19256GL 22558ZR 
Hinton \& Hinton: Hintons' Collections from South-Central Nuevo León and Adjacent Coahuila

Quercus durifolia v. Seem.

Quercus eduardii Trel.

Quercus endlichiana Trel.

Quercus flocculenta C. H. Muller

Quercus fusiformis Small

Quercus galeanensis C. H. Muller

Quercus glaucoides Mart. \& Gal.

Quercus greggii (DC.) Trel.

Quercus aff. hartmannii Trel.

Quercus hintoniorum Nixon \& C. H. Muller

Quercus hypoxantha Trel.

Quercus intricata Trel.

Quercus invaginata Trel.

Quercus laeta Liebm.

Quercus laurina H. \& B.

Quercus mexicana H. \& B.

Quercus microphylla Née

Quercus miquihuanensis Nixon \& C. H. Muller Quercus omissa DC.

Quercus opaca Trel.

Quercus polymorpha Schlecht. \& Cham.

Quercus pringlei v. Seem.

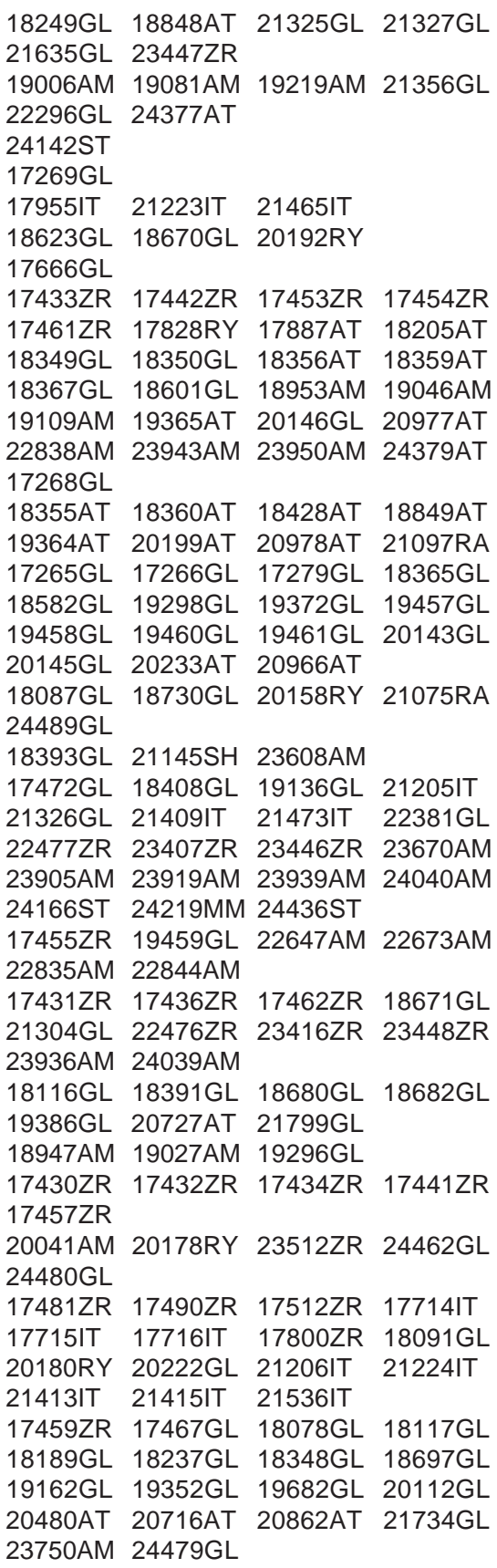


Quercus rysophylla Weath.

22624ZR 22992AM 23295AM 23826AM

Quercus saltillensis Trel.

24101ST

Quercus sideroxyla H. \& B.

17578ZR 18374GL

Quercus striatula Trel.

17456ZR 20176RY 22979ZR 23408ZR

Quercus sp.

$19297 \mathrm{GL}$

17458ZR 17460ZR 19007AM 20135GL

\section{FLACOURTIACEAE}

Neopringlea integrifolia (Hemsl.) S. Wats.

19996AM 20294AT 21549IT 21770AM

Xylosma flexuosum (Kunth) Hemsl.

22734AM 23679AM 23996AM

17589IT 17743IT 21550IT

\section{FRANKENIACEAE}

Frankenia gypsophila I. M. Johnston

18224GL 19535GL 19563GL 19643GL 23028GL

FUMARIACEAE

Corydalis pseudomicrantha Fedde

17985AT 18159AT 18812AT 20286AT
22601LN

\section{GARRYACEAE}

Garrya glaberrima Wang.

18687GL 18773GL

Garrya laurifolia ssp. macrophylla (Benth.) Dahling

17281GL 17500ZR 21358GL 22426ZR 22642ZR

Garrya ovata Benth. ssp. ovata

17466GL 17886AT 18735GL 20139GL 20204GL 22405ZR

\section{GENTIANACEAE}

Centaurium calycosum (Buckl.) Fern. var. calycosum

Centaurium chironioides (Griseb.) Druce

Centaurium tenuifolium (Mart. \& Gal.) Rob.

Eustoma exaltatum (L.) G. Don

Geniostemon gypsophilum B. L. Turner

Gentiana spathacea Kunth

Gentianella amarella ssp. acuta (Michx.) Herder.

Halenia brevicornis var. multiflora Allen

Halenia plantaginea (Kunth) Griseb.

Halenia recurva (Smith) Allen

Sabatia stellaris Pursh

Swertia radiata (Kell.) O. Ktze.

17857AM 18607GL 20909AM 21737GL 22080GL 22313GL

18279GL 21882GL 22042GL

17570ZR

17830RY 18273RY 20327AM

22354AM TYPE

23940AM

$17244 \mathrm{GL}$

18342AT

22223ZR 23426ZR

20450AT

$17901 \mathrm{AM}$

17299GL 
Hinton \& Hinton: Hintons' Collections from South-Central Nuevo León and Adjacent Coahuila

\section{GERANIACEAE}

Erodium cicutarium (L.) L'Hér.

17022GL 17764GL 18545GL 19362AT

Geranium crenatifolium H. E. Moore

19484GL 20105GL 24022AM

17056GL 17114GL 17335ZR 17873AT

18821AT 20283AT 20984AT 20990AT

21000AT 24385AT

Geranium potosinum Moore

$17013 \mathrm{GL}$

Geranium seemannii Peyr.

17007GL 17874AT 19550GL 20453AT

21320GL 21622GL 22206ZR 22842AM 22956ZR

Geranium tenue Hanks

20993AT

\section{GESNERIACEAE}

Achimenes antirrhina (DC.) Morton

17401ZR

HAMAMELIDACEAE

Liquidambar styraciflua L.

22627ZR

HYDROPHYLLACEAE

Nama biflorum Choisy

Nama canescens Hitchc.

Nama dichotomum (Ruiz \& Pavón) Choisy

Nama hintoniorum Nesom

Nama hispidum A. Gray

Nama hitchcockii Bacon

Nama palmeri A. Gray

Nama sericeum R. \& S. ex Willd.

Nama stevensii var. gypsicola (I. M. Johnston) Bacon

Nama stewartii I. M. Johnston

Nama undulatum Kunth

Nemophila patuliflora var. teucriifolia (Johnston)

Phacelia congesta Hook.

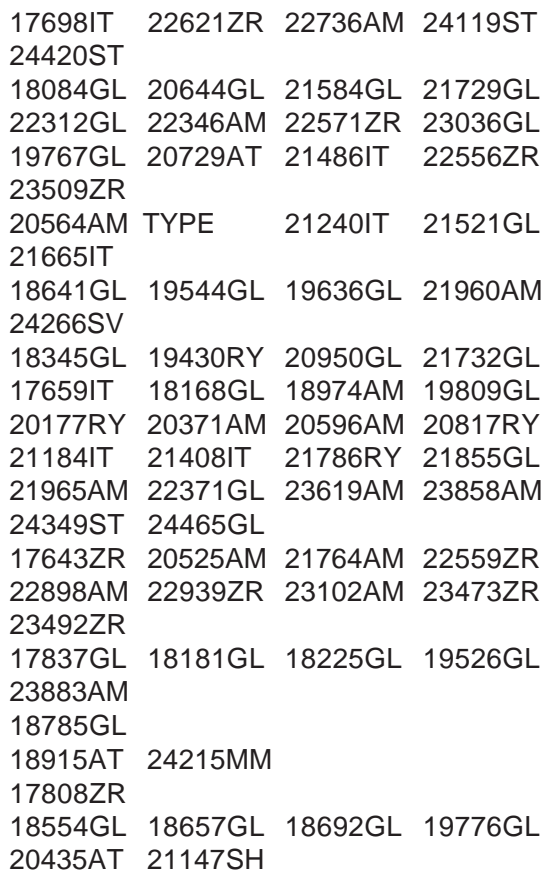
23509ZR 
Phacelia gypsogenia I. M. Johnston

Phacelia hintoniorum B. L. Turner

Phacelia patuliflora var. teucrifolia (I. M. Johnston) Constance

Phacelia pinnata (R. \& P.) Macbr.

Phacelia platycarpa (Cav.) Spreng.

Phacelia potosina B. L. Turner

Phacelia robusta (Macbr.) I. M. Johnston

Phacelia rupestris Greene

Phacelia vossii Atwood

Phacelia zaragozana B. L. Turner
18185GL 20280GL 22314GL

18223GL TYPE

17036GL 18851AT

17119GL 17885AT 18884AT

17366ZR 18311AT

18453GL TYPE

21046RA

19140GL 20800RY 21017GL 22560ZR

18327GL 19222GL 21967AM 22574ZR

23121ZR 23730AM

17868ZR TYPE 22439ZR 23322ZR

HYPERICACEAE

Hypericum formosum Kunth

Hypericum pauciflorum Kunth
22292GL 23150AM

IRIDACEAE

Iris missouriensis Nutt.

Sisyrinchium arizonicum Rothr.

Sisyrinchium convolutum Nocca

Sisyrinchium dimorphum Oliver

Sisyrinchium hintoniorum Nesom

Sisyrinchium micranthum Cav.

Sisyrinchium microbracteatum Nesom

Sisyrinchium novoleonense Nesom \& Hernández

Sisyrinchium scabrum Cham. \& Schlecht.

Sisyrinchium schaffneri S. Wats.

Tigridia multiflora (Baker) Ravenna

\author{
17888AT 18209AT \\ 20420AT \\ $18536 \mathrm{GL}$ \\ 24133ST 17859GL \\ 22440ZR TYPE \\ 21946AM \\ 21570GL 23104ZR 23737AM TYPE \\ 17298GL 17368ZR 23098AM \\ 18295AT 18584GL 19799GL 20281AT \\ 21928GL 22180GL 24504GL \\ 17116GL 18040AM 18816AT 18963AM \\ 20276AT 21845GL 22610ZR 22725GL \\ 22954ZR 24080AM \\ 23086AM 23224ZR
}

\section{JUGLANDACEAE}

Carya illinoinensis (Wang.) K. Koch

Carya myristiciformis (Michx. f.) Nutt.

Carya ovata var. mexicana (Engelm.) Manning

Carya palmeri Manning

Juglans hirsuta Manning

Juglans mollis Hemsl.
18396GL 19241GL

21374GL

23442ZR

24321ZR

22167GL

23418ZR

\section{JUNCACEAE}

Juncus arcticus var. neomexicanus (Willd.) Balslev 22797GL

Juncus texanus (Engelm.) Cov.

22065GL 
Hinton \& Hinton: Hintons' Collections from South-Central Nuevo León and Adjacent Coahuila KOEBERLINIACEAE

Koeberlinia spinosa Zucc.

17844GL 17866AM 20082GL 20600DA 20628DA

\section{KRAMERIACEAE}

Krameria cytisoides Cav.

Krameria grayi Rose \& Painter

Krameria parviflora DC.

Krameria ramosissima (A. Gray) S. Wats.
17285GL 18694GL 19283ZR 19613GL 20963AT 24063AM

23012AM

19583GL

24249SV

\section{LAMIACEAE}

Agastache pallidiflora var. havardii (A. Gray) Sanders Agastache palmeri var. galeanum B. L. Turner Agastache palmeri var. leonensis Sanders

Hedeoma ciliolatum (Epl. \& Stew.) Irving

Hedeoma aff. ciliolatum (Epl. \& Stew.) Irving Hedeoma costatum A. Gray

Hedeoma drummondii Benth. var. drummondii

Hedeoma palmeri var. galeanum B. L. Turner Hedeoma palmeri Hemsl. var. palmeri

Hedeoma pusillum (Irving) Irving

Hedeoma quercetorum Epl.

Hyptis mutabilis (A. Rich.) Briq.

Leonotis nepetifolia $\mathrm{R}$. Br.

Lepechinia schiedeana (Schlecht.) Vatke

Marrubium vulgare L.

Monarda citriodora ssp. austromontana (Graham) Fern. 17125GL 17250GL

Monarda citriodora var. attenuata Scora

Monarda citriodora Cerv. var. citriodora

Monarda fistulosa var. menthifolia (Graham) Fern.

Monarda pringlei Fern.

Physostegia correllii (Lund.) Shinners

Poliomintha longiflora A. Gray

Prunella vulgaris L.

Salvia azurea Lam.

Salvia ballotaeflora Benth.

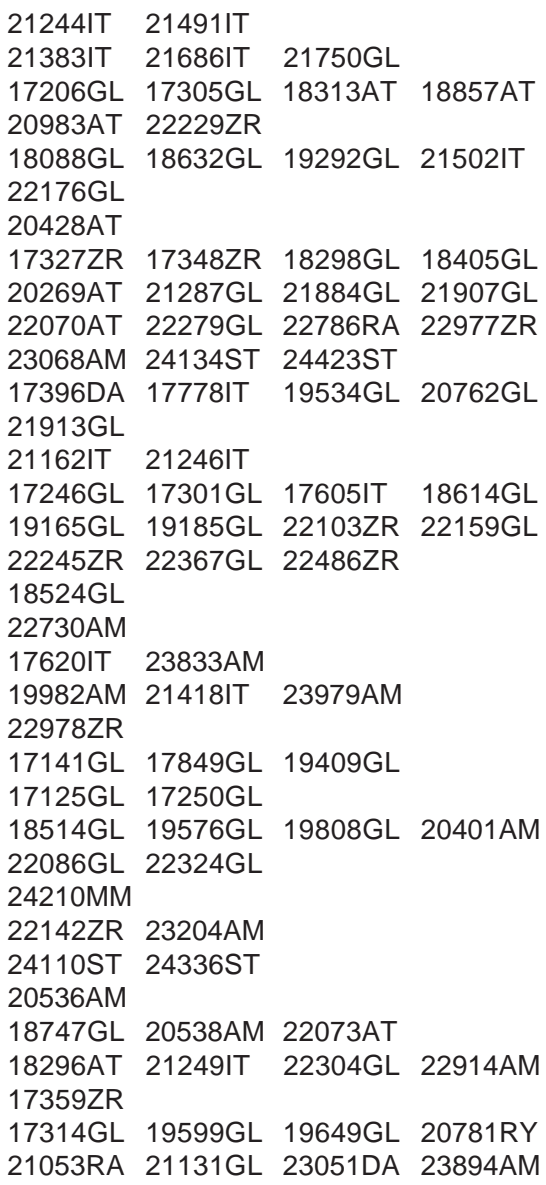


Salvia caudata Epl.

Salvia chamaedryoides Cav.

Salvia coahuilensis Fern.

Salvia coccinea Juss.

Salvia compsostachys Benth.

Salvia coulteri Epl.

Salvia forreri Greene

Salvia grahamii Benth.

Salvia greggii A. Gray

Salvia involucrata Cav.

Salvia keerlii Benth.

Salvia longistyla Benth.

Salvia lycioides A. Gray

Salvia macellaria Epl.

Salvia aff. macellaria Epl.

Salvia microphylla Kunth

Salvia modica Epl.

Salvia oresbia Fern.

Salvia prunelloides Benth.

Salvia puberula A. Gray

Salvia reflexa Hornem.

Salvia regla Cav.

Salvia riparia Kunth

Salvia roemeriana Scheele

Salvia rotundifolia (L.) Huds.

Salvia rubropunctata Robins. \& Fern.

Salvia sacculus Epl.

Salvia setulosa Fern.

Salvia tarayensis Peterson

Salvia tiliaefolia Vahl

Salvia unicostata Fern.

Salvia urolepis Fern.

Salvia aff. urolepis Fern.

Salvia villosa Fern.

Salvia sp. nov. Ramamoorthy (\#1)

Salvia sp. nov. Ramamoorthy (\#2)

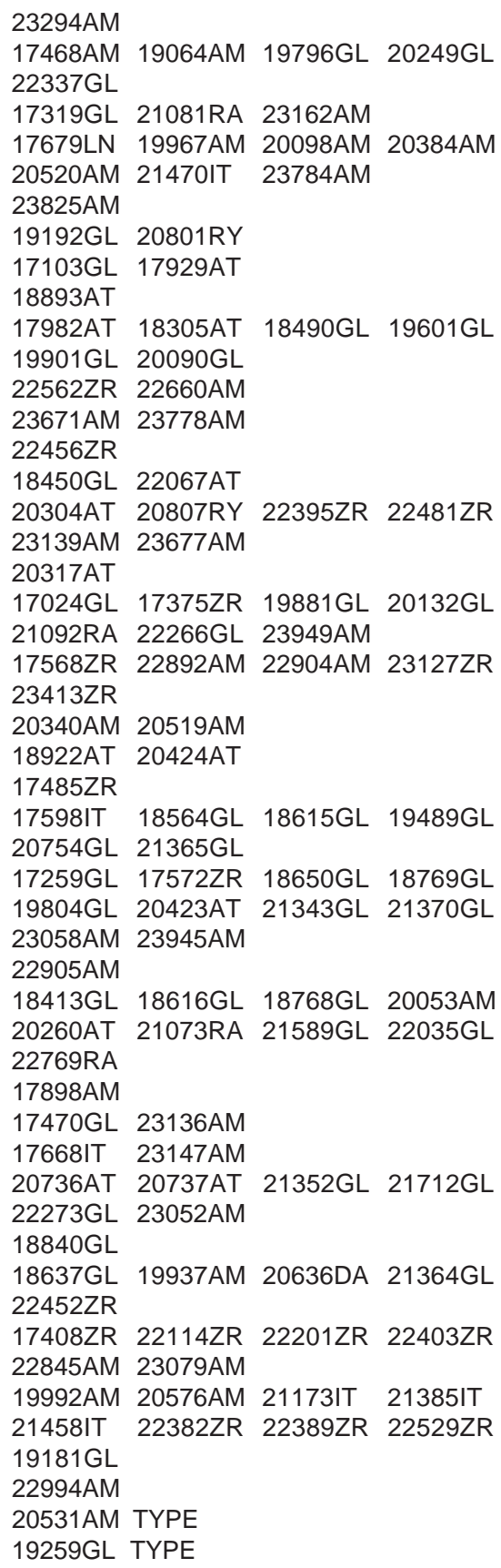


Hinton \& Hinton: Hintons' Collections from South-Central Nuevo León and Adjacent Coahuila

Salvia sp. nov. Ramamoorthy (\#3)

Salvia sp. nov. Ramamoorthy (\#4)

Salvia sp. nov. Ramamoorthy (\#5)

Salvia sp.

Satureja hintoniorum B. L. Turner

Scutellaria aramberrana B. L. Turner

Scutellaria bartlettii B. L. Turner

Scutellaria caerulea Moc. \& Sessé

Scutellaria drummondii Benth.

Scutellaria hintoniorum Henrickson

Scutellaria hispidula B. Rob.

Scutellaria lutilabia Lane \& Nesom

Scutellaria monterreyana B. L. Turner

Scutellaria potosina var. novoleonensis B. L. Turner

Scutellaria potosina Brandeg. var. potosina

Scutellaria seleriana Loes.

Scutellaria suffrutescens S. Wats.

Stachys agraria Cham. \& Schlecht.

Stachys bigelovii A. Gray

Stachys eriantha Benth.

Stachys grahamii Benth.

Stachys hintoniorum B. L. Turner

Stachys langmaniae Rzed. \& Rzed.

Stachys sp. nov. B. L. Turner

Teucrium canadense L.

Teucrium cubense Jacq. ssp. cubense

Teucrium cubense ssp. laevigatum (Vahl) McClint.

Teucrium vesicarium Mill.
Stachys vulnerabilis Rzed. \& Rzed.

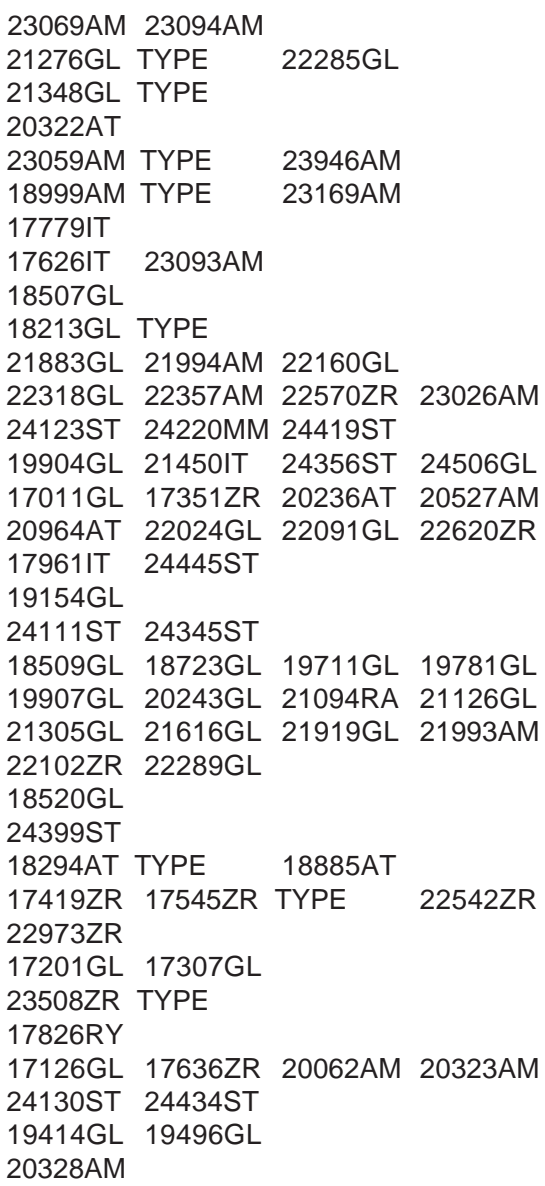

\section{LAURACEAE}

Litsea glaucescens Kunth

Litsea muelleri Rehd.

Persea pachypoda Nees

Phoebe tampicensis (Meiss.) Mez

17491ZR 17493ZR 21811GL 22630ZR

22640ZR 22728AM 22831AM

17280GL 18133GL 18384GL 19344GL 20182RY 21872GL

22632ZR

$17747 \mathrm{IT}$

\section{LEMNACEAE}

Lemna minuscula Hert.

18038AM 


\section{LENTIBULARIACEAE}

Pinguicula cyclosecta Casper

Pinguicula gracilis Zamudio Pinguicula hintoniorum B. L. Turner Pinguicula immaculata Zamudio \& Lux Pinguicula jorgehintonii B. L. Turner Pinguicula kondoi Casper Pinguicula reticulata Schlauer Pinguicula rotundiflora Studnicka

\section{LILIACEAE}

Allium glandulosum Link \& Otto Allium hintoniorum B. L. Turner

Allium kunthii G. Don

Allium sp.

Asphodelus fistulosus L.

Calochortus marcellae Nesom

Dasylirion berlandieri S. Wats.

Echeandia leptophylla Benth.

Echeandia mexicana Cruden

Echeandia sp.

Maianthemum scilloideum (M. \& G.) LaFrankie

Nolina caespitifera Trel.

Nolina nelsonii Rose

Nolina sp.

Nothoscordum bivalve (L.) Britt.

Schoenocaulon officinale (Schlecht. \& Cham.) Benth. Schoenocaulon texanum Scheele

Schoenocaulon aff. texanum Scheele

Schoenocaulon sp.

Smilacina stellata (L.) Desf.

Smilax bona-nox L.

Smilax lanceolata L.

Smilax moranensis Mart. \& Gal.

Smilax tamnoides L.

Yucca carnerosana (Trel.) McKelvey

Yucca decipiens Trel.

Yucca filifera Chabaud

Zigadenus hintoniorum B. L. Turner
17641ZR 22507ZR 22539ZR 22966ZR

23080AM 23125ZR 23240ZR 23257ZR

19349GL 21309GL 24044AT 24045GL

19312ZR 22661DA TYPE

19294GL 20191RY

24000AM TYPE

19021AM

21936AM 22590AM

21812GL

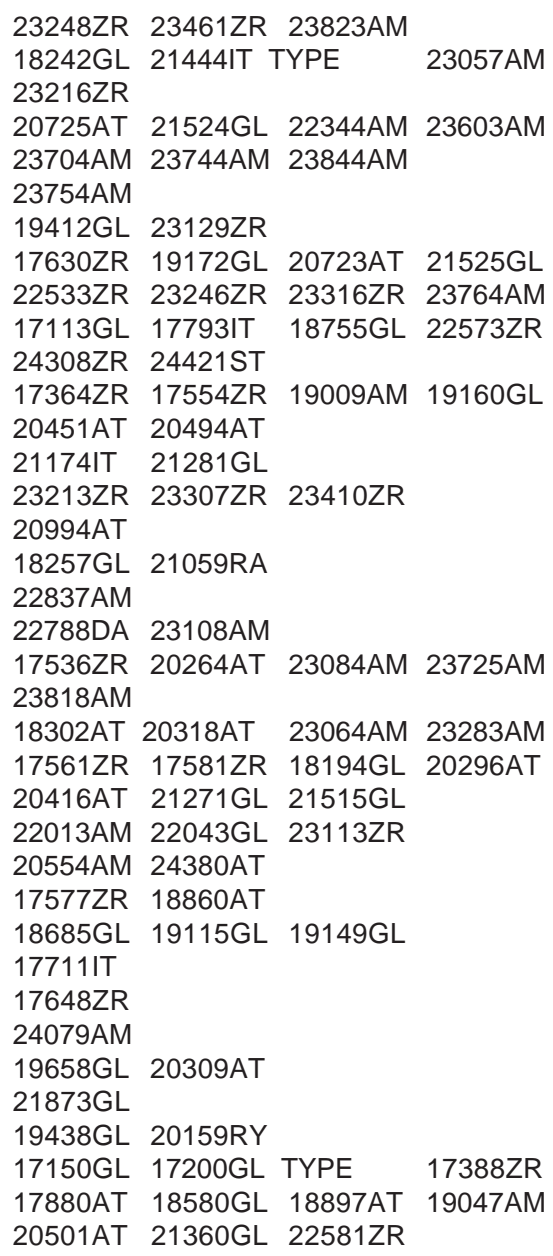


Hinton \& Hinton: Hintons' Collections from South-Central Nuevo León and Adjacent Coahuila

LINACEAE

Linum lasiocarpum Rose

18725GL 24232MM 24409ST

Linum lewisii Pursh

18846AT 20211AT 20476GL 20759GL 22400ZR

Linum modestum Rogers

Linum rupestre (A. Gray) Engelm.

18137GL 18196GL 18240GL 18759AM

19293GL 20155RY

18526GL 19311ZR 20411AT 21970AM

22322GL 22697AM 22708DA 23115ZR

23592AM 23743AM 24246GL 24254SV

Linum scabrellum Planch.

Linum schiedeanum Schlecht. \& Cham.

19106AM 22320GL 22707DA 23043GL

17562ZR 18971AM 19468GL 19692GL

21307GL 22270GL 22305GL

Linum vernale (A. Gray) Engelm.

17662IT

\section{LOASACEAE}

Cevallia sinuata Lag.

Eucnide lobata (Hook.) A. Gray

18095GL 18329GL 18456GL 19289RY

19341AM 19978AM 20935AM 22334GL

17821RY 18640GL 19274RY 19334ZR

19348GL 20930AM 21047RA 21208IT

21715GL 21835GL 22157SC 22764ZR

23103AM 23114ZR 23705AM 23752AM

24418ST

Eucnide xylinea C. H. Muller

Mentzelia asperula Woot. \& Standl.

Mentzelia hispida Willd.

24145ST 24365ST

23610AM

17655IT 18698GL 19194GL 19762GL

20772RY 21586GL 22561ZR 22569ZR

23336GL 23633ZR 23643ZR 23738AM

24213MM

21646IT

Mentzelia lindheimeri Urb. \& Gilg

21996AM

Mentzelia pachyrhiza I. M. Johnston

Mentzelia reverchonii (U. \& G.) T. \& Z.

$18301 \mathrm{GL} 19447 \mathrm{GL}$

\section{LOGANIACEAE}

Buddleja cordata ssp. tomentella (Standl.) Norman

Buddleja marrubiifolia Benth.

Buddleja scordioides Kunth

Buddleja sessilifolia Kunth
17186GL 17600IT 19214GL 19800GL 21086RA 21207IT 21397IT 21752GL 22412ZR 23756AM 23802ZR 24392ST $18077 \mathrm{GL} 19715 \mathrm{GL}$ 18559GL 19769GL $18141 \mathrm{IT}$

\section{LORANTHACEAE}

Arceuthobium campylopodum f. blumeri (A. Nels.) Gill 17273GL Arceuthobium douglasii Engelm.

$17271 \mathrm{GL}$

Arceuthobium vaginatum (Willd.) Presl

$17023 \mathrm{GL}$ 
Phoradendron bolleanum ssp. densum (Torr.) Wiens Phoradendron lanceolatum Engelm.

Phoradendron saltillense Trel.

Phoradendron schumannii Trel.

Phoradendron sp.
17904AM

17283GL 17758GL $19716 \mathrm{GL}$ 17363ZR 22048GL

LYTHRACEAE

Cuphea aequipetala Cav.

Cuphea cyanea DC.

Cuphea lanceolata Ait.

Heimia salicifolia (Kunth) Link \& Otto Lythrum californicum T. \& G.

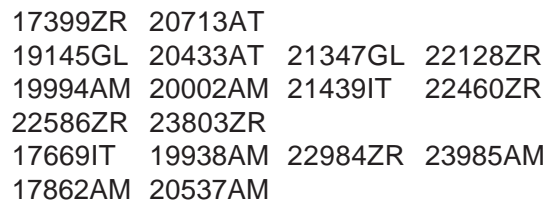

\section{MALPIGHIACEAE}

Aspicarpa hyssopifolia A. Gray

Callaeum macropterum (DC.) D. Johnson

Callaeum septentrionale (Juss.) D. Johnson

Janusia sp.

Malpighia glabra L.

Mascagnia lilacina (S. Wats.) Nied.

Thryallis glauca (Cav.) Kuntze
23622AM

$17590 I T$

17788IT 19432RY 21137SH 21228IT

21535IT 22187SC 24314ZR

20832RY

17678LN

20809RY 20844RY 21065RA 21156SH

24447ST

24313ZR

MALVACEAE

Abutilon ellipticum Schldl.

Abutilon hypoleucum A. Gray

Abutilon malacum S. Wats.

Abutilon wrightii A. Gray

Allowissadula holoserica (Scheele) Bates

Anoda aff. albiflora Fryx.

Anoda cristata (L.) Schlecht.

Batesimalva violacea (Rose) Fryxell

Callirhoe involucrata var. tenuissima Palmer ex Baker

Herissantia crispa (L.) Brizicky

Hibiscus acicularis Standl.

Hibiscus cardiophyllus A. Gray

Hibiscus coulteri A. Gray

Hibiscus martianus Zucc.

Malva parviflora L.

Malvastrum corchorifolium (Desr.) Britton ex Small

Malvastrum coromandelianum (L.) Garcke

Malvella leprosa (Ort.) Krap.

Meximalva filipes (A. Gray) Fryx.
23481ZR

20035AM 20845RY 21998AM 23874AM

24286VA

22873VG

21401IT 21484IT 21661IT

$19873 \mathrm{GL}$

21487IT 21489IT 23415ZR

17601IT 19209GL

17771GL 18541GL 19872GL 21190IT

19242GL 23717AM 23876AM

17967IT 20544AM 20769RY

23614AM 23696AM

22781RA

20822RY 21457IT

19411GL 19543GL 20473GL

23773AM

23328ZR

17896AM

20784RY 23721AM 23867AM 24271SV 
Hinton \& Hinton: Hintons' Collections from South-Central Nuevo León and Adjacent Coahuila

Pavonia lasiopetala Scheele

Pavonia nepetaefolia (Standl.) Standl.

Sida abutifolia Mill.

Sida collina Schlecht.

Sida lindheimeri A. Gray

Sida rhombifolia $\mathrm{L}$.

Sida spinosa L.

Sphaeralcea angustifolia (Cav.) G. Don

Sphaeralcea hastulata A. Gray

Wissadula amplissima (L.) R. E. Fries
24437ST

$18881 \mathrm{GL} 23405 \mathrm{ZR}$

20370AM 20618DA 20760GL 22870VG

$19240 \mathrm{GL}$

20595AM

17750IT 24221MM

17684LN 20542AM 24222MM

17730GL 17843GL 19516GL 19570GL 24362ST

19426GL 19491GL 19629GL

17615IT 20575AM

\section{MARTYNIACEAE}

Martynia annua L.

20534AM

Proboscidea louisianica var. fragrans (Lindl.) Bretting

19981AM 20598GL 21555IT

\section{MENISPERMACEAE}

Cocculus carolinus (L.) DC.

Cocculus diversifolius DC.

17812ZR 24208MM

24287VA

MYRTACEAE

Psidium guajava L.

24167AN

\section{NYCTAGINACEAE}

Acleisanthes acutifolia Standl.

21958AM

Acleisanthes longiflora A. Gray

20793RY 22875VG

Acleisanthes obtusa (Choisy) Standl.

21533IT

Allionia incarnata L. var. incarnata

17524AM 19952AM 20783RY 21477IT

21526GL 21768AM 23133AM 23618AM

Boerhaavia anisophylla Torr.

22803AM 23995AM 24260SV 24481GL

Boerhaavia coccinea Mill.

Boerhaavia erecta L.

Boerhaavia linearifolia A. Gray

Cyphomeris crassifolia Standl.

17613IT 23722AM

17966IT

22040GL 24499GL

19066AM 20775RY 20860RY 21185IT

22703GL 23606AM 23690AM 23739AM

24498GL

Cyphomeris gypsophiloides (Mart. \& Gal.) Standl.

17658IT 21564GL 23117ZR

Mirabilis albida (Walt.) Heimerl

17317GL 19206GL 21122GL

Mirabilis glabrifolia (Ort.) I. M. Johnston

18498GL 18511GL 18572GL 18749GL

19011AM 19735GL 19753GL 19780GL

19908GL 20417AT 20487AT 23006AM

23033GL 23132ZR 23163AM 23587AM

23740AM 24403ST 
Mirabilis jalapa L.

Mirabilis linearis (Pursh) Heimerl.

Mirabilis longiflora L. var. Iongiflora

Mirabilis longiflora var. wrightiana (A. Gray) Kearney \& Peebles

Mirabilis multiflora (Torr.) A. Gray var. multiflora

Mirabilis nesomii B. L. Turner

Mirabilis oxybaphoides A. Gray

Mirabilis wrightiana A. Gray

Mirabilis sp.

Nyctaginea capitata Choisy
19922GL

$18757 \mathrm{GL}$

20491AT

23107AM

18436GL 18495GL $19621 \mathrm{GL}$

22319GL 22351AM

17556ZR 17926AT 18491GL 19702GL

$17603 I T$

17404ZR 24267SV

24282SH

\section{OLACACEAE}

Schoepfia schreberi Gmel.

$177491 \mathrm{~T}$

\section{OLEACEAE}

Forestiera angustifolia Torrey

Fraxinus cuspidata Torrey

Fraxinus greggii A. Gray var. greggii Menodora coulteri A. Gray var. coulteri Menodora coulteri var. minima Steyerm. Menodora hintoniorum B. L. Turner Menodora longiflora A. Gray Menodora muellerae Rehd.

\author{
18378GL 20102GL 22012AM \\ 17284GL 17650IT 18147IT 20196RY \\ 24370ST 24456GL \\ 18132GL 18689GL 21472IT \\ 20240AT 21048RA \\ 23042GL 24474GL \\ 19679GL TYPE \\ 20770RY 23851AM \\ 18220GL 18447GL 18820AT
}

\section{ONAGRACEAE}

Calylophus hartwegii (Benth.) Raven

Calylophus lavandulifolius (Torr. \& A. Gray) Rose Calylophus tubicula (A. Gray) Raven

Epilobium angustifolium L.

Gaura calcicola Raven \& Greg.

Gaura coccinea Pursh

Gaura drummondii (Spach) Torr. \& Gray

Gaura parviflora Dougl.

Jussiaea suffruticosa L.

Lopezia racemosa Cav.

Oenothera dissecta S. Wats.

Oenothera jamesii T. \& G.

Oenothera kunthiana (Spach) Munz

Oenothera macrosceles A. Gray
17765GL 18148GL 18370GL 18389GL 19523GL 19591GL 20467GL 20712AT 20758GL 21905GL 22317GL 23054AM 23623AM

18390GL 23035GL

20952GL

17869AT 18925AT

17772GL 20385AM 21164IT 22090GL 23997AM

17144GL 19416GL 20937AM 24395ST

21192IT

20252GL 20913AM

20535AM

19200GL 22448ZR 22537ZR

19506GL 19577GL 19590GL

$18776 \mathrm{GL}$

18733GL 22019GL

17814GL 21943AM 
Hinton \& Hinton: Hintons' Collections from South-Central Nuevo León and Adjacent Coahuila

Oenothera muelleri Munz

Oenothera primiveris A. Gray

Oenothera pubescens Willd. ex Spreng.

Oenothera rosea L'Hér. ex Ait.

Oenothera speciosa Nutt.

Oenothera stubbei Dietr. \& Raven

Oenothera tetraptera Cav.

Oenothera sp.

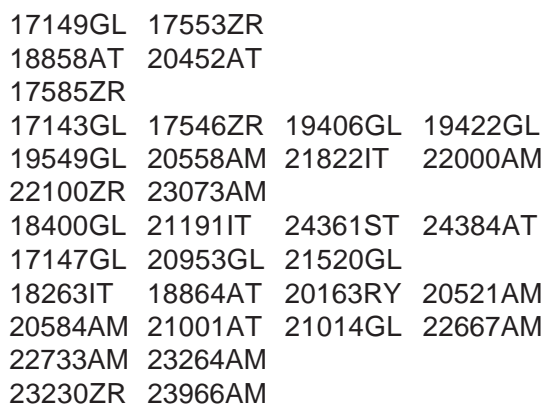

\section{OPHIOGLOSSACEAE}

Botrychium virginianum (L.) Swartz ssp. virginianum 22218GL 22306GL

\section{ORCHIDACEAE}

Bletia adenocarpa Reichb. f.

Bletia parkinsonii Hook.

Brachystele chiangii (M. C. Johnst.) Burns-Balough

Brachystele polyantha (Reichb. f.) Balough

Corallorhiza wisteriana S. W. Conrad

Corallorhiza sp.

Cranichis mexicana (A. Rich. \& Gal.) Schltr.

Dichromanthus cinnabarinus (Llave \& Lex.) Garay

Encyclia cyanocolumna (Ames, Hubb. \& Schweinf.) Dressler

Epipactis gigantea Dougl. ex Hook.

Galeottiella hintoniorum Todzia (ined.)

Goodyera oblongifolia Raf.

Goodyera striata Reichb. f.

Govenia liliacea (Llave \& Lex.) Lindl.

Govenia sp.

Habenaria guadalajarana S. Wats.

Hexalectris grandiflora (Rich. \& Gal.) L. O. Wms.

Malaxis brachyrrhynchos (Reichb. f.) Ames

Malaxis chica Todzia (ined.)

Malaxis corymbosa (S. Wats.) Kuntze

Malaxis ehrenbergii (Reichb. f.) Kuntze

Malaxis fastigata (Reichb. f.) Kuntze

Malaxis hintonii Todzia

Malaxis soulei L. O. Wms.

Malaxis tenuis (S. Wats.) Ames

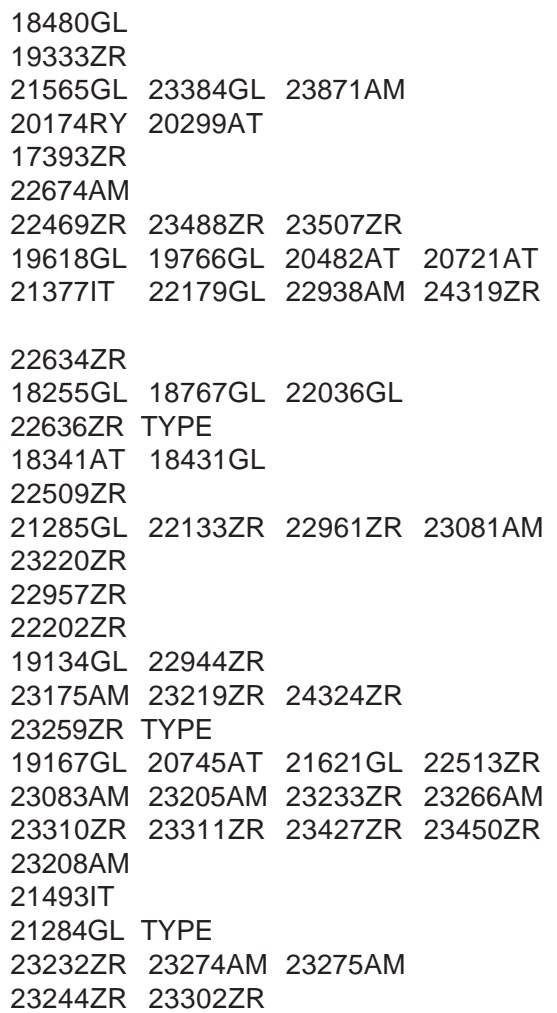


Ponthieva ephippium Reichb. f.

Ponthieva racemosa (Walt.) Mohr.

Prescottia tubulosa (Lindl.) L. O. Wms.

Sarcoglottis schaffneri (Rchb.f.) Ames

Schiedeella durangensis (Ames \&

Burns-Balough

Schiedeella eriophora (Robins. \& Greenm.) Schltr.

Schiedeella parasitica (A. Rich. \& Gal.) Schltr.

Spiranthes aurantiaca (Llave \& Lex.) Hemsl.

Spiranthes sp.
23222ZR

22540ZR

22689ZR

19260GL 21283GL 22450ZR 22536ZR

22811AM 22917AM 23487ZR

20289AT 21894GL 22778RA

18166GL 21830GL 22618ZR

18794GL 22826AM

17417ZR

19024AM

\title{
OROBANCHACEAE
}

Conopholis alpina Liebm. var. alpina

Conopholis alpina var. mexicana (A. Gray ex Wats.)

Haynes

Orobanche ludoviciana var. multiflora (Nutt.) Beck

Orobanche multicaulis Brandeg.
22614ZR 22918ZR

17583ZR 18798GL 20185RY

21789RY

19494GL

\section{OXALIDACEAE}

Oxalis albicans Kunth ssp. albicans

Oxalis albicans ssp. pilosa (Nutt.) Eiten

Oxalis corniculata L.

Oxalis decaphylla Kunth

Oxalis dillenii Jacq.

Oxalis drummondii A. Gray

Oxalis latifolia Kunth

Oxalis aff. latifolia Kunth

Oxalis madrensis S. Wats.

Brahea berlandieri Bartlett

\section{PALMAE}

19415GL 20939GL 22589ZR

21808GL 21823IT 24020AM

19910GL 20347AM

21033GL

$18283 \mathrm{GL}$

21049RA 21132GL 22106ZR 22240ZR

22810AM 22863GL 23646ZR 24507GL

17547ZR 18236GL 20256AT 20472GL

21002AT 22949ZR 23078AM 24241GL

22238ZR

22598LN

\author{
19984AM 21888GL 21889GL
}

\section{PAPAVERACEAE}

Argemone arida Rose

Argemone echinata Ownbey

Argemone mexicana L.

Argemone ochroleuca Sweet
19290RY 20130GL 20768RY

17676LN 17822RY 18502GL 19579GL 20397AM 20398AM 20942GL 20969AT 21509GL 21802GL

17794IT 18146IT 20357AM 21123GL 21188IT 21488IT 21844GL

17523AM 17555ZR 19099GL 20055AM 24007AM 
Hinton \& Hinton: Hintons' Collections from South-Central Nuevo León and Adjacent Coahuila

Argemone platyceras L. \& O.

Argemone subalpina McDonald

Argemone sp.

Bocconia latisepala Wats.

Hunnemannia fumariifolia Sweet

Hunnemannia hintoniorum Nesom

\author{
17549ZR 17759GL \\ 17383ZR 17550ZR \\ 19449GL 22706DA 23099AM 24394ST \\ 17945IT \\ 17450ZR 19276RY 19316ZR 20120RY \\ 21806GL 22759ZR 24140ST \\ 21876GL TYPE
}

PASSIFLORACEAE

Passiflora exsudans Zucc.

Passiflora foetida L.

17405ZR 22256ZR

17782IT 17947IT 21602GL

\section{PHYTOLACCACEAE}

Phytolacca icosandra L.

Phytolacca octandra L.

Rivina humilis $\mathrm{L}$.
17503ZR 24408ST

21615GL 24115ST

17617IT 19227GL 20798RY 21214IT

21412IT 21997AM 23877AM 24174AN

PINACEAE

Abies durangensis Martínez

Abies vejarii Martínez

Picea engelmannii var. mexicana Silba

Picea martinezii Patterson

Pinus cembroides Zucc.

Pinus culminicola Andresen \& Beaman

Pinus greggii Engelm.

Pinus hartwegii Lindl.

Pinus johannis M.-F. Robert

Pinus montezumae Lamb.

Pinus nelsonii Shaw

Pinus patula Schecht. \& Cham.

Pinus pinceana Gord.

Pinus pseudostrobus Lindl.

Pinus strobiformis Engelm.

Pinus teocote Schiede \& Deppe

Pseudotsuga menziesii (Mirb.) Franco

PIPERACEAE

Peperomia berlandieri Moq.

\author{
18363AT \\ 17274GL \\ 17972AT \\ 22672AM 23321ZR 24029AM \\ 17112GL 17492ZR 17768GL 18150GL \\ 17275GL 17920AT 20200AT 22423ZR \\ 17276GL 22071AT \\ 17277GL 17325ZR 17987AT 19068AT \\ 19394GL 19396GL \\ 19370AT \\ 22050GL 22051GL 22751ZR \\ 19108AM \\ 24025AM 24038AM \\ 21064RA \\ 21643GL 21644GL 21649GL \\ 17278GL 19395GL 19402GL \\ 18753GL 21629GL 21807GL 21903GL \\ 22052GL 22628ZR 22701AM 24026AM \\ 24032AM 24041AM 24042AM \\ 17270GL 17766GL 18154AT 18332AT \\ 18361AT 18935AT 19366AT 22980ZR
}

21302GL 21613GL 22138ZR 
Peperomia blanda Kunth Peperomia claytonioides Kunth

Piper auritum Kunth

\author{
17644ZR 17671IT 20854RY 23124ZR \\ 23077AM \\ 17828RY 23815ZR
}

\section{PLANTAGINACEAE}

Plantago australis ssp. hirtella (Kunth) Zahn

Plantago floccosa Dcne.

Plantago lanceolata L.

Plantago major L.

Plantago patagonica var. gnaphalioides (Nutt.) A. Gray

Plantago rhodosperma Dcne.
20334AM 20358AM 20359AM 22458ZR

24016AM

21273GL 21630GL

19407GL 24381AT

19439GL 21574GL 22089GL 23267AM

18438GL 18985GL

21866GL 21915GL 21934GL

PLATANACEAE

Platanus mexicana Moric.

Platanus occidentalis var. glabrata (Fern.) Sarg.

17591IT 17649ZR

Platanus sp. nov. Nixon \& Poole

22185SC

17514ZR 21543IT 22987ZR 24096AM

PLUMBAGINACEAE

Plumbago pulchella Boiss.

Plumbago scandens L.
19777GL 21121GL 21211IT 22599LN 17627IT 17725IT

\section{POACEAE}

Andropogon glomeratus (Walt.) B.S.P.

Andropogon virginicus $\mathrm{L}$.

Aristida divaricata Humb. \& Bonpl. ex Willd.

Aristida pansa Woot. \& Standl.

Blepharidachne bigelovii (S. Wats.) Hack.

Blepharoneuron tricholepis (Torr.) Nash

Bothriochloa barbinodis (Lag.) Herter

Bothriochloa aff. hybrida (Gould) Gould

Bothriochloa laguroides Herter ssp. torreyana

Bouteloua barbata Lag.

Bouteloua chasei Swallen

Bouteloua curtipendula (Michx.) Torrey

Bouteloua gracilis (Kunth) Lag. ex Griffiths

Bouteloua radicosa (Fourn.) Griffiths

Bouteloua repens (Kunth) Scribner \& Merrill

Bouteloua scorpioides Lag.

Bouteloua uniflora var. coahuilensis Gould \& Kapadia

Brachypodium pringlei Scribner ex Beal

Briza subaristata Lam.

Bromus carinatus Hook. \& Arn.
19963AM 22379GL

23290AM

$19746 \mathrm{GL}$

23010AM

23003AM

23968AM

19889GL 21503IT

20827RY

$19885 \mathrm{GL}$

23029GL

19644GL 20756GL 21532GL 21720GL

19681GL 20920AM 21070RA 21405IT

$19861 \mathrm{GL}$

21432 IT

$21379 I T$

$19740 \mathrm{GL}$

$19588 \mathrm{GL}$

21089RA 23143AM

21632GL

20284AT 22422ZR 23145AM 23158AM 
Hinton \& Hinton: Hintons' Collections from South-Central Nuevo León and Adjacent Coahuila

Buchloe dactyloides (Nutt.) Engelm.

Cenchrus incertus M. A. Curtis

Chloris submutica Kunth

Cortaderia selloana (Schult.) Asch. \& Graebner

Dactylis glomerata L.

Eragrostis intermedia A. Hitchc.

Eragrostis mexicana (Hornem.) Link ssp. mexicana

Erioneuron avenaceum (Kunth) Tateoka

Erioneuron grandiflorum (Vasey) Tateoka

Erioneuron pilosum (Buckl.) Nash

Erioneuron pulchellum (Kunth) Tateoka

Festuca amplissima Rupr. ex Fourn.

Festuca hintoniana E. Alexeev

Festuca pringlei St.-Ives

Heteropogon contortus (L.) P. Beauv. ex R. \& S.

Hordeum jubatum L.

Koeleria pyramidata (Lam.) P. Beauv.

Leptochloa dubia (Kunth) Nees

Lycurus phleoides Kunth

Melica montezumae Piper

Monanthochloe littoralis Engelm.

Muhlenbergia dubia Fourn.

Muhlenbergia emersleyi Vasey

Muhlenbergia gypsophila C. \& J. Reeder

Muhlenbergia macroura (Kunth) Hitchc.

Muhlenbergia parviglumis Vasey

Muhlenbergia rigida (Kunth) Trinius

Muhlenbergia robusta (Fourn.) Hitchc.

Muhlenbergia tenuifolia (Kunth) Trinius

Muhlenbergia villiflora Hitchc.

Oplismenus hirtellus (L.) P. Beauv.

Panicum acuminatum Swartz var. acuminatum

Panicum obtusum Kunth

Paspalum humboldtianum Flugge

Paspalum lividum Trinius

Paspalum pubiflorum Rupr. ex Fourn. var. pubiflorum

Phleum alpinum $\mathrm{L}$.

Poa annua $\mathrm{L}$.

Polypogon viridis (Gouan) Breistr.

Rhynchelytrum repens (Willd.) C. E. Hubbard

Scleropogon brevifolius Philippi

Setaria grisebachii Fourn.

Setaria leucopila (Scribner \& Merr.) K. Schum.

Setaria macrostachya Kunth

Setaria parviflora (Poir.) Kerguelen

Setaria pumila (Poir.) Schult.

Sorghum halepense (L.) Pers.

Sporobolus airoides (Torr.) Torr.

Sporobolus cryptandrus (Torr.) A. Gray

Sporobolus indicus (L.) R. Br.

Sporobolus wrightii Munro ex Scribner
18964AM 19418GL 22380GL

20612DA

19554GL

$24836 \mathrm{GL}$

$19131 \mathrm{GL}$

$21275 \mathrm{GL}$

19555GL 19572GL

23004AM

19617GL 19866GL

19739GL

19561GL 22331GL 23007AM

20278AT 21344GL 22302GL

17243GL TYPE

$19142 \mathrm{GL}$

24006AM

$19425 \mathrm{GL}$

20505AT

19556GL 19639GL

19863GL

22784RA

21982AM

21508GL

$21337 \mathrm{GL}$

19868GL 21531GL 23999AM

20506AT 20868AT

20835RY 20859RY

19887GL 20631DA

22553ZR

19793GL 19888GL

18789AM 19532GL

21204IT

$22081 \mathrm{GL}$

$19575 \mathrm{GL}$

20052AM

21952AM 23134GL

20910AM

$17295 \mathrm{GL}$

$17253 \mathrm{GL}$

21709GL 22084GL 22088GL

17623IT 19964AM 20020AM 23989AM

20757GL

17685LN 20851GL

20567AM

17520ZR 19774GL

20912AM

19886GL 19962AM

20911AM

$23031 \mathrm{GL}$

19571GL 22698AM

20908AM

19553GL 
Stipa clandestina Hackel

Stipa eminens Cav.

Stipa lobata Swallen

Stipa mucronata Kunth

Stipa multinodis Scribner ex Beal

Stipa aff. nelsonii Scribner

Stipa neomexicana (Thurber) Scribner

Stipa robusta (Vasey) Scribner

Stipa tenuissima Trinius

Tragus berteronianus Schult.

Tripsacum dactyloides (L.) L.

Trisetum spicatum (L.) Richt.

\author{
18214GL 19132GL 19408GL \\ 19742GL 19902GL \\ 20946GL \\ 17563ZR 20184RY \\ 20083GL \\ 20194RY \\ $19741 \mathrm{GL}$ \\ 19301GL 19361AT 20982AT \\ $19640 \mathrm{GL}$ \\ 21468IT \\ 20569AM \\ 17242GL 22431ZR
}

\section{POLEMONIACEAE}

Cobaea pringlei (House) Standl.

Gilia incisa Benth.

Gilia platyloba I. M. Johnston

Gilia rigidula var. acerosa A. Gray

Gilia rigidula Benth. var. rigidula

Gilia stewartii I. M. Johnston

Ipomopsis aggregata ssp. formosissima (Greene)

Wherry

Ipomopsis macombii (Torr.) Grant

Loeselia coerulea (Cav.) Don.

Loeselia greggii S. Wats.

Loeselia scariosa (Mart. \& Gal.) Walp.

Polemonium pauciflorum S. Wats.
22453ZR 23217ZR 23469ZR

17736IT 20331AM 20566AM 21021GL

21176IT 21885GL 23144AM 24173AN

24422ST

21561GL 21978AM

18832GL 19221GL 20621DA 21082RA

$19737 \mathrm{GL}$

18070GL 18187GL 18195GL 18510GL 22798GL

17191GL 18736GL 18919AT 20203AT

18179GL 18233GL 18745GL

17310GL 18595GL 19877GL 21754GL 19015AM

18602GL 18649GL 19187GL 19811GL 20079GL 20125GL 22026GL

17039GL 17876AT 18872AT 19393GL

POLYGALACEAE

Polygala alba Nutt.

Polygala aparinoides Hook. \& Arn.

Polygala barbeyana Chod.

Polygala dolichocarpa Blake

Polygala lindheimeri var. encosma (Blake) Wendt

Polygala lindheimeri A. Gray var. lindheimeri

Polygala macradenia A. Gray var. macradenia

Polygala oedophylla Blake
19090AM 19253GL 22375GL 24257SV 24433ST

22575ZR 22920AM 23298AM 24084AM 18707GL 18967AM 19733GL 20795RY 21062RA 22767RA

18655GL 23119ZR 23709AM 24003AM

$18227 \mathrm{GL} 18441 \mathrm{GL}$ 18944AM 21148SH

24240GL 24242GL

24264SV

17818GL 18135GL 18729GL 19282ZR

19308ZR 19340AM 19584GL 23859AM

24250SV 24477GL

18228GL 19291GL 20958GL 21862GL

21863GL 21976AM 22046GL 
Hinton \& Hinton: Hintons' Collections from South-Central Nuevo León and Adjacent Coahuila

Polygala subalata S. Wats.

Polygala turgida Rose

Polygala vergrandis W. Lewis

Polygala watsonii Chod.

\author{
17564ZR 20320AT 20724AT 21766AM \\ 21974AM 22694AM 23642ZR 23742AM \\ 23757AM 23863AM \\ 17856AM 18788AM 21949AM \\ 20126GL \\ 18941AM 19313ZR 21779RY 21874GL \\ 22656DA
}

\section{POLYGONACEAE}

Eriogonum atrorubens Engelm.

Eriogonum ciliatum Torrey

Eriogonum greggii Torr. \& A. Gray

Eriogonum jamesii var. undulatum (Benth.) Jones

Eriogonum punctatum Ell.

Polygonum aviculare L.

Polygonum densiflorum Meisn.

Polygonum hydropiperoides Michx.

Rumex crispus L.
17316GL 19002AM 19170GL 19830GL 20432AT

19728GL

22156SC 23624AM

17197GL 18267AT 18500GL 18930AT

20437AT 22401ZR 22801DA

23822AM

19551GL 22311GL

21518GL

19951AM

18549GL 20462GL

\section{POLYPODIACEAE}

Adiantum capillus-veneris $\mathrm{L}$.

Adiantum tricholepis Fée

Asplenium castaneum Schl. \& Cham.

Asplenium exiguum Bedd.

Asplenium monanthes $\mathrm{L}$.

Asplenium resiliens Kunze

Astrolepis beitelii (Mickel) Benh. \& Wind.

Astrolepis cochisensis (Goodd.) Benh. \& Wind.

Astrolepis crassifolia (Houlst. \& Moore) Benh. \& Wind.

Astrolepis integerrima (Hook.) Benh. \& Wind.

Astrolepis sinuata (Lag. \& Sw.) Benh. \& Wind.

Bommeria ehrenbergiana (Klotz.) Underw.

Campylonerum angustifolium (Sw.) Fée

Cheilanthes alabamensis (Buckl.) Kunze

Cheilanthes bonariensis (Willd.) Proctor
17323GL 17508ZR 18039AM 19302GL 21482IT

21043AM 22687ZR

$17189 \mathrm{GL}$

20142GL

17374ZR 19454GL 21293GL 22391ZR

22541ZR

19060AM 19367AT 19403GL 19709GL

20077GL 20865AT 20974AT 21030GL

21093RA 21292GL 22685ZR

23228ZR 23433ZR

17312GL 19645GL 19773GL

22259ZR

19093AM 21013GL 23401ZR 23731AM

17497ZR 19662GL 19760GL 20074GL

20960AT 23388GL

21217IT 22526ZR 23067AM

22653AM

17487ZR 20061AM 20580AM 20842RY

20915AM 21076RA 21675IT

17438ZR 19912GL 21573GL 21777RY

21837GL 22194ZR 22195ZR 22505ZR

23066AM 23420ZR 23467ZR 23658AM

23659AM 23726AM 
Acta Botánica Mexicana (1995), 30:41-112

Cheilanthes eatonii Baker

Cheilanthes hintoniorum Mend. \& Nesom

Cheilanthes hirsuta Link
Cheilanthes horridula Maxon
Cheilanthes kaulfussii Kunze
Cheilanthes leucopoda Link
Cheilanthes longipila Baker
Cheilanthes marginata Kunth
Cheilanthes meifolia Eaton
Cheilanthes myriophylla Desv.
Cheilanthes notholaenoides (Desv.) Maxon ex
Weatherby

Cheilanthes villosa Davenp. ex Maxon

Cheilanthes sp.

Cheiloplecton rigidum (Sw.) Fée

Cystopteris fragilis (L.) Bernh.

Dryopteris filix-mas (L.) Schott

Elaphoglossum pilosum (H. \& B. ex Willd.) Moore

Elaphoglossum sp.

Llavea cordifolia Lag.

Mildella fallax (Mart. \& Gal.) Nesom

Mildella intramarginalis (Kaulf. ex Link) Trev.

Notholaena aschenborniana Klotz.

Notholaena bryopoda Maxon

Notholaena candida (Mart. \& Gal.) Hook.

Notholaena incana Presl

Notholaena limitanea var. mexicana (Maxon) Broun Notholaena neglecta Maxon

Notholaena parvifolia Tryon

Notholaena schaffneri var. nealleyi (Seat.) Weatherby Notholaena standleyi Maxon

Notholaena sulfurea (Cav.) J. Smith

Pellaea atropurpurea (L.) Link.

Pellaea intermedia Mett. ex Kuhn

Pellaea aff. intermedia Mett. ex Kuhn

Pellaea notabilis Maxon
18704GL 19031AM 19049AM 20076GL 20855RY 20866AT 20975AT 21562GL 23649ZR

18639GL 18765GL TYPE 19315ZR

20157RY 21042AM 21513GL 22350AM 22695DA

23441ZR

20927AM 24259SV 24275SH

23712AM

20841RY 21182IT

20853RY 21018GL 22857RY

22087ZR 22121ZR 22153ZR

17513ZR 17708IT 22196ZR 22625AM $21111 \mathrm{GL}$

17324GL 17496ZR 18092GL 18385GL 18700GL 19042AM 19453GL 19768GL 20092GL

22608GL

23881AM

20579AM

17047GL 17304GL 17392ZR 17971AT

21290GL 21299GL 24024AM

22097ZR

22119ZR

22679AM

17486ZR 20057AM 20153GL 21289GL 22242ZR

22146ZR 23932AM

23635ZR

19759GL 21050RA 22607GL 23657AM

18083GL 19309ZR 19338AM 19651GL

$20071 \mathrm{GL}$

17489ZR 19974AM 20060AM 20165RY

20773RY 20856RY 21139SH 22593LN

21025GL 21144SH 21597GL 22199ZR

23260ZR

19048AM 19101AM 20965AT 20976AT

21058RA 22198ZR

19023AM 19724GL 20186RY 20925AM

20970AT 21505IT 22373GL

20924AM

19322AM

22257ZR

17755GL 17807ZR 18737GL 19467GL 20091GL 21306GL 21923GL

$18100 \mathrm{GL} 18299 \mathrm{GL} 18691 \mathrm{GL}$ 19094AM

19095AM 19687GL 20078GL 20863AT 20972AT

$21241 \mathrm{IT}$

20008AM 
Hinton \& Hinton: Hintons' Collections from South-Central Nuevo León and Adjacent Coahuila

Pellaea ovata (Desv.) Weatherby

Pellaea sagittata (Cav.) Link var. sagittata

Pellaea ternifolia (Cav.) Link

Pellaea sp.

Phanerophlebia umbonata Underw.

Polypodium erythrolepis Weath.

Polypodium fallacissimum Maxon

Polypodium guttatum Maxon

Polypodium hartwegianum Hook.

Polypodium plebejum Schl. \& Cham.

Polypodium plesiosorum Kunze

Polypodium polypodioides var. michauxianum Weath.

Polypodium subpetiolatum Hook.

Polypodium thyssanolepis A. Braun

Polystichum acrostichoides (Michx.) Schott

Polystichum hartwegii (Klotz.) Hieron.

Pteridium aquilinum var. pubescens Underw.

Pteris cretica L.

Pteris longifolia L.

Tectaria heracleifolia (Willd.) Underw.

Thelypteris ovata var. lindheimeri (C. Chr.) A. Smith

Thelypteris puberula (Baker) Morton

Woodsia mexicana Fée

Woodsia mollis (Kaulf.) J. Smith

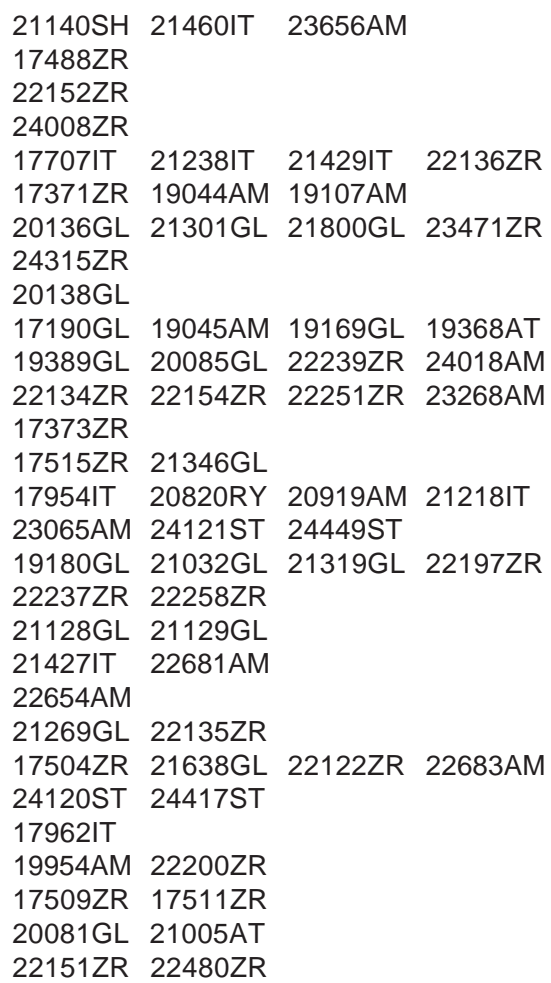

PORTULACACEAE

Portulaca oleracea L.

Portulaca pilosa L.

Talinopsis frutescens A. Gray

Talinum aurantiacum Engelm.

Talinum coahuilense (S. Wats.) P. Wilson

Talinum parviflorum Nutt.

\author{
17965IT 23017AM \\ 17661IT 19083AM 19663GL 20843RY \\ 21130GL 21160SH 21469IT 23016AM \\ 23165AM 23629AM 24432ST 24500GL \\ 20816RY \\ 18988AM 21098RA 24357ST \\ 20819RY \\ $18475 \mathrm{GL}$
}

PRIMULACEAE

Anagallis arvensis $\mathrm{L}$.

Androsace septentrionalis var. puberulenta (Rydb.) Knuth

Samolus cinerascens (Rob.) Pax \& Knuth

Samolus ebracteatus var. breviflorus Henrickson

Samolus parviflorus Knuth
17803ZR 20344AM

17915AT 18156AT 18822AT 20999AT 17855AM 19086AM 21945AM

18493GL 18604GL 18762GL 19939AM 20168RY 21480IT 21577GL 21847GL $18127 \mathrm{GL}$ 


\section{PYROLACEAE}

Chimaphila maculata (L.) Pursh Chimaphila umbellata (L.) W. Barton ssp. umbellata Monotropa hypopithys L.

Orthilia secunda (L.) House Pterospora andromedea Nutt.
17540ZR

17542ZR 22287GL 22929AM 23236ZR 18340AT 20442AT 20509AT 21624GL 22430ZR

18870AT 18936AT 19026AM 23972AM 20456AT 20510AT

RANUNCULACEAE

Aquilegia elegantula Greene Clematis drummondii Torr. \& A. Gray

Clematis pitcheri Torr. \& A. Gray

Delphinium madrense S. Wats.

Delphinium valens Standl.

Ranunculus peruvianus Pers.

\author{
17332ZR 18125GL 18152AT \\ 19552GL 20337AM 23601AM \\ 21922GL 22110ZR 22290GL 22459ZR \\ 22934ZR 23055AM 23250ZR 23258ZR \\ 22774RA 24217MM \\ 17418ZR 18883AT \\ 17004GL 17100GL 17350ZR 18854AT \\ 19324ZR 19390GL 20308AT 21255GL \\ 22221ZR 22833AM \\ 17372ZR 19325ZR 21300GL 21612GL \\ 24012AM \\ 20254AT 21303GL 22309GL \\ $18688 \mathrm{GL}$
}

Ranunculus petiolaris var. arsenei (Benson) T. Duncan 17863AM 21954AM

Ranunculus sierrae-orientalis (Benson) Nesom

\section{RESEDACEAE}

Reseda luteola L.

17881AT 19371AT

\section{RHAMNACEAE}

Ceanothus buxifolius Willd.

Ceanothus coeruleus Lag.

Ceanothus ferox Standl.

Ceanothus greggii A. Gray

Colubrina ehrenbergii Schlecht.

Colubrina greggii S. Wats.

Condalia ericoides (A. Gray) M. C. Johnston

Condalia fasciculata I. M. Johnston

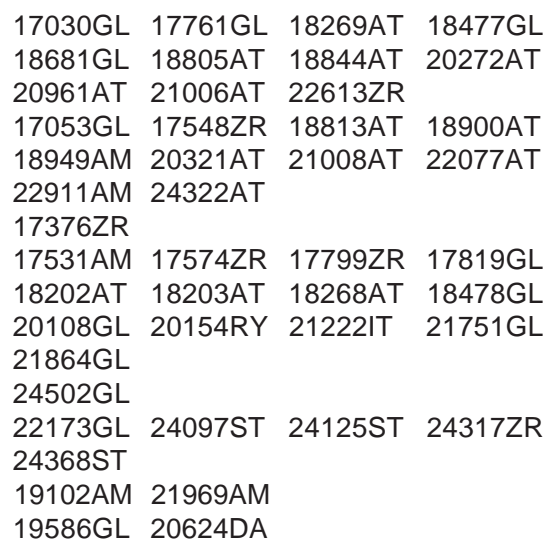

17030GL 17761GL 18269AT 18477GL 18681GL 18805AT 18844AT 20272AT 20961AT 21006AT 22613ZR 17053GL 17548ZR 18813AT 18900AT 18949AM 20321AT 21008AT 22077AT 22911AM 24322AT 17376ZR 17531AM 17574ZR 17799ZR 17819GL 18202AT 18203AT 18268AT 18478GL 20108GL 20154RY 21222IT 21751GL $21864 \mathrm{GL}$ $24502 G L$ 22173GL 24097ST 24125ST 24317ZR 24368ST

19102AM 21969AM 19586GL 20624DA 
Hinton \& Hinton: Hintons' Collections from South-Central Nuevo León and Adjacent Coahuila

Karwinskia humboldtiana (Roem. \& Schult.) Zucc.

Rhamnus betulifolia Greene

Rhamnus revoluta Rose

Rhamnus serrata Willd.

Rhamnus standleyana Wolf

Ziziphus Iloydii (Standl.) M. C. Johnston
19356GL 19896GL 20799RY 21052RA

21151SH 21199IT 21210IT 22808AM

22862GL 22868VG

18339AT 24388AT

$17801 Z R$

23226ZR

19218AM

21679IT

ROSACEAE

Alchemilla aphanoides L. f.

Alchemilla procumbens Rose

Amelanchier denticulata (Kunth) Koch

Amelanchier nervosa (Dcne.) Standl.

Amelanchier paniculata Rehd.

Amelanchier psilantha C. K. Schneid.

Cercocarpus fothergilloides Kunth

Cercocarpus macrophyllus Schneid.

Cercocarpus mexicanus Henrickson

Cercocarpus mojadensis Schneid.

Cercocarpus montanus Raf. var. montanus

Cercocarpus montanus var. paucidentatus (S. Wats.)

F. L. Martin

Cowania plicata D. Don

Crataegus baroussana Eggl.

Crataegus aff. greggiana Eggl.

Crataegus rosei Eggl.

Crataegus sp.

Duchesnea indica (Andrz.) Focke

Fragaria californica Cham. \& Schlecht.

Holodiscus discolor (Pursh) Maxim.

Holodiscus dumosus (Nutt.) Heller

Lindleya mespiloides Kunth

Petrophytum caespitosum (Nutt.) Rydb.

Physocarpus opulifolius var. intermedius (Rydb.)

B. Rob.

Potentilla leonina Standl.
23141AM 23156AM 23273AM

21620GL 22225ZR

17449DA 17665GL 19898GL 21022GL

21329GL 21746GL 23724AM

17499ZR

18376GL 18407GL 19350GL 22644ZR

23510ZR 24027AM

18786GL 19063AM 20150GL 21826GL

22724GL 24244GL 24505GL

18373GL 18388GL 18537GL 18739GL

18946AM 19399GL 20134GL 21827GL

21843GL 21990AM 22032GL 23769AM

24089AM 24164ST 24165ST

17339ZR 22283GL 22543ZR 22611ZR

22670AM 22690ZR

$18191 \mathrm{GL}$ TYPE

18193GL

24378AT

18115GL 18693GL 18702GL 19235GL

$17573 Z \mathrm{R}$ 17656IT 19660GL 19689GL

21988AM 22711DA 23626AM

18600GL 21254GL 22522ZR 23436ZR

21317GL 21376GL

18368GL 21322GL 21323GL 21442IT

21701IT

19114GL 19373GL 19388GL 24047GL

24136ST

17015GL 18163AT 18825AT 20981AT

22952ZR

17367ZR 22228ZR 22411ZR

17193GL 17916AT 18289AT 18889AT

17052GL 17343ZR 17796IT 17815GL

$18215 \mathrm{GL} 18489 \mathrm{GL} 18696 \mathrm{GL} 18701 \mathrm{GL}$

18982AM 19451GL 22045GL 24144ST

20459AT 20500AT 20864AT

22964ZR

17035GL 18843AT 20210AT 
Potentilla propinqua Rydb.

Potentilla rydbergiana Rose

Potentilla aff. rydbergiana Rose

Potentilla sp.

Prunus brachybotrya Zucc.

Prunus mexicana S. Wats.

Prunus serotina Ehrh. var. serotina

Prunus serotina var. virens (Woot. \& Standl.) McVaugh

Rosa carolina L.

Rosa serrulata Raf.

Rubus aboriginum Rydb.

Rubus arizonicus (Greene) Rydb.

Rubus caudatisepalus Calderón

Rubus flagellaris Willd.

Rubus idaeus var. sachilinensis (Levl.) Focke

Vauquelinia corymbosa ssp. angustifolia (Rydb.) Hess \& Henr.

Vauquelinia corymbosa ssp. latifolia (Standl.) Hess \& Henr.

Vauquelinia corymbosa var. heterodon (I. M. Johnston) Hess \& Henr.

\section{RUBIACEAE}

Bouvardia ternifolia (Cav.) Schlecht.

Chiococca alba (L.) Hitchc.

Chiococca pachyphylla Wernh.

Galium aparine L.

Galium dempsterae B. L. Turner

Galium juniperinum Standl.

Galium mexicanum Kunth

Galium microphyllum (A. Gray) Hemsl.

Galium rzedowskii Dempster

Galium uncinulatum DC.

Hedyotis intricata Fosb.

Hedyotis nigricans (Lam.) Fosb.

Hedyotis palmeri (A. Gray) W. H. Lewis
19071AT

17008GL 17329ZR 17354ZR 18366GL

18939AT

19369AT

18828AT

22746ZR

17282GL 18793GL 19387GL 20215GL

22563ZR 22741AM 22747ZR

17001GL 18714GL 21357GL 22668AM

22755ZR 24387AT

19151GL 21711GL

17576ZR

24087AM 24091AM

$17300 \mathrm{GL}$

22825AM

19152GL 22820AM 23324ZR 24078AM

24135ST

17983AT

19097GL 21989AM

19357GL 22704DA 23494ZR

$24455 G L$

18513GL 19598GL 20387AM 20836RY 22548ZR

$17728 I T$

$21559 I T$

$18710 \mathrm{GL}$

23110ZR

18731GL 18748GL 21587GL 24468GL

18290AT

19003AM 19469GL 20237AT 20438AT

20582AM 22274GL

17754GL 17797GL 18880GL 19033AM

19147GL 19710GL 20242GL 20262AT

21029GL 21330GL 21916GL 22208ZR

22414ZR 22895AM 22976ZR 23157AM

23176AM 23439ZR 24128ST 24410ST

22002AM

18278GL 18483GL 18517GL 18715GL

18744GL 18979AM 19690GL 19810GL

20007AM 20378AM 22017GL 22092GL

24351ST 24373ST

18218GL 18743GL 18993AM 20412AT

$22861 \mathrm{GL} 22871 \mathrm{VG} 24476 \mathrm{GL}$ 
Hinton \& Hinton: Hintons' Collections from South-Central Nuevo León and Adjacent Coahuila

Hedyotis rubra (Cav.) A. Gray

Randia aculeata L.

Randia rhagocarpa Standl.

Spermacoce confusa Rendle

Spermacoce laevis Lam.

Spermacoce tenuior $\mathrm{L}$.
18221GL 18451GL 19528GL 21933GL

17726IT 17931IT

17940IT

23430ZR 23519ZR

20324AM 20351AM

23297AM

\section{RUTACEAE}

Casimiroa pringlei (S. Wats.) Engler Choisya palmeri Standl.

Decatropis bicolor (Zucc.) Radlk.

Helietta parvifolia (A. Gray) Benth.

Ptelea trifoliata L.

Thamnosma texanum (A. Gray) Torr. var. texanum Zanthoxylum fagara (L.) Sarg.
18121IT 24059AM 24064AM

$18465 \mathrm{GL} 18705 \mathrm{GL} 21879 \mathrm{GL} 21897 \mathrm{GL}$

17673IT 20804RY 21540IT 22368GL

24346ST

17587IT 17941IT

17745IT 17792IT 18259IT 18416GL

19133GL 19384GL 21066RA 21084RA

21918GL 22720RA 23878AM

20124GL 21785RY

17742IT 21467IT 22732AM 22805AM

24070AM

\section{SALICACEAE}

Populus tremuloides Michx.

Salix nigra Marsh. var. nigra

Salix paradoxa Kunth

\author{
17976AT \\ 21924GL 24095AM \\ 18201AT 18207AT 18826AT 22754ZR
}

SAPINDACEAE

$\begin{array}{lll}\text { Cardiospermum corindum L. } & \text { 17614IT 20532AM 20588AM 21158SH } \\ & 21209 \mathrm{IT} \text { 21410IT 21448IT 24202MM } \\ & 24204 \mathrm{MM} & \\ \text { Cardiospermum dissectum Radkl. } & 24251 \mathrm{SV} & \\ \text { Dodonaea viscosa (L.) Jacq. } & 21824 \mathrm{IT} \text { 22761ZR } \\ \text { Sapindus saponaria var. drummondii (H. \& A.) Benson 17784IT 17957IT } & \\ \text { Sapindus saponaria L. var. saponaria } & 24203 \mathrm{MM} & \\ \text { Ungnadia speciosa Endl. } & 17519 \mathrm{ZR} \text { 17739IT } & \\ \text { Urvillea ulmacea Kunth } & 17604 \mathrm{IT} \text { 21420IT 24205MM }\end{array}$

SAURURACEAE

Anemopsis californica (Nutt.) H. \& A.

17861AM 21948AM

\section{SAXIFRAGACEAE}

Fendlera linearis Rehd.

21077RA 
Fendlerella lasiopetala Standl.

Fendlerella utahensis (Wats.) Heller

Heuchera amoena Rosendahl

Heuchera mexicana Schaffner

Heuchera rubescens Torrey

Heuchera sanguinea Engelm.

Philadelphus calcicolus $\mathrm{Hu}$

Philadelphus coulteri Wats.

Philadelphus maculatus (Hitchc.) $\mathrm{Hu}$

Philadelphus palmeri Rydb.

Ribes microphyllum Kunth

Ribes neglectum Rose
18171GL 18398GL 20295AT 21083RA

21588GL 24486GL

18216GL 18463GL 18719GL

17340ZR

22438ZR

17146GL 18952AM 19123GL 22974ZR

17912AT 18331AT 18855AT 20302AT

24093AM

17535ZR 18145IT

17584ZR 18712GL 19029AM 20445AT

17192GL

18819AT 22427ZR

17044GL 17336ZR 18810AT

\section{SCHIZAEACEAE}

Anemia mexicana Klotz.

17709IT 20058AM

\section{SCROPHULARIACEAE}

Agalinis gypsophila B. L. Turner Aureolaria greggii (Wats.) Pennell Bacopa procumbens (Mill.) Greenm. Calceolaria mexicana Benth.

Castilleja bella Standl.

Castilleja galehintoniae Nesom

Castilleja integrifolia L. f.

Castilleja lanata A. Gray

Castilleja mexicana (Hemsl.) A. Gray Castilleja rigida Eastw.

Castilleja scorzonerifolia Kunth

Castilleja sessiliflora Pursh

Castilleja tenuiflora Benth. var. tenuiflora

Castilleja tenuiflora var. xylorrhiza (Eastw.) Nesom

Escobedia laevis Schl. \& Cham.

Hemichaena spinulosa (S. Wats.) Thieret
18090GL 18328GL 18770GL 21501IT

18254GL 18462GL 19694GL 20609DA 17663IT

22745ZR 24092AM

17012GL 17049GL 17109GL 20998AT

18149GL 18422GL TYPE 18449GL

18530GL 20933AM 21914GL

17440ZR 17921AT 19020AM 20144GL

20743AT 21265GL 21986AM

18442GL 19443GL 19587GL 19605GL

20089GL 20251GL 21079RA 21155SH

22340GL 23032GL 23701AM

18448GL 19698GL 20119GL 24488GL

19688GL 20100GL 20301AT 20714AT

20726AT 20967AT 21063RA 22338GL 22339GL

17019GL 18235GL 19306ZR 20156RY

20273AT 20313AT 20386AM 20427AT

20778RY 21044AM 21341GL 21516GL

21726GL 21758AM 21767GL 21859GL

21929GL 22249ZR 22406ZR 23153AM

23317ZR 23621AM 24239GL

18258GL 19745GL

18574GL 20293AT 22409ZR

17791IT 17949IT 19193GL 19824GL 19894GL 21015GL 21061RA 21179IT 21725GL 24233MM 24372ST 24446ST 18037AM

19191GL 19204GL 
Hinton \& Hinton: Hintons' Collections from South-Central Nuevo León and Adjacent Coahuila

Lamourouxia dasyantha (C. \& S.) Ernst

Lamourouxia rhinanthifolia Kunth

Leucophyllum alejandrae Nesom

Leucophyllum candidum I. M. Johnston

Leucophyllum frutescens (Berl.) I. M. Johnston

Leucophyllum hintoniorum Nesom

Leucophyllum laevigatum var. griseum (I. M. Johnston) Henrickson

Leucophyllum laevigatum Standl. var. laevigatum

Leucophyllum pruinosum I. M. Johnston

Leucophyllum zygophyllum I. M. Johnston

Maurandya antirrhiniflora Kunth ex Willd.

Maurandya barclaiana Lindl.

Mecardonia vandellioides (Kunth) Penn.

Mimulus glabratus Kunth

Pedicularis canadensis L.

Penstemon amphorellae Crosswhite

Penstemon barbatus (Cav.) Roth

Penstemon campanulatus (Cav.) Willd. ssp. campanulatus

Penstemon galloensis Nesom

Penstemon havardii A. Gray

Penstemon lanceolatus Benth.

Penstemon leonensis Straw

Penstemon luteus Nesom

Seymeria scabra A. Gray

Seymeria tamaulipana B. L. Turner

Seymeria virgata (Kunth) Benth.

Sibthorpia repens (L.) Kuntze

Veronica persica L.

\author{
17452ZR 23609AM \\ 21298GL 21350GL 22129ZR 22927AM \\ 22321GL TYPE \\ 17640IT \\ 21476IT 23611AM 23616AM 24180LN \\ 20929AM TYPE 21961AM 21973AM \\ 22567ZR 23024AM 23128ZR 23131ZR \\ $23841 \mathrm{AM}$
}

18461GL 18504GL 19750GL 21060RA

22565ZR 23770AM 23890AM

17484ZR 21599GL 22335GL 23023AM 23600AM 23865AM

19211GL 20172RY 20603DA 23041GL

17184GL 22164GL 22260ZR 22349AM

22370GL 22468ZR 24490ST

17780IT 18479GL 20122GL 20377AM

17804ZR

17381ZR 19462GL 22211ZR 22743AM

22913AM

18473GL 19743GL 22358AM

17104GL 17357ZR 17870AT 17979AT

18878AT 20212AT 20285AT 20431AT

22432ZR 22975ZR 23164AM 24396ST

17055GL 17877AT 18565GL 18888AT

18898AT 20989AT 21012AT 21353GL

22126ZR 22281GL 23146AM

19166GL TYPE 21296GL 22107ZR

22215ZR 23916AM

18424GL 18496GL 18653GL

19726GL 20391AM 20413AT 21892GL

23118ZR 23171AM 23698AM

17385ZR 17579ZR 17879AT 20512AT

20744AT 22579ZR

21009AT TYPE

17653IT

17706IT 23753AM

18323GL 18742GL 19016AM 20419AT

$21331 \mathrm{GL} 23489 \mathrm{ZR}$

21699IT 22748ZR 23458ZR 23964AM

24088AM

18155AT 18927AT 22648AM

\section{SELAGINELLACEAE}

Selaginella gypsophila Smith \& Reeves

Selaginella lepidophylla (Hook. \& Grev.) Spring
18609GL 18633GL 18751GL 20160RY 19251GL 19277RY 19343AM 20175RY 20823RY 21143SH 
Selaginella novoleonensis Hieron.

Selaginella pallescens (Presl) Spring Selaginella parishii Underw.

Selaginella peruviana (Milde) Hieron.

Selaginella pilifera $\mathrm{A}$. $\mathrm{Br}$.

Selaginella wrightii Hieron.

Selaginella aff. wrightii Hieron.

\section{SIMAROUBACEAE}

Castela texana (T. \& G.) Rose

22710DA
24122ST

22532ZR 22638ZR 23126ZR 24448ST

21112GL

20938GL 23716AM

19104AM 19234GL 19265GL 19281RY

19335ZR 19975AM 20004AM 20059AM

20396AM 20571AM 20824RY 20852RY

20932AM 21041AM 21100RA 21138SH

21165IT 21202IT 21393IT

19755GL 19783GL 21141SH 21183IT

21403IT 21441IT 21783RY

20084GL

SOLANACEAE

Bouchetia erecta DC.

Capsicum annuum var. glabriusculum (Dunal) Heiser \& Pick.

Capsicum ciliatum (Kunth) Kuntze

Cestrum flavescens Greenm.

Cestrum laxum Benth.

Chamaesaracha coronopus (Dunal) A. Gray Chamaesaracha crenata Rydb.

Chamaesaracha sordida (Dunal) A. Gray

Datura inoxia Mill.

Datura quercifolia Kunth

Datura stramonium L.

Jaltomata procumbens (Cav.) J. Gentry

Lycianthes sp.

Lycianthes sp. nov. E. Dean

Lycium berlandieri Dunal

Margaranthus solanaceus Schlecht.

Nectouxia formosa Kunth

Nicotiana nudicaulis S. Wats.

Nicotiana repanda Willd.

Nicotiana trigonophylla Dunal

Petunia parviflora Juss.

Physalis chenopodiifolia Lam.

Physalis cinerascens (Dunal) Hitchc.

Physalis glutinosa var. exima (Standl.) Waterfall
18260IT 18467GL 18882GL 22018GL 23090AM 23148AM 24400ST

21542IT 24206MM

17588IT 17943IT 21500IT 23896AM

19331ZR 21295GL 21817IT 22623ZR

22677AM

17505ZR

19499GL 20477GL 20625DA

20774RY 23105AM

19654GL

17610IT 19272RY 21447IT

$19537 \mathrm{GL}$

21677IT

18522GL 22310GL 23161AM

20592AM 21019GL 22291GL

22222ZR

17850AM 18046GL 18099GL 19560GL

19603GL 20643DA

21440IT 23498ZR

17928AT

17724IT 19346GL 19376GL 20167RY

21842GL

21672IT 23774AM

17395DA 18512GL 19623GL 19856GL

21723GL

18603GL 24352ST

$17182 \mathrm{GL}$

20341AM 21171IT 21221IT 21449IT

17403ZR 
Hinton \& Hinton: Hintons' Collections from South-Central Nuevo León and Adjacent Coahuila

Physalis gracilis Miers

Physalis hederifolia A. Gray

Physalis macrophysa A. Gray

Physalis orizabae Dunal

Physalis philadelphica Lam.

Physalis sordida Fern.

Physalis virginiana var. sonorae (Torr.) Waterfall

Physalis viscosa var. cinerascens (A. Gray) Waterfall

Physalis walteri Nutt.

Physalis sp. nov. Martínez (\#1)

Physalis sp.

Solandra nitida Zucc.

Solanum douglasii Dunal

Solanum elaeagnifolium Cav.

Solanum erianthum D. Don

Solanum fendleri var. physaloides Correll

Solanum myriacanthum Dunal

Solanum nigrescens Mart. \& Gal.

Solanum aff. nigrescens Mart. \& Gal.

Solanum rostratum Dunal

Solanum tenuipes Bartlett

Solanum triquetrum Cav.

Solanum verrucosum Schlecht.

Witheringia mexicana (B. L. Rob.) Hunz.
23167AM

18978AM

21977AM 22345AM 22355AM

17347ZR

$17594 I T$

17930AT 18464GL 18558GL 21067RA $19502 \mathrm{GL}$

$18256 \mathrm{GL}$

20183RY

22104ZR TYPE 22255ZR 22832AM

22900AM 23229ZR

21314GL 22212GL 23335GL 23437ZR

23500ZR 24350ST

17802ZR 22931AM 22945ZR 23247ZR

23836AM

17607IT 20941GL 21125GL 21270GL

21381IT 21669IT 22227ZR 22286ZR

22352AM 22353AM 22659AM 23040GL

23277AM 23480ZR

19405GL 19440GL

17602IT 17752IT 21215IT 22527ZR

23651ZR 23830AM 24226MM

23504ZR

20001AM 21421IT 21551IT

18570GL 20557AM 20645DA 21479IT

$22301 \mathrm{GL}$

18074GL 19580GL

19659GL 22874VG 23021AM

20406AM 21115GL

18894AT 19703GL 20434AT 20786RY

21114GL 23314ZR 23326ZR

17690LN 17944IT 21239IT 21690IT

22893AM 24227MM 24450ST

STERCULIACEAE

Hermannia texana A. Gray

Melochia pyramidata L.

Melochia tomentosa L.

Waltheria indica L.

Taxus globosa Schl.

TILIACEAE

Tilia caroliniana Mill.

Triumfetta columnaris Hochr.
24290SV

17651IT 17790IT 21546IT 24223MM

24179LN

23727AM

\section{TAXACEAE}

17506ZR 22650AM

22147ZR

17410ZR 
TURNERACEAE

Turnera diffusa Willd.

19288RY 20797RY 22860RY 24253SV

TYPHACEAE

Typha domingensis Pers.

17902AM

ULMACEAE

Celtis laevigata Willd.

19358GL 24364ST

Celtis spinosa var. pallida (Torr.) M. C. Johnston 17775IT 21957AM 22172GL 24068AM

URTICACEAE

Laportea canadensis (L.) Wedd.

Parietaria decoris Mill.

Pilea microphylla (L.) Liebm.

Urtica chamaedryoides Pursh

Urtica spirealis Blume

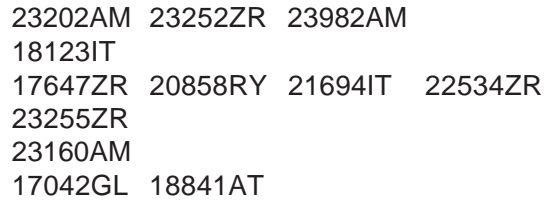

VALERIANACEAE

Valeriana albonervata B. L. Rob.

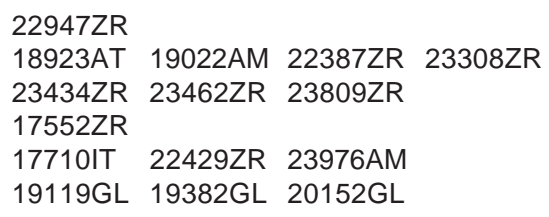

\section{VERBENACEAE}

Aloysia gratissima (Gill. \& Hook.) Tron.

Aloysia macrostachya (Torr.) Mold.

Aloysia triphylla (L'Hér.) Britt.

Aloysia wrightii (A. Gray) Heller

Bouchea prismatica var. brevirostra Grenz

Citharexylum berlandieri B. L. Rob.

Clerodendron bungei Steud.

Ghinia curassavica (L.) Millsp.

Glandularia bipinnatifida (Nutt.) Nutt.
18034GL 21103GL 21380IT 22369GL 22705DA

17482ZR 20782RY 20936AM 21054RA

21227IT 21791RY 23049DA 23615AM

23852AM 24066AM

22965ZR

$18596 \mathrm{GL}$

23062AM 23496ZR

17934IT

23517AM

21464IT

17254GL 19492GL 19545GL 20700GL 
Hinton \& Hinton: Hintons' Collections from South-Central Nuevo León and Adjacent Coahuila

Glandularia elegans (Kunth) Umber.

Glandularia polyantha Umber.

Glandularia rinconensis (Mold.) Umber.

Glandularia teucriifolia (Mart. \& Gal.) Umber.

Lantana achyranthifolia Desf.

Lantana camara L.

Lantana canescens Kunth

Lantana macropoda Torrey

Lantana velutina Mart. \& Gal.

Phyla nodiflora (L.) Greene

Phyla strigulosa (Mart. \& Gal.) Mold.

Priva lappulacea f. albiflora Mold.

Priva mexicana (L.) Pers.

Tetraclea coulteri A. Gray

Verbena brasiliensis Vell.

Verbena canescens Kunth

Verbena carolina L.

Verbena ehrenbergiana Schauer

Verbena halei Small

Verbena johnstonii (Mold.) Nesom

Verbena litoralis Kunth

Verbena neomexicana (A. Gray) Small var.

neomexicana

Verbena pumila Rydb.

Verbena recta Kunth

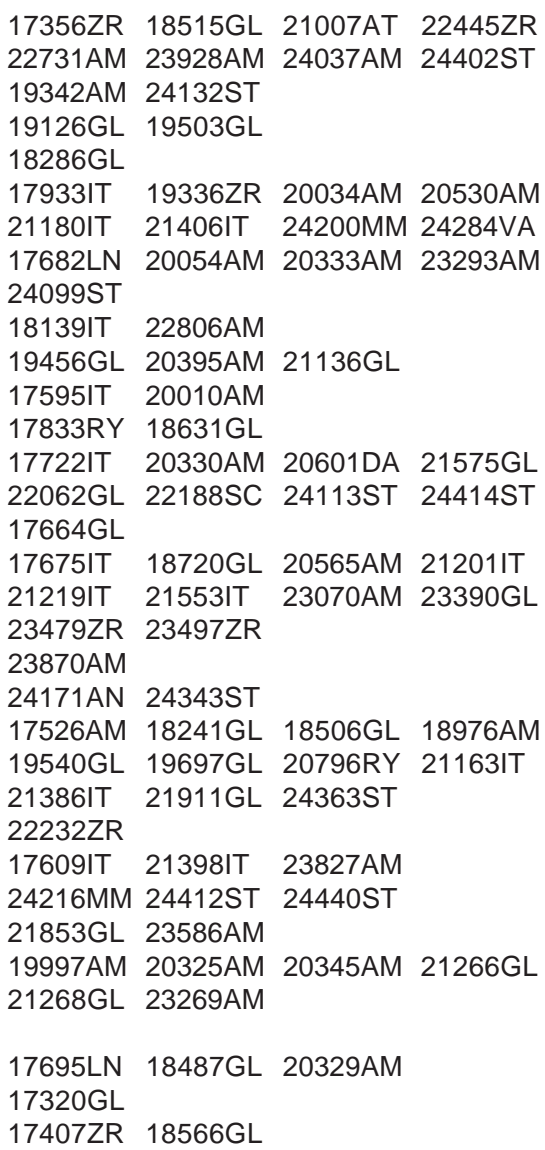

\section{VIOLACEAE}

Hybanthus verbenaceus (Kunth) Loes.

Viola flagelliformis Hemsl.

Viola galeanaensis M. S. Baker

Viola hookeri Kunth

VITACEAE

Parthenocissus quinquefolia (L.) Planch.

Vitis aff. berlandieri Planch.

Vitis cinerea Engelm.
20562AM 20590AM 21402IT 21452IT 21666IT 22029GL 22056GL 22971ZR

17102GL 17394ZR 18954AM 20312AT 22390ZR 22752ZR 22841AM 24081AM 22678AM

17723IT 21685IT

19213GL

19138GL 22824AM 24073AM 24141ST 
Acta Botánica Mexicana (1995), 30:41-112

ZYGOPHYLLACEAE

Kallstroemia parviflora Nort.

Larrea tridentata (DC.) Cov.

20591AM 21433IT 23038GL

Peganum mexicanum A. Gray 23993GL

Porliera angustifolia (Engelm.) A. Gray

$19429 \mathrm{GL}$

19279RY 